\title{
Qualität in der chirurgischen Therapie chronisch entzündlicher Er- krankungen der Nasennebenhöhlen
}

\section{Quality of Surgical Therapy of Chronic Inflammatory Diseases of the} Paranasal Sinuses

\section{(c) (i) ()ㅜ $\ominus$}

\section{Autoren}

Tanja Hildenbrand, Manuel Christoph Ketterer

Institut

Klinik für Hals-, Nasen- und Ohrenheilkunde,

Universitätsklinikum Freiburg

Schlüsselwörter

Endonasale endoskopische Nasennebenhöhlenchirurgie, Lebensqualität, Effizienz, Ergebnisqualität, Prozessqualität

Key words

Endonasal endoscopic surgery of the paranasal sinuses, multicentric data, Quality and efficiency in medicine,

process quality

Bibliografie

DOI https://doi.org/10.1055/a-1012-9383

Laryngo-Rhino-Otol 2020; 99: S5-S33

(c) Georg Thieme Verlag KG Stuttgart · New York

ISSN 0935-8943

Korrespondenzadresse

Dr. med. Tanja Hildenbrand

Univ. HNO-Klinik

Killianstr. 5

D-79106 Freiburg

tanja.hildenbrand@uniklinik-freiburg.de

\section{ZUSAMMENFASSUNG}

Die endonasale endoskopische Nasennebenhöhlenchirurgie hat sich als Standard der operativen Therapie bei chronisch entzündlichen Erkrankungen der Nasennebenhöhlen etabliert. Eine Verbesserung der subjektiven Beschwerden und objektiven Befunde konnte in zahlreichen Studien nachgewiesen werden. Aufgrund der häufig beobachteten Diskrepanz zwischen objektiven und subjektiven Ergebnissen nach Nasennebenhöhlenchirurgie kommt der Messung des sog. patient reported outcome eine zunehmende Bedeutung zu.
Qualität und Effizienz in der Medizin haben in den letzten Jahren zunehmend an Bedeutung gewonnen. In vielen Ländern und auch länderübergreifend wurden bereits durch Zusammenschluss von Experten, z.T unter Federführung verschiedener Fachgesellschaften, Initiativen zur Verbesserung der Qualität in der chirurgischen Versorgung gegründet.

Die vorliegende Arbeit stellt eine Übersicht qualitätsrelevanter Faktoren in der chirurgischen Therapie chronisch entzündlicher Erkrankungen der Nasennebenhöhlen anhand der vorhandenen Literatur, v.a. der Prozess- und Ergebnisqualität, dar. Ein besonderes Augenmerk liegt auf der subjektiven Bewertung der Ergebnisqualität durch den Patienten.

\section{ABSTRACT}

Endonasal endoscopic surgery of the paranasal sinuses is the gold standard in surgical treatment of chronic inflammatory diseases of the paranasal sinuses. Improvement of subjective complaints and objective findings has been confirmed in numerous studies. Due to the discrepancy between objective and subjective results after paranasal sinus surgery, the assessment of patient reported outcomes has gained importance. Quality and efficiency in medicine became increasingly important during the last years. In many countries and transnationally, the association of experts, partly coordinated by different medical societies, led to initiatives focusing on improvement of the quality of surgical care.

The present article represents an overview of quality-related factors in surgical treatment of chronic inflammatory diseases of the paranasal sinuses, summarizing the existing literature and focusing particularly on process and outcomes quality. Particular attention will be paid to the outcome quality individually assessed by the patients. 


\section{Inhaltsverzeichnis}

Zusammenfassung

S5

Abstract

1. Einleitung

2. Nasennebenhöhlenchirurgie in Deutschland

3. Chronische Rhinosinusitis

3.1 Präoperative Versorgung

3.1.1 Diagnose

3.1.2 Adäquate medikamentöse Therapie

3.1.3 Patientenselektion und patientenzentrierte Entscheidungsfindung

3.1.4 Zeitpunkt der Operation

3.1.5 Ambulant versus stationär

3.1.6 Chirurgische und radiologische Checklisten

3.2 Operative Versorgung

3.2.1 Endoskopische, mikroskopische und konventionelle Nasennebenhöhlenchirurgie

3.2.2 Navigation

3.2.3 Ausmaß der Operation/Radikalität

3.2.4 Ausbildung des Operateurs, Chirurgen-spezifische Faktoren

3.3 Postoperatives Ergebnis

3.3.1 Subjektive Beurteilung

3.3.1.1 Allgemeine Messinstrumente der gesundheitsbezogenen Lebensqualität

3.3.1.2 Spezifische Messinstrumente der gesundheitsbezogenen Lebensqualität

3.3.1.3 Produktivität

3.3.2 Objektive Beurteilung

3.3.2.1 Endoskopische Bewertung

3.3.2.2 Revisionsrate

3.3.2.3 Komplikationsrate

3.3.2.4 Riechtestung

3.3.3 Core Outcome Sets (COS)

3.3.4 Effizienz

4. Rezidivierende akute Rhinosinusitis

4.1 Diagnose

4.2 Medikamentöse Therapie

4.3 Patientenselektion und patientenzentrierte Entscheidungsfindung

4.4 Postoperatives Ergebnis

4.4.1 Allgemeine Messinstrumente der gesundheitsbezogenen Lebensqualität

4.4.2 Spezifische Messinstrumente der gesundheitsbezogenen Lebensqualität

4.4.3 Produktivität

Danksagung

Literatur

\section{Einleitung}

Die chronische Rhinosinusitis (CRS) ist eine häufige Erkrankung mit signifikanten Auswirkungen auf die Lebensqualität, die direkten und indirekten Kosten für das Gesundheitssystem und die Produktivität [1]. Die Auswirkungen der CRS auf die allgemeine Lebensqualität sind in den Bereichen körperliche Schmerzen und soziale Funktionsfähigkeit größer als bspw. bei der Angina pectoris, COPD und kongestiven Herzinsuffizienz [2]. Die Prävalenz der CRS in Europa wird mit 10,9\% (95\% Cl: 6,9-27,1\%) angegeben [3]. In den USA ist die Rhinosinusitis der sechsthäufigste Grund einer ambulanten ärztlichen Vorstellung und die häufigste Ursache für die Verschreibung von Antibiotika [4].

Die American Rhinologic Society führte eine Studie durch, mit dem Ziel, rhinologische Erkrankungen anhand epidemiologischer, klinischer und ökonomischer Daten hinsichtlich der Notwendigkeit der Qualitätsverbesserung zu priorisieren. Die CRS und die rezidivierende akute Rhinosinusitis wurden zu 2 der 3 wichtigsten Erkrankungen bestimmt [5]. Bemühungen zur Qualitätsverbesserung im Rahmen chirurgischer Eingriffe sollten darauf abzielen, den richtigen Eingriff zum richtigen Zeitpunkt bei dem richtigen Patienten durchzuführen. Die nicht sachgemäße Nutzung von Gesundheitsleistungen, d.h. sowohl übermäßige als auch zu geringe Formen der Nutzung, sollen verhindert werden. Trotz nationaler und internationaler evidenz-basierter Leitlinien, wie der deutschen S2k-Leitlinie Rhinosinusitis [6], existieren mitunter große Schwankungen in der Anwendung dieser Leitlinien in der klinischen Praxis. Zudem zeigen sich international große geografische Unterschiede in der Häufigkeit und dem Ausmaß von Nasennebenhöhleneingriffen [7-10].

Die Therapieoptionen der CRS sind physikalisch (z.B. Nasenspülungen), medikamentös und chirurgisch. Die primäre Therapieform ist die medikamentöse Therapie. Die Indikationen zur chirurgischen Therapie sind eine fehlende ausreichende Beschwerdebesserung trotz adäquater medikamentöser Therapie, sowie drohende oder manifeste Komplikationen der Entzündung. Ist ein konservativer Behandlungsversuch von vorneherein wenig aussichtsreich, nicht möglich oder nicht erwünscht, kann die Therapie auch unmittelbar chirurgisch erfolgen [6]. Die chirurgische Standardtherapie ist die endonasale endoskopische Operation $[6,11,12]$. Nach der Gesundheitsberichterstattung des Bundes wurden im Jahr 2017 in Deutschland 455399 Operationen an der Nase und den Nasennebenhöhlen vollstationär durchgeführt. Die endonasale endoskopische Nasennebenhöhlenchirurgie hat sich in mehreren prospektiven Studien und systematischen Reviews als effektive Therapie der chronischen Sinusitis erwiesen [11, 13-19]. Sie kann, in Kombination mit einer medikamentösen Therapie, die kurz- und langfristige Lebensqualität und Beschwerden durch Begleiterkrankungen, wie Asthma und Schlafstörungen, verbessern und scheint kosteneffektiver als eine alleinige medikamentöse Therapie zu sein [20-25] (s.u.).

Qualität im Gesundheitswesen kann nach Donabedian in 3 untereinander verbundenen Dimensionen beschrieben werden [26]:

- Strukturqualität: hierbei handelt es sich um die allgemeinen Rahmenbedingungen und Voraussetzungen, wie z.B. bauliche und apparative Ausstattung, Anzahl/Qualifikation der Mitarbeiter, organisatorischer Aufbau der behandelnden Einrichtung

- Prozessqualität: dies beschreibt die Qualität der Leistungserbringung und somit der Behandlung. Hierzu zählen die Einhaltung fachlicher Standards und Beachtung von Leitlinien. 
- Ergebnisqualität: hierbei geht es um die Rückmeldung über die Ergebnisse der Behandlung, so z. B. die Patienten- oder Mitarbeiterzufriedenheit, Richtigkeit der Diagnose, Änderung des Gesundheitszustandes (Symptome, Lebensqualität). Die Messung der Ergebnisqualität in der Medizin gestaltet sich häufig schwierig, da die Verbesserung des Gesundheitszustandes des Patienten nicht ohne weiteres objektivierbar und messbar ist. Hier kommt dem sog. „patient reported outcome“ eine wesentliche Bedeutung zu, da die Kunden- bzw. Patientenorientierung einen wesentlichen Grundsatz des Qualitätsmanagements darstellt.

Das vorliegende Referat beruht auf einer systematischen Literaturrecherche in PubMed und Medline sowie der Cochrane Library. Deutsch- und englischsprachige Artikel wurden, aufgrund des Zeitpunkts der Erstellung des Referates, bis Juni 2019 berücksichtigt. Es kann kein Anspruch auf Vollständigkeit erhoben werden. Da die akute Rhinosinusitis in der Regel, d.h. wenn keine Komplikationen auftreten, konservativ behandelt wird, beschränkt sich die vorliegende Übersicht auf die chronischen Rhinosinusitis mit ohne ohne Polyposis nasi (CRSsNP, CRSwNP) und die rezidivierende akute Rhinosinusitis (RARS). Aufgrund der Seltenheit und der wenigen Studien für Patienten im Kindesalter werden die Ergebnisse für die erwachsene Bevölkerung dargestellt.

\section{Nasennebenhöhlenchirurgie in Deutschland}

Ein wesentlicher Grundsatz des Qualitätsmanagements ist die kontinuierliche Verbesserung, die sich im PDCA-Zyklus (Plan, Do, Check, Act) widerspiegelt. Um die Notwendigkeit von Verbesserungen beurteilen zu können, ist es zunächst notwendig, den aktuellen Zustand zu erfassen.

Um den aktuellen Stand der Nasennebenhöhlenchirurgie in Deutschland zu beurteilen, führten wir eine Umfrage an allen HNOKliniken durch. Ein anonymisierter Fragebogen mit 10 Fragen zu OPZahlen, OP-Technik, technischer Ausstattung, interdisziplinärer Zusammenarbeit, Nachsorge, Ausbildung und Lebensqualität wurde an alle Ordinarien und Chefärzte der HNO-Kliniken in Deutschland versandt.

Insgesamt wurden 171 Fragebögen an 37 HNO-Universitätskliniken und 134 städtische, private und kirchliche HNO-Kliniken versandt. Es wurden 136 Fragebögen beantwortet (35 von Universitätskliniken, 96 von städtischen, privaten und kirchlichen HNO-Kliniken, 5 ohne Angaben). Die Rücklaufquote betrug 79,5\%.

$51,9 \%$ der Kliniken führen pro Jahr zwischen 200 und 400 Operationen, 37,2\% über 400 Eingriffe bei benignen Erkrankungen der Nasennebenhöhlen durch ( $\triangleright$ Abb. 1). Die Zahl der Operationen bei malignen Erkrankungen ist deutlich geringer: 59,6\% der Kliniken füh-

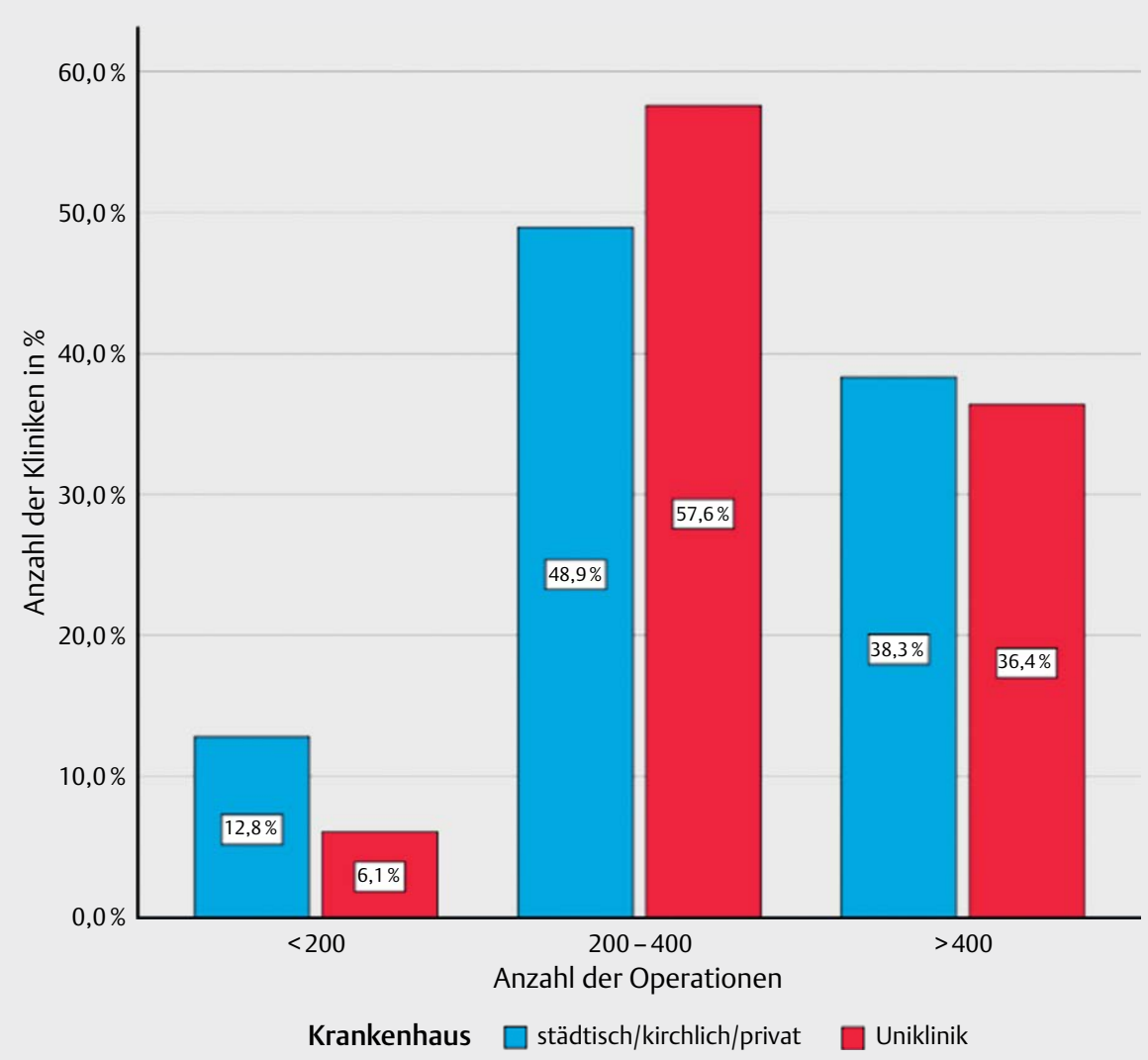

- Abb. 1 Anzahl der Operationen bei benignen Erkrankungen der Nasennebenhöhlen. 


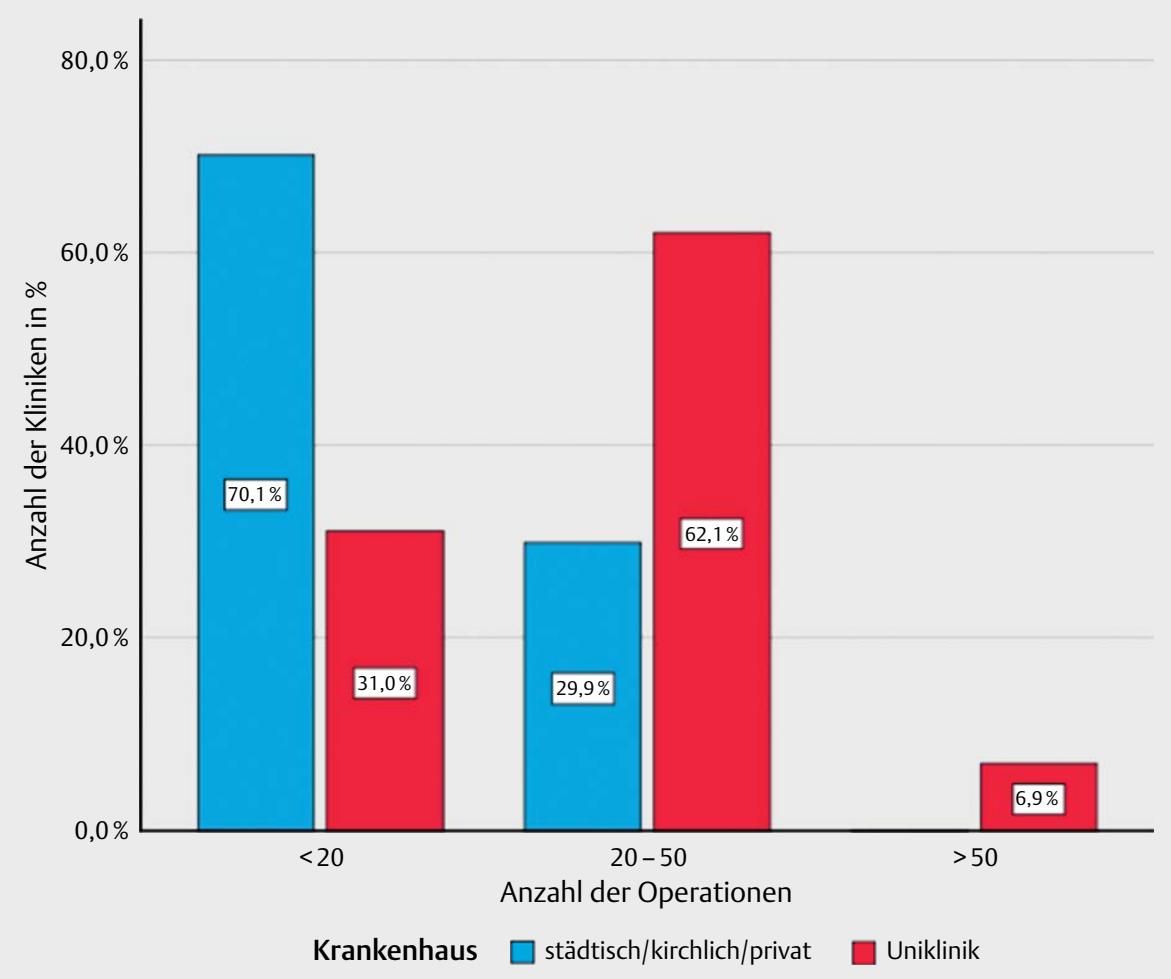

- Abb. 2 Anzahl der Operationen bei malignen Erkrankungen.

ren $<20$ Operationen pro Jahr durch. Zwischen 20 und 50 Operationen werden von 38,4\% der Kliniken durchgeführt und mehr als 50 Operationen geben lediglich $2 \%$ der Kliniken an ( $\triangleright$ Abb. 2).

$83,1 \%$ aller Kliniken gaben an, an einem interdisziplinären Tumorboard teilzunehmen und 68,4\% führen interdisziplinäre Tumoroperationen bei Erkrankungen der Nasennebenhöhlen und Schädelbasis durch. Nur ein Teil der Kliniken, die Patienten in einem interdisziplinären Tumorboard besprechen, führt auch interdisziplinäre Operationen durch (Unikliniken: 77,1\%, städtische/kirchliche/private Kliniken 55,8\%). Zum Teil wurden hier, wenn auch nicht explizit erfragt, schlechte Erfahrungen während vergangener Kooperationen als Grund angegeben, weshalb die Operationen i.d.R. zweizeitig erfolgen.

Die meisten Kliniken verfügen über ein Endoskopiesystem und führen die Operationen entweder rein endoskopisch (49,6\%) oder endoskopisch/mikroskopisch kombiniert (48,1\%) durch (• Abb. 3). Ein HD-Video-System für die endoskopische Nasennebenhöhlenchirurgie besitzen 88,1 \% der Kliniken. Ähnlich verbreitet ist die Ausstattung mit einem Navigationssystem. Alle Universitätskliniken besitzen ein Navigationssystem, welches $80 \%$ der Kliniken mehr als $1 \mathrm{x}$ pro Monat einsetzen. 79,2\% der städtischen, kirchlichen und privaten Kliniken besitzen ein solches System, das in 62,5\% mehr als $1 \mathrm{x}$ pro Monat genutzt wird.

Die Ausbildung der Assistenzärzte in der Nasennebenhöhlenchirurgie beginnt in den meisten Kliniken zwischen dem 2. und 4. Ausbildungsjahr (2.-3. Jahr: 48,1\%, 3.-4. Jahr 38,2\%). In fast allen Kliniken besuchen Assistenzärztinnen und -ärzte im Rahmen der Ausbildung einen Operationskurs zur Nasennebenhöhlenchirurgie (96,9\%).
Eine Präparation an Körperspendern vor Ort ist nur in 28 Kliniken möglich (12 städtische/kirchliche/private Kliniken, 16 Unikliniken).

Eine CT-Checkliste zur präoperativen systematischen Analyse von CT-Bildern wird von 63,2\% der Kliniken genutzt.

Die postoperative Nachsorge wird in den Universitätskliniken seltener $(24,42 \%)$ durch den Operateur oder andere Mitarbeiter der Klinik durchgeführt als in städtischen/kirchlichen/privaten Kliniken (55,72\%). Häufiger werden die Patienten durch die niedergelassenen Kollegen weiter betreut ( $\vee$ Abb. 4).

Die Lebensqualität, als eine Variable zur Beurteilung des Ergebnisses nach einer Operation der Nasennebenhöhlen, wird standardmäßig nur in 7,6\% der Kliniken bestimmt, in einigen Fällen erfolgt dies ausschließlich im Rahmen von Studien.

\section{Chronische Rhinosinusitis}

\subsection{Präoperative Versorgung}

Auch wenn es sich bei dem Thema des Referates um die Qualität in der chirurgischen Therapie der CRS handelt, ist es notwendig, einzelne Phasen der präoperativen Versorgung miteinzubeziehen, da bereits hier wichtige Weichen für eine qualitativ hochwertige chirurgische Versorgung gestellt werden. Das Quality Improvement Commitee der American Rhinologic Society hat vier qualitätsrelevante Bereiche der präoperativen Versorgung identifiziert [27]. Dies sind Diagnose, adäquate medikamentöse Therapie, patientenzentrierte Diskussion von Behandlungsoptionen und Selektion zur chirurgischen Intervention. 


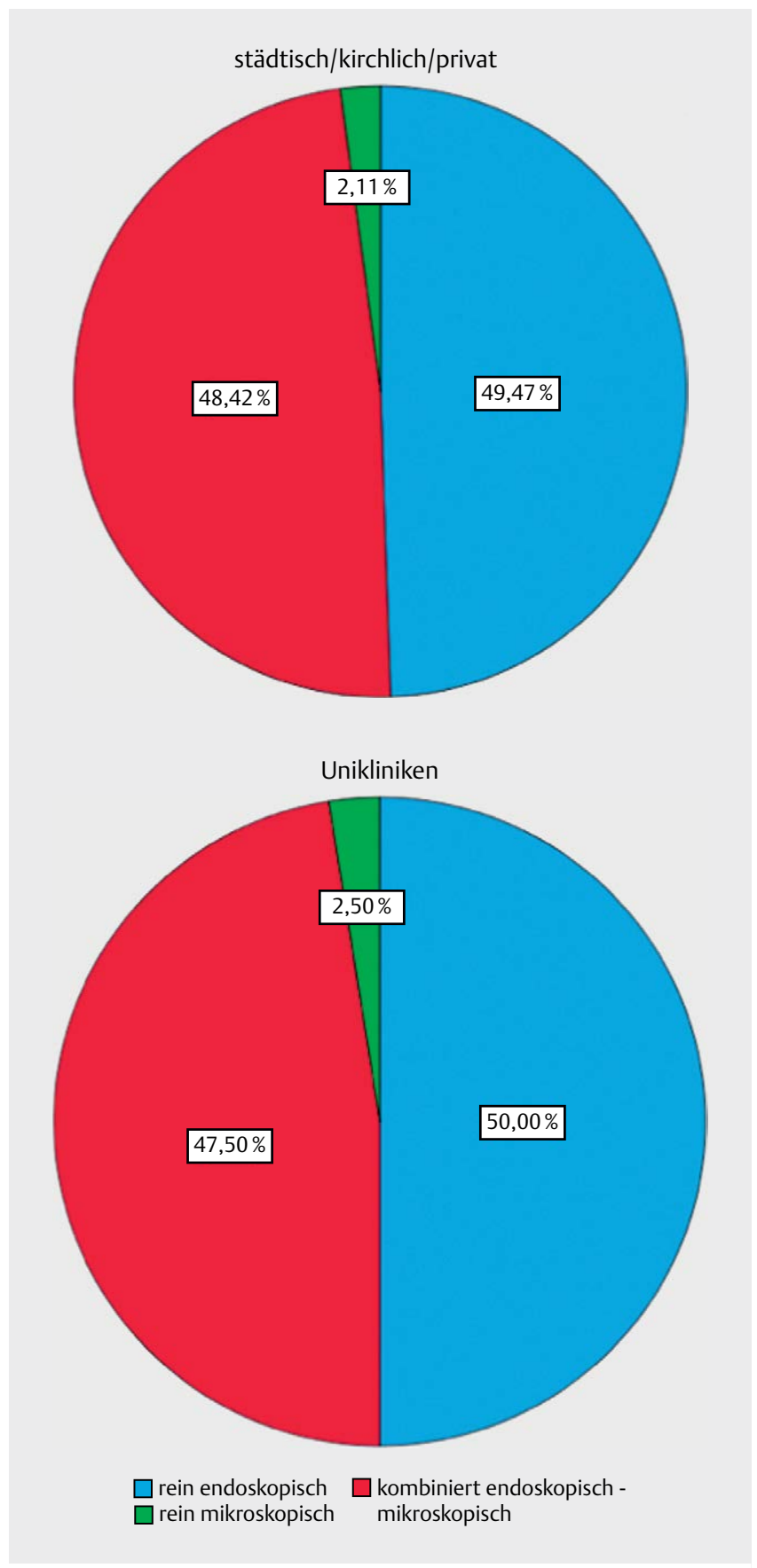

- Abb. 3 Technische Durchführung der Operationen.

Ein wichtiges Element des Qualitätsmanagements sind Qualitätsindikatoren und Kennzahlen. Sie sollen die faktengestützte Entscheidungsfindung ermöglichen und Abläufe nachvollziehbar und bewertbar machen. Im Gegensatz zur Industrie ist die Definition und Erhebung dieser Kennzahlen in der Medizin häufig schwierig. Bisher gibt es keine allgemein akzeptierten Qualitätsindikatoren spezifisch für die Nasennebenhöhlenchirurgie. In einem Leitlinien- und Konsensus-basierten Ansatz einer Expertengruppe wurden neun Qualitätsindikatoren für die CRS entwickelt ( $\triangleright$ Tab. 1) [28]. Die Gruppe betont, dass diese Qualitätsindikatoren abhängig von geografischen Unterschieden sowie Unterschieden in Ressourcen und Patienten- gut entsprechend angepasst werden müssen. Bei den identifizierten Qualitätsindikatoren handelt es sich ausschließlich um Indikatoren der Prozessqualität. Das Fehlen von Indikatoren der Struktur- und Ergebnisqualität wird auf den Fokus von Leitlinien auf die klinische Praxis und nicht die Systemstruktur und -leistung zurückgeführt. Diese Qualitätsindikatoren wurden bisher nicht auf ihre Variabilität, Validität und Fähigkeit, die Patientenversorgung zu verbessern, geprüft. Es bleibt auch unklar, wie sie in großem Maßstab in der klinischen Praxis gemessen und dokumentiert werden sollen, da dies die Voraussetzung für Qualitätsverbesserungsmaßnahmen darstellt.

\subsubsection{Diagnose}

Die diagnostischen Kriterien der CRS nach dem European Position Paper on Rhinosinusitis and Nasal Polyps (EPOS) sind weitestgehend akzeptiert. Sie finden sich auch in der deutschen S2k-Leitlinie Rhinosinusitis. Die Diagnose wird gestellt, wenn mindestens 2 der folgenden Symptome für mindestens 12 Wochen bestehen:

- Nasenatmungsbehinderung und/oder Rhinorrhoe

- +/-Gesichtsschmerz/Druck

- +/-Hyposmie

und wenn gleichzeitig objektivierbare klinische Zeichen, wie endoskopische Veränderungen (Sekret/Ödem im mittleren Nasengang, Polyposis nasi) oder typische Zeichen einer CRS im CT sichtbar sind [11]. Entsprechende Kriterien finden sich auch in den Leitlinien der American Academy of Otolaryngology - Head and Neck Surgery und im International Consensus Statement on Allergy and Rhinology Rhinosinusitis (ICAR) [29, 30]. Die Kombination aus klinischen Symptomen und objektiven Kriterien erhöht die diagnostische Genauigkeit und Spezifität [31].

\subsubsection{Adäquate medikamentöse Therapie}

Eine Diskussion aller möglichen Ansätze einer präoperativen medikamentösen Therapie einer CRS würde den Rahmen dieses Referates sprengen. Es wird hier auf die entsprechenden deutschen und internationalen Leitlinien verwiesen $[6,11]$. Problematisch ist, dass kein allgemein akzeptierter Standard existiert und die Evidenz zum Teil dürftig ist. Für eine präzisere Stratifizierung der Erkrankung und Standardisierung der Therapie ist ein besseres Verständnis der Pathogenese der CRS erforderlich. Die beste Evidenz existiert für topische Steroide durch eine große Anzahl randomisierter placebo-kontrollierter Studien mit Verbesserung subjektiver Symptome, der Polypengröße und der objektiven Messung des nasalen Atemstroms [32]. Ein Artikel zur Qualität der präoperativen Versorgung sieht die beste Evidenz für salzhaltige Nasenspülungen und topische Steroide [27]. Er legt beide Therapien als Minimum eines konservativen Therapieversuches fest, bevor eine chirurgische Intervention in Erwägung gezogen werden sollte. Diese Empfehlung geht aus einem internationalen Konsensusverfahren nach der RAND/UCLA-Methode hervor [33]. Bei dieser Methode, die in den 80er Jahren in den USA entwickelt wurde, werden Kriterien auf Grundlage der besten verfügbaren Evidenz in Kombination mit der gemeinsamen Bewertung von Experten entwickelt. Sie kann angewandt werden, wenn evidenzbasierte Leilinien auf Grundlage randomisierter kontrollierter Studien nicht möglich sind. Zusätzlich werden systemische Steroide als eine Therapieoption bei der chronischen Rhinosinusitis mit Polyposis nasi (CRSwNP) und systemische Antibiotika bei der chronischen 


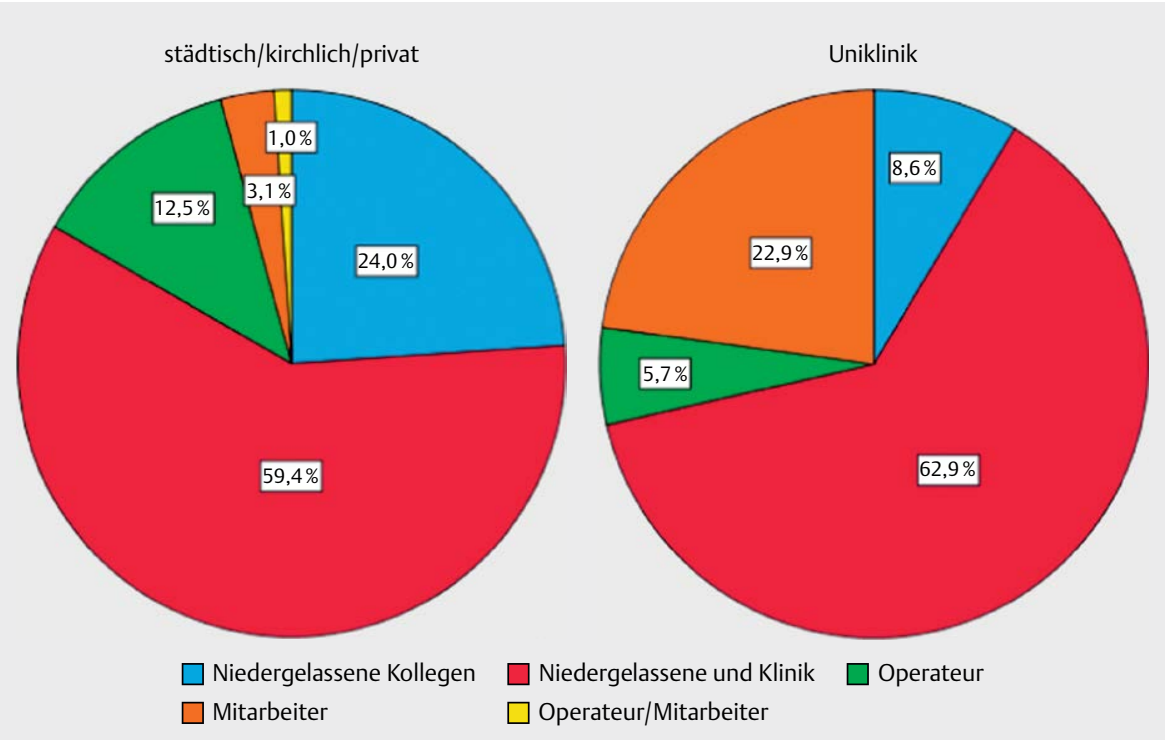

- Abb. 4 Postoperative Nachsorge der Patienten nach Nasenneben-höhleneingriffen.

- Tab. 1 Qualitätsindikatoren für die Diagnose und Behandlung von Patienten mit CRS nach Cottrell [28].

Diagnose der CRS aufgrund von klinischen Symptomen und mindestens 1 objektiven Befund in der Endoskopie oder im CT

Unterscheidung zwischen CRSwNP und CRSsNP

Die Bildgebung der Wahl für die CRS ist das CT

Initiale Therapie der CRSwNP mit topischen Steroiden und kurzzeitig systemischen Steroiden

Keine Verordnung von topischen oder systemischen Antimykotika

Unterstützende Therapie mit Salzspülungen zeigt evidenzbasierte Wirksamkeit. Andere unterstützende Therapien haben nur eine eingeschränkte Evidenzlage, die den Einsatz rechtfertigt

Nasennebenhöhlenchirurgie kann für Patienten mit persistierender Symptomatik nach adäquater medikamentöser Therapie indiziert sein

Die Weiterführung der medikamentösen Therapie postoperativ ist wichtig für den Erfolg der Behandlung und sollte bei allen Patienten in Betracht gezogen werden

Intravenöse und topische Antibiotika sollten für Routinefälle nicht angewandt werden

Rhinosinusitis ohne Polyposis nasi (CRSsNP) angegeben. Es wird jedoch darauf hingewiesen, dass eine mangelnde Besserung nicht zwingend zur Operation führen muss und dass andere Therapieformen nach entsprechender Absprache mit dem Patienten grundsätzlich möglich sind.

\subsubsection{Patientenselektion und patientenzentrierte Entschei- dungsfindung}

Eine chirurgische Intervention ist grundsätzlich dann eine geeignete oder angemessene Option, wenn bei der Abwägung von Risiken und Nutzen für die Gesundheit der Nutzen überwiegt. Die Nasennebenhöhlenchirurgie wird in der Regel als Therapieoption in Erwägung gezogen, wenn es nach erfolgter konservativer Therapie zu keiner Besserung der Beschwerden gekommen ist [11]. Auch wenn die medikamentöse Therapie die Grundlage für die langfristige Kontrolle der Erkrankung darstellt, konnten Studien zeigen, dass eine zeitgerechte chirurgische Intervention das klinische Ergebnis möglicherweise verbessern kann [34-36] (s. auch Abschnitt Zeitpunkt der Ope- ration). Problematisch ist, dass harte evidenzbasierte Kriterien zur Selektion von Patienten fehlen, die sicherstellen, dass Patienten von einer Operation profitieren werden. Des Weiteren hat sich gezeigt, dass radiologische Befunde der CRS nicht immer mit den Beschwerden der Patienten und der Lebensqualität korrelieren [37-44].

Der bereits im vorherigen Abschnitt genannte Artikel sieht nach internationalem Expertenkonsens die Nasennebenhöhlenchirurgie bei Patienten mit unkomplizierter CRS als Therapieoption, wenn die folgenden Kriterien erfüllt sind:

- Lund-Mackay-Score $\geq 1$

- Medikamentöser Therapieversuch mit topischem Steroid und systemischem Steroid bei CRSwNP und Breitspektrum/ antibiogrammgerechter antibiotischer Therapie (2-3 Wochen) oder niedrigdosierte Langzeittherapie mit Makroliden bei CRSsNP

- SNOT-22-Score (Sino-nasal Outcome Test) nach konservativer Therapie von $\geq 20$ [33] 
Eine Monotherapie mit topischen Steroiden oder systemischen Steroiden wurde als unsichere Indikation gewertet, d.h. hier konnte kein Konsens in der Bewertung zwischen den Experten erreicht werden. Die topische Therapie mit großvolumigen Nasenspülungen mit isotoner oder hypertoner Salzlösung wird als mögliche Ergänzung zur topischen Steroidtherapie gesehen. Es werden zudem Ausnahmesituationen diskutiert, in denen die Indikation zur Nasennebenhöhlenchirurgie gestellt werden kann, auch wenn die o.g. Kriterien nicht erfüllt werden und trotzdem ein Nutzen für den Patienten zu erwarten ist.

Es wurde versucht, diese Kriterien zu validieren, auch wenn dies methodisch nur eingeschränkt möglich ist, da die Kontrollgruppe in der durchgeführten Studie sehr klein war und sich eine echte Kontrollgruppe aus ethischen Gründen verbietet [45]. Es hat sich zudem gezeigt, dass in fast einem Drittel der Fälle aus unterschiedlichen Gründen von diesen Kriterien abgewichen wurde [46]. Es findet sich kein einheitlicher Grund für diese Abweichung, vielmehr spiegelt sich am ehesten die Komplexität der Entscheidungsfindung bei der Indikationsstellung in den Ergebnissen wider. Das Abweichen von den vorgegebenen Kriterien führt zu keinem Unterschied im subjektiven und objektiven Ergebnis (SNOT-22, Lund-Kennedy-Score, allgemeine Gesundheit). Es wird nötig sein, diese Kriterien in der Zukunft weiter zu modifizieren und zu präzisieren, insbesondere auch hinsichtlich der präoperativen medikamentösen Therapie.

Ein zentrales Element des Qualitätsmanagements im Gesundheitswesen ist die Patientenorientierung. Es ist wichtig, den Patienten in die Therapieentscheidung miteinzubeziehen. Mögliche Therapieoptionen mit den zu erwartenden Ergebnissen und Risiken werden mit dem Patienten erörtert, sodass dieser informiert eine Entscheidung treffen kann. Patientenpräferenzen und -erwartungen sollten in der Therapieentscheidung berücksichtigt werden. Die Entscheidung des Patienten für eine bestimmte Therapie (Fortführung der medikamentösen Therapie vs. Nasennebenhöhlenchirurgie) scheint v.a. durch die subjektive Beeinträchtigung der Patienten beeinflusst zu werden (Lebensqualität anhand SNOT-22, Rhinosinusitis Disability Index (RSDI)), wohingegen demografische und ökonomische Faktoren, Persönlichkeitsprofil, soziale Unterstützung, Vertrauen in den Operateur und objektive Kriterien, wie CT- und endoskopischer Befund und Riechvermögen, keine wesentliche Rolle zu spielen scheinen $[47,48]$. Eine stärkere Beeinträchtigung der Lebensqualität, insbesondere in den SNOT-Untergruppen psychologische Beeinträchtigung und Schlaf, führen häufiger zur Entscheidung für ein chirurgisches Vorgehen [48-51]. Inwieweit die Erwartungen der Patienten das Ergebnis nach Nasenebenhöhleneingriffen beeinflussen, ist aktuell unklar.

\subsubsection{Zeitpunkt der Operation}

Sowohl die zuvor beschriebenen Kriterien zur Selektion geeigneter Patienten für eine Operation der Nasennebenhöhlen, als auch das Europäische Positionspapier zur Rhinosinusitis und nasalen Polyposis (EPOS) sowie weitere nationale Leitlinien sehen eine nicht erfolgreiche medikamentöse Therapie als Voraussetzung für eine operative Intervention. Die Mindestdauer der medikamentösen Therapie wird im EPOS mit 12 Wochen, in den Konsensuskriterien mit 8 Wochen angegeben [11,33].

In mehreren Studien hat sich gezeigt, dass eine frühere chirurgische Intervention im Verlauf der CRS das postoperative Ergebnis verbessern kann. Patienten, die innerhalb von 12 Monaten nach Diagnose operiert werden, zeigen eine stärkere subjektive Verbesserung ihrer Symptome (SNOT-22). Andere Studien zeigen keinen Unterschied, bzw. ein besseres subjektives Ergebnis nach längerer Wartezeit [3436, 52-54]. Zusätzlich zeigt sich die Tendenz, dass die postoperative Verbesserung der Lebensqualität nach frühzeitiger Operation dauerhafter sein könnte. Patienten, die später im Verlauf der Erkrankung operiert werden, suchen postoperativ häufiger einen Arzt wegen typischen Beschwerden einer Sinusitis auf und erhalten häufiger Medikamente zur Behandlung der CRS [34, 36]. Es existieren verschiedene Hypothesen, weshalb eine frühere operative Intervention den Verlauf der chronischen Sinusitis beeinflussen könnte. Der bessere Zugang für und die bessere Wirksamkeit von Medikamenten, die Entfernung von den Krankheitsverlauf negativ beeinflussenden Faktoren (z. B. Biofilm, osteitischer Knochen) und Reduktion der Entzündungslast, könnten durch die Verhinderung tiefgreifender Gewebsschäden die Prognose verbessern [35]. Es zeigen sich zudem mehr Patienten mit Asthma in den Gruppen von Patienten, die später im Krankheitsverlauf operiert werden. Inwieweit die Reduktion der Entzündungslast der CRS durch die Nasennebenhöhlenoperation die Entwicklung eines klinisch manifesten Asthmas, analog zur Immuntherapie bei allergischer Rhinitis, beeinflussen könnte, ist noch nicht geklärt [34, 36, 55].

\subsubsection{Ambulant versus stationär}

Aktuell erfolgt die operative Versorgung von Patienten mit CRS vorwiegend stationär in Vollnarkose. Insbesondere in den USA und England zeigt sich in den letzten Jahren ein gegenläufiger Trend. Eingriffe an den Nasennebenhöhlen werden dort zunehmend ambulant und in Lokalanästhesie durchgeführt. Dies wird nicht zuletzt durch das Aufkommen minimalinvasiver Techniken, wie der Ballonsinuplastik und der Shaver-gestützten Polypektomie, ermöglicht $[56,57]$. Vorteile werden in Kostenersparnissen, einem geringeren Risiko nosokomialer Infektionen, geringeren Wartezeiten auf einen Operationstermin und einer erhöhten Patientenfreundlichkeit des ambulanten Vorgehens gesehen $[57,58]$.

Für ein ambulantes Vorgehen ist eine sorgfältige Selektion der Patienten notwendig. Patienten mit geringem Narkoserisiko, ohne relevante Risikofaktoren und Eingriffe mit umschriebenem Ausmaß und kurzer Dauer eignen sich prinzipiell am besten für ein ambulantes Vorgehen.

Eine Versorgung im Falle des Auftretens von Komplikationen muss gewährleistet sein, dies betrifft die postoperative Betreuung des Patienten und Erreichbarkeit eines Krankenhauses im Notfall. Die Häufigkeit einer ungeplanten stationären Aufnahme oder Wiedervorstellung nach ambulanter sinunasaler Chirurgie wird in der Literatur mit 0,8-8,8\% angegeben [58-62]. Die häufigsten Gründe hierfür sind Blutungen, Schmerzen, Übelkeit/Erbrechen/Dehydrierung und Fieber [59-63]. Mit einer vasovagalen Reaktion bei einem endoskopischen Eingriff in Lokalanästhesie muss in $0,16-0,6 \%$ der Fälle gerechnet werden $[64,65]$.

Eine stationäre Überwachung nach einer Operation der Nasennebenhöhlen, v.a. nach ausgedehnteren Eingriffen, ist sinnvoll, um mögliche zum Teil bedrohliche Komplikationen frühzeitig zu erkennen und behandeln zu können. Dies sind insbesondere Duraläsionen, eine Orbitaphlegmone oder ein Orbitahämatom und stärkere Nachblutungen aus dem Bereich der A. sphenopalatina und A. ethmoidalis anterior [12]. 


\subsubsection{Chirurgische und radiologische Checklisten}

Checklisten sind in chirurgischen Fächern weitverbreitet, werden von der WHO befürwortet und können durch Minimierung vermeidbarer Fehler, die Sicherheit der Operation und das Ergebnis verbessern [66-68].

In der Luftfahrtindustrie hat sich gezeigt, dass Checklisten möglichst kurz, einfach, klar und in der jeweiligen fachspezifischen Sprache formuliert sein sollten [69]. Es ist zudem wichtig, dass eine verbale Bestätigung möglich ist und ggf. entsprechende Korrekturmaßnahmen durchgeführt werden können.

Zusätzlich zur standardisierten WHO-Checkliste sollte das Vorhandensein der zum Patienten gehörigen CT-Bilder in der korrekten Seitenorientierung, bei geplanter Anwendung der Navigation die entsprechende Vorbereitung, die korrekte Beschriftung der Medikamente, die Funktionsfähigkeit der Absaugung und Elektrokoagulation und die Armierung von Tupfern überprüft werden [70].

Diverse Checklisten wurden für die präoperative Evaluation von CT-Bildern vor Nasennebenhöhleneingriffen entwickelt. Einige sind sehr detailliert, entsprechend aufwändig und schließen z.T. allgemeine Sicherheitsaspekte mit ein [70-76]. Die für die Nasennebenhöhlenchirurgie vorgeschlagenen Checklisten sind zum Teil evaluiert. Die Kenntnis der individuellen Anatomie und ggf. die Erkennung von individuellen anatomischen Besonderheiten im präoperativen CT, können durch diese CT-Checklisten verbessert werden $[71,73,75,76]$. Sie können zudem eine sinnvolle Ergänzung in der Lehre und Ausbildung von Assistenzärzten sein [75]. Allerdings steht der direkte Beweis aus, dass die Verbesserung der Kenntnis der individuellen Anatomie durch diese spezifischen Checklisten, auch tatsächlich die Komplikationsrate senkt. Zudem ist der Nutzen begrenzt, wenn die Checklisten unvollständig und in pflichtmäßiger Routine ausgefüllt werden [77].

\subsection{Operative Versorgung}

\subsubsection{Endoskopische, mikroskopische und konventionelle Nasennebenhöhlenchirurgie}

Die Technik der Endoskope und Videosysteme hat sich seit ihrer Einführung in die Nasennebenhöhlenchirurgie, bis hin zu den heutigen HD-Systemen und der 3D-Endoskopie, extrem verbessert. Das EPOS und die deutsche S2k-Leitlinie bezeichnen die endonasale endoskopische Chirurgie als den Standard der chirurgischen Therapie [6, 11]. Unter Berücksichtigung der geforderten Bildqualität mit entsprechend guter Detailsicht ist zudem die HD-Videoendoskopie als heutiger Standard anzusehen, wenn über den Monitor gearbeitet wird [12].

Die endoskopische Nasennebenhöhlenchirurgie ist seit Jahren Inhalt von Operationskursen, Operationslehren und der wissenschaftlichen Literatur. Alle relevanten Studien zu verschiedenen Aspekten der CRS behandeln die endonasale endoskopische Nasennebenhöhlenchirurgie.

Die endoskopische Chirurgie bietet im Vergleich mit dem Mikroskop einige wesentliche Vorteile. Die Weitwinkeltechnologie bewirkt eine gute Übersicht. Der Blick um die Ecke durch Winkeloptiken ermöglicht die direkte Sicht auf Pathologien, wie es mit dem Mikroskop nicht möglich ist. Das Arbeiten in 4-Hand-Technik ist nur mit dem Endoskop möglich und bringt große Vorteile bei der Chirurgie bei gutartigen und bösartigen Neubildungen der Nasennebenhöhlen. Die Arbeit über den Monitor ermöglicht eine präzise Erläuterung,
Anleitung und Kontrolle einzelner Operationsschritte in Lehre und Ausbildung.

Der Vergleich einer Operation der Kieferhöhle über den unteren Nasengang, der Kieferhöhlenoperation über den mittleren Nasengang mit Erweiterung des natürlichen Ostiums und einer CaldwellLuc-Operation zeigt bessere Ergebnisse beim Vorgehen über den mittleren Nasengang [78-80]. Ein Vergleich der konventionellen Chirurgie (Caldwell-Luc-Operation, Kieferhöhlenpunktion, intranasale Ethmoidektomie) mit der funktionellen endoskopischen Nasennebenhöhlenchirurgie (FESS) zeigt eine höhere Rate an vollständiger Symptomfreiheit bei einem endoskopischen Vorgehen [81].

Die chirurgische Genauigkeit bei der Durchführung verschiedener 2- und 3-dimensionaler motorischer Aufgaben ist in einer Untersuchung bei unerfahrenen Chirurgen mit dem Endoskop geringer als mit dem Mikroskop, bei erfahrenen Chirurgen zeigt sich kein Unterschied. Die Geschwindigkeit der Durchführung ist sowohl bei unerfahrenen als auch bei erfahrenen Chirurgen mit dem Endoskop geringer als mit dem Mikroskop [82]. In einer weiteren Studie zeigt sich bei der Durchführung motorischer Aufgaben im Modell, dass die Aufgaben mit der Kopflampe schneller als mit dem Endoskop oder Mikroskop durchgeführt werden können. Die Fehlerrate mit dem Kopflicht ist geringer als mit dem Endoskop und dem Mikroskop und mit dem Endoskop im Vergleich zum Mikroskop. Die Ergebnisse sind unabhängig von der Erfahrung mit dem jeweiligen optischen Medium [83, 84]. Die Visualisierung über die früheren Monitore ist im Vergleich zum direkten Blick durch die Optik schlechter [85, 86]. Bei der Bewertung dieser Studien ist zu berücksichtigen, dass sich die Visualisierung durch die HD-Technologie wesentlich verändert hat.

Der Vorteil des Mikroskops und der 3D-Endoskopie im Vergleich zur 2D-Endoskopie liegt in einer besseren Hand-Auge-Koordination, einer besseren Tiefenschärfe und Einschätzung von Größen und Entfernungen und soll so zu einer höheren Geschwindigkeit und Präzision und einer flacheren Lernkurve führen. Neue 3D-Systeme haben im Vergleich zu älteren Systemen eine bessere Ausleuchtung und höhere Auflösung [87]. In Studien zeigen sich unterschiedliche Ergebnisse mit z.T. besseren Ergebnissen unter Verwendung des 3DEndoskops, teilweise wiederum keine Unterschiede, v.a. bei erfahrenen Chirurgen $[88,89]$. Die höhere Genauigkeit in der Ausführung bestimmter Aufgaben unter Zuhilfenahme des 3D-Endoskopes durch Anfänger erklärt sich durch deren fehlende Erfahrung. Erfahrene Chirurgen wandeln durch die Bewegung des Endoskops, die Bewertung von relativen Größenverhältnissen, anatomische Kenntnisse und parallaktische Verschiebung, das 2-dimensionale Bild in eine mentale 3D-Information um. Die besonderen Vorteile der 3D-Systeme zeigen sich bisher v.a. im Labor $[90,91]$. Ein Nachteil ist die deutliche Anfälligkeit für Verschmutzungen der Linsen, v.a. in engen Kavitäten (z.B. vorderes Siebbein), was zur Verschlechterung bzw. Aufhebung des 3D-Eindrucks führt. Die 3D-Systeme sind noch recht neu und können deshalb bei weitem noch nicht als Standard angesehen werden.

Es existieren keine aktuelleren Studien, die die Komplikationsrate bei endoskopischer Chirurgie mit der Komplikationsrate bei mikroskopischer oder kombiniert mikroskopisch-endoskopischer Chirurgie vergleichen. Eine Metaanalyse zeigt keinen signifikanten Unterschied in der Häufigkeit leichter Komplikationen zwischen traditionellen endonasalen (mit Stirnlampe), mikroskopischen und endoskopischen Eingriffen. Bei den schwerwiegenden Komplikatio- 
- Tab. 2 Empfehlungen zum Einsatz der Navigation in der endoskopischen Nasennebenhöhlenchirurgie (nach [98]).

\title{
American Academy of Otolaryngology-Head and Neck Surgery
}

Revisionsoperationen

Veränderte Anatomie entwicklungsbedingt, postoperativ, posttraumatisch

Ausgeprägte Polyposis nasi

Pathologie der Stirnhöhle, des hinteren Siebbeins oder der Keilbeinhöhle

Erkrankungen mit Einbezug der Schädelbasis, Orbita, des N. opticus, der A. carotis interna

Schädelbasisdefekt und Rhinoliquorrhoe

Benigne und maligne sinunasale Neubildungen

\section{Empfehlungen australische Expertengruppe}

\author{
Empfohlen: \\ - Stereotaktische externe Lokalisation frontaler Pathologien \\ - Endoskopische Stirnhöhlenoperation bei vorheriger externer Stirnhöhlen- oder Siebbeinoperation \\ - Operation nach vorangegangener Rekonstruktion der Schädelbasis \\ - Pathologie mit Überschreiten der anatomischen Grenzen der Nasennebenhöhlen \\ - Benigne oder maligne Neubildungen mit Infiltration der Frontobasis \\ - Draf Typ III \\ Optional (wichtig): \\ - Ausgeprägte Polyposis nasi bei geplanter Pansinusoperation \\ - Stirnhöhlenrevisionsoperation \\ - Benigne und maligne Neoplasie der Nasennebenhöhlen ohne Infiltration der Schädelbasis \\ Optional (hilfreich): \\ - Revisionseingriffe \\ - Veränderte Anatomie entwicklungsbedingt, postoperativ, posttraumatisch \\ - Kongenitale Anomalien \\ - Chirurgische Ausbildung \\ - Spezifische Indikationen: Pädiatrische Fälle, zystische Fibrose, Keilbeinhöhleneingriff, Mukozele
}

nen zeigte sich jedoch ein signifikanter Unterschied zwischen mikroskopischen (2\%) und endoskopischen (1\%) Eingriffen [92].

\subsubsection{Navigation}

Der Einsatz eines Navigationssystems soll die intraoperative Orientierung des Operateurs, insbesondere in Fällen veränderter Anatomie, ausgeprägter Erkrankung und bei eingeschränkten Operationsbedingungen durch Blutungen, verbessern. Hierdurch sollen die Sicherheit und Vollständigkeit der Operation erhöht, das Ergebnis verbessert und die Revisionsrate gesenkt werden [93]. Es zeigen sich zudem Vorteile in der operativen Ausbildung [94, 95]. Die mentale Belastung bei Ärzten in der Ausbildung wird durch die Navigation nicht erhöht, sondern kann bei Ärzten mit etwas Erfahrung in der Nasennebenhöhlenchirurgie gesenkt werden [96, 97]. Ein Navigationssystem darf jedoch niemals die profunde Kenntnis der Anatomie ersetzen.

Empfehlungen zum möglichen Einsatz der Navigation im Rahmen von Nasennebenhöhleneingriffen wurden von der American Academy of Otolaryngology - Head and Neck Surgery und von einer australischen Expertengruppe veröffentlicht ( $\triangleright$ Tab. 2) [98]. Es wird in diesen Empfehlungen jedoch betont, dass letztendlich die Entscheidung zum Einsatz der Navigation im Ermessen des Operateurs liegt.

Die meisten publizierten Studien zur Komplikationshäufigkeit und zu Ergebnissen nach computergestützter endoskopischer Nasennebenhöhlenchirurgie sind retrospektive Studien, die Komplikationen und Ergebnisse vor und nach Einführung eines Navigationssystems vergleichen. Zudem wurden die Operationen z. T. durch Ärzte in der Ausbildung durchgeführt. Dies stellt eine methodische Einschränkung dar, da auch die wachsende Erfahrung des Operateurs eine Rolle spielt. In einigen Studien sind die Studiengruppen mitunter sehr inhomogen, was das Ausmaß der Operation und des Eingriffs angeht. Die meisten Studien zeigen keinen Unterschied hinsichtlich der Komplikationen mit und ohne Navigationssystem [93, 98-102]. In wenigen Studien zeigen sich eine Reduktion der Gesamtrate von Komplikationen und von schweren Komplikationen $[98,103]$. Zwei Studien zeigen eine Zunahme von schweren Komplikationen bzw. von Orbitaverletzungen bei Verwendung der Navigation, was auf ein aggressiveres Vorgehen der Operateure oder komplexere Eingriffe zurückgeführt wird [104, 105].

Ähnliches findet sich in Studien, die das Ergebnis von Nasennebenhöhleneingriffen mit und ohne Navigation vergleichen. Die meisten Studien zeigen keine signifikanten Unterschiede hinsichtlich der vollständigen Durchführung der geplanten Operation, der Revisionsrate, des postoperativen Lund-Mackay-CT-Scores und der Lebensqualität, gemessen anhand einer VAS, des Rhinosinusitis Outcome Measure (RSOM-31) oder des SNOT-20 [93, 98-101, 103]. Wenige Studien zeigen eine geringere Rate an Revisionen oder eine Verbesserung in der Sinusitis-spezifischen Lebensqualität in der Gruppe der navigationsgestützten Eingriffe [106, 107].

Eine Auswirkung des Einsatzes der Navigation auf Haftungsfragen 
in Gerichtsverhandlungen hat sich bisher nicht gezeigt [108, 109].

Gut designte Studien zur definitiven Klärung der Auswirkungen eines Navigationssystems auf die Komplikationsrate und das Ergebnis fehlen und sind aufgrund verschiedener Einschränkungen auch kaum möglich. Aufgrund der geringen Komplikationsraten bei der endoskopischen Nasennebenhöhlenchirurgie wäre die Fallzahl für eine statistisch aussagekräftige prospektive Studie extrem hoch. Außerdem ist eine Randomisierung von Patienten in eine Gruppe mit und ohne Navigation, abhängig von der Indikation, ethisch schwierig.

\subsubsection{Ausmaß der Operation/Radikalität}

Das Ausmaß der Nasennebenhöhlenchirurgie wird in der Regel an Art und Ausmaß der Erkrankung sowie die individuelle Anatomie angepasst. Chirurgische Techniken reichen von der reinen Polypektomie, der partiellen Uncinektomie über die FESS zur erweiterten radikaleren Chirurgie, die unter den Stichworten Nasalisation, RebootChirurgie oder Fullhouse FESS mit Draf III zu finden sind [110-113]. Individuelle Nomenklaturen wie MIST (Minimally Invasive Sinus Technique) sind Teil dieser Bandbreite möglicher Ausdehnungen des Eingriffes.

Die klassische FESS geht auf die Arbeiten von Messerklinger und die Annahme zurück, dass Entzündungsvorgänge zu einer Obstruktion der Ostien führen und es so zu einem Sekretstau in den Nasennebenhöhlen kommt, wodurch sich die Entzündung ausbreitet $[114,115]$. Seit dieser Zeit hat sich das Wissen über die Pathogenese und das therapeutische Vorgehen verändert. Gerade bei der CRSwNP ist klar, dass die Obstruktion der ostiomeatalen Einheit keine Rolle in der Entstehung und Aufrechterhaltung der Erkrankung spielt $[116,117]$. Ansätze zur endonasalen Chirurgie mit Erhalt der randständigen Mukosa zur Verbesserung der Schleimhautrehabilitation wurden bereits 1978 von Wigand und Steiner beschrieben [118]. Die FESS zielt auf den Erhalt gesunder Schleimhaut und von natürlichen anatomischen Drainagewegen, sowie die Verbesserung des mukoziliären Transports. Anatomische und/oder entzündliche Störfaktoren sollen beseitigt werden. Zusätzlich soll der Zugang für topische Medikamente verbessert werden [119].

Die ausschließliche Polypektomie kann zwar zeitweise eine Erleichterung der subjektiven Nasenatmungsbehinderung verschaffen, zeigt aber eine hohe Rezidivrate von 35\% in 6 Monaten und insgesamt 75\% [120, 121]. In einem großen englischen Kollektiv zeigt sich kein signifikanter Vorteil hinsichtlich der Verbesserung der Lebensqualität im SNOT-22 bei zusätzlicher Nasennebenhöhlenoperation im Vergleich zu einer reinen Polypektomie, jedoch ein leicht geringeres Risiko einer Revisionsoperation nach 36 Monaten und eine signifikante Reduktion dieses Risikos nach 5 Jahren [122, 123]. In einer Pilotstudie zeigt sich die ambulante endoskopische Polypektomie (EPIC) als eine mögliche kosteneffektive Behandlungsoption bei ausgewählten Fällen einer CRSwNP mit ausschließlich Nasenatmungsbehinderung [124]. Im kurz- und mittelfristigen Verlauf zeigt sich kein Unterschied zur konventionellen endoskopischen $\mathrm{Na}$ sennebenhöhlenchirurgie in der postoperativen krankheitsspezifischen Lebensqualität im SNOT-22 (Gesamtpunktzahl und Erreichen eines minimalen klinisch relevanten Unterschieds) in einem selektierten Patientengut $[122,125]$.

Bei der CRSsNP führt die Erweiterung des natürlichen Kieferhöhlenostiums zu einer größeren Offenheitsrate und einem besseren Lund-Mackay-Score als die reine Uncinektomie mit Belassen des na- türlichen Ostiums ohne Einfluss auf die Symptomausprägung $[126,127]$.

Die Stirnhöhlenchirurgie zählt zu den anspruchsvollsten Bereichen der Nebenhöhlenchirurgie. Das sinnvolle Ausmaß eines HNO-chirurgisch hergestellten Zuganges wird besonders in diesem Bereich immer wieder kontrovers diskutiert. Faktoren, die mit einer höheren Rezidivrate in Verbindung gebracht werden, sind CRSwNP, AERD, Asthma, hoher Lund-Mackay-Score, geringer a.p.-Durchmesser, Osteoneogenese, Ostitis, die Anzahl vorangegangener Operationen und eine inkomplette Entfernung von anterioren Siebbeinzellen, insbesondere Zellen, die in den Recessus frontalis ragen [112, 128-133]. Entsprechend wird die vollständige Entfernung aller Zellen im Recessus frontalis unter größtmöglicher Schonung der Schleimhaut empfohlen und ggf. bei Risikopatienten sogar die primäre Typ III-Drainage. Bei Patienten ohne Risikofaktoren, wie Asthma, AERD, CRSwNP und Ersteingriff wird ein konservativeres Vorgehen bevorzugt. Bei Vergleich einer Typ I- (reine anteriore Ethmoidektomie) mit einer Typ Ila-Drainage (Entfernung aller Zellen im Recessus frontalis) zeigen sich vergleichbare Ergebnisse hinsichtlich der Verbesserung der Lebensqualität und der Reduktion des Medikamentenverbrauchs [134]. In einem Vergleich einer Typ IIb- (unilaterale Entfernung des Stirnhöhlenbodens und des anterioren Anteils der mittleren Nasenmuschel) mit einer Typ III-Drainage (Entfernung des Stirnhöhlenbodens bds., des Stirnhöhlenseptums und eines superioren Nasenseptumanteils) zeigen sich vergleichbare Ergebnisse hinsichtlich der Verbesserung der Lebensqualität, des Erreichens einer minimalen klinisch relevanten Differenz im SNOT-22, der endoskopischen Offenheit der Stirnhöhle, der Komplikations- und Revisionsrate [135].

Ein kontrovers diskutiertes Thema ist weiterhin die partielle Resektion der mittleren Nasenmuschel. Die partielle Resektion ist u.a. bei diffusen polypösen Veränderungen indiziert oder wenn es aufgrund einer ausgeprägten Polyposis zu einer Atrophie der mittleren Nasenmuschel mit daraus resultierender Instabilität und Gefahr der postoperativen Lateralisation mit Vernarbung gekommen ist. Insbesondere bei Patienten mit einer subjektiv und objektiv ausgeprägten Erkrankung zeigt sich in den meisten Studien ein besseres Ergebnis nach partieller Resektion der mittleren Nasenmuschel mit weniger Adhäsionen und einer geringeren Rezidivrate, besseren Endoskopiebefunden und einer Verbesserung des Riechvermögens, was sich jedoch nicht in einer Verbesserung der Symptome und der Lebensqualität widerspiegelt [136-141]. Ein Vorteil hinsichtlich der krankheitsspezifischen Lebensqualität durch die partielle Resektion der mittleren Nasenmuschel ergibt sich lediglich bei Patienten nach Revisionschirurgie [142]. Es zeigen sich keine Symptome eines Empty Nose Syndromes und keine Einschränkungen des Riechvermögens nach partieller Resektion der mittleren Nasenmuschel [143].

Patienten mit CRSwNP, Asthma, Atopie und AERD (aspirin-exacerbated respiratory disease), ausgeprägter Erkrankung im CT und Ostitis zeigen in vielen Studien ein höheres Rezidivrisiko nach Nasennebenhöhlenoperationen. Die Erfolgsrate der Revisionschirurgie liegt bei 50-70\% [144, 145]. Ein kleiner Teil von Patienten bleibt jedoch trotz wiederholten Revisionseingriffen und optimaler medikamentöser Therapie symptomatisch. Dies führt zu dem Ansatz, dass bei diesen Patienten aufgrund der zugrundeliegenden ausgeprägten Entzündung, zusätzlich zur intensiven langfristigen medikamentösen Therapie, ein aggressiveres operatives Vorgehen indiziert ist. Ziel ist die maximale Reduktion der Entzündungslast mit Entfernung 
von Entzündungsmediatoren durch Entfernung von Polypen, ostitischem Knochen und Biofilmen und die Schaffung eines größtmöglichen Zugangs für die topische Therapie. In der Literatur werden diverse Zugänge zur weiten Eröffnung aller Nasennebenhöhlen beschrieben, mit ggf. medialer Maxillektomie (klassisch oder über prälacrimalen Zugang), Typ III-Drainage der Stirnhöhle und deren Modifikationen und radikaler Ethmoidektomie [110, 113, 145-148]. In bestimmten Fällen wird außerdem die primäre Anlage einer Draf Typ III-Drainage diskutiert [112]. Jedoch zeigt sich nicht in allen subjektiven und objektiven Messwerten ein besseres Ergebnis bei einem aggressiveren Vorgehen [112, 145, 149-152].

Neuere Konzepte beziehen aktuelle Forschungsergebnisse zur Pathogenese der CRS mit ein. Das Konzept der Reboot Surgery bei CRSwNP mit TH2-Entzündung besteht in der Entfernung der gesamten entzündeten Schleimhaut, um eine Re-Epithelialisierung mit funktionsfähiger Nasenschleimhaut zu ermöglichen. Alle Polypen und die gesamte Mukosa werden unter Erhalt des Periosts entfernt. Dies wird ggf. mit einer Draf Typ III- Drainage kombiniert. Es zeigen sich signifikant geringere Raten einer Rezidivpolyposis, eine längere rezidivfreie Zeit und geringere Werte im SNOT-22 bei der RebootChirurgie im Vergleich zur konventionellen FESS [111].

Es existiert kein allgemein akzeptierter Standard der Nasennebenhöhlenchirurgie. Die funktionelle Nasennebenhöhlenchirurgie wird in vielen Leitlinien, Operationslehren und aktuellen Publikationen als Goldstandard angesehen $[6,11]$. Studien, die nicht zwischen CRSsNP und CRSwNP unterscheiden, zeigen häufig keinen Unterschied zwischen zurückhaltender und aggressiverer chirurgischer Therapie. Bei der CRSwNP, mit ggf. weiteren assoziierten Erkrankungen und insbesondere in der Revisionschirurgie, kann ein aggressiveres Vorgehen in Kombination mit einer fortgeführten medikamentösen Therapie zu besseren subjektiven und objektiven Ergebnissen führen. Neue Erkenntnisse zur Pathogenese der CRS werden aller Voraussicht nach nicht nur die medikamentöse Therapie, sondern auch die chirurgische Therapie beeinflussen.

\subsubsection{Ausbildung des Operateurs, Chirurgen-spezifische Faktoren}

Die Qualität in der ärztlichen Patientenversorgung beginnt mit der Ausbildung der Operateure. Das Erlernen der endonasalen endoskopischen Nasennebenhöhlenchirurgie zeigt wie bei den meisten Operationstechniken eine Lernkurve. In einer Studie zur mikro-endoskopischen Nasennebenhöhlenchirurgie reduziert sich die Komplikationsrate deutlich nach 100 Eingriffen [153]. In einer neueren Studie wird für die kompetente Durchführung einer Uncinektomie, Kieferhöhlenoperation und vorderen Ethmoidektomie eine Fallzahl von durchschnittlich 23,1, für die posteriore Ethmoidektomie und Sphenoidotomie 22,5 und die Stirnhöhlenoperation 33 bestimmt [154]. Im Vergleich dazu werden chirurgische Lernkurven in der Literatur für otolaryngologische Eingriffe wie folgt angegeben: Cochlear Implant 30 Eingriffe, Septumplastik 12-23 und endoskopische Tympanoplastik 50-60 Eingriffe [155-158].

Es leuchtet ein, dass die selbstständige Durchführung der Operation unter Aufsicht eine größere Auswirkung auf die Lernkurve hat, als die alleinige Beobachtung und Assistenz [159]. Die Operation durch Ärzte in der Ausbildung unter Aufsicht eines erfahrenen Chirurgen scheint im Verlauf des „Lernprozesses“ keine signifikante
Erhöhung der Komplikationsrate und Verschlechterung des Ergebnisses nach sich zu ziehen [160-162].

Es existieren nur wenige Studien, die sich mit den Erfordernissen der chirurgischen Ausbildung in der endoskopischen Nasennebenhöhlenchirurgie befassen. Eine Befragung unter Teilnehmern verschiedener Dissektionskurse mit unterschiedlicher chirurgischer Erfahrung zeigt, dass die posteriore Ethmoidektomie und die Stirnhöhlenchirurgie die größten Schwierigkeiten bereiten [163]. Während des Erlernens der Technik werden nicht nur das Handling des Endoskops und der Instrumente als problematisch eingestuft (v.a. bei Winkeloptiken), sondern v.a. die räumliche Orientierung mit Übertragung der erlernten 2-dimensionalen Anatomie aus Lehrbüchern und des Computertomogramms (CTs) auf den dreidimensionalen Patienten, die Erkennung der bekannten Anatomie im endoskopischen Bild und die Beurteilung der Position des Endoskops und der Instrumente [163, 164].

Die Präparation an humanen Präparaten wird von Teilnehmern von Dissektionskursen als sehr hilfreich eingestuft. Die wiederholte Präparation im Rahmen eines Dissektionskurses führt zu einer vollständigeren Durchführung der Präparationsschritte [165]. Es scheint wichtig, sowohl die kognitiven (z.B. Abfolge einzelner OP-Schritte, durch Fachliteratur und Videos), als auch die manuellen Fähigkeiten (durch Übung am Simulator, Modell oder Körperspender) zu trainieren [166]. Aufgrund der eingeschränkten Verfügbarkeit von Körperspendern wurden verschiedene tierische, künstliche sowie Virtual Reality-Modelle und -Simulatoren entwickelt [159, 167-173]. Ziel ist es, die Lernkurve aus dem Operationssaal herauszunehmen und so potentiell die Komplikationsrate bei Patienten zu verringern. Modelle sind geeignet, grundsätzliche Fähigkeiten der Handhabung von Endoskopen und Instrumenten zu vermitteln [168, 170, 174]. Das Training an Simulatoren ist insbesondere für Assistenzärzte zu Beginn der Ausbildung sinnvoll. Es zeigt sich eine Lernkurve mit einem Plateau, das zu 80-90\% dem erfahrener Nasennebenhöhlenchirurgen entspricht und auch über längere Zeit ohne Übung erhalten bleibt [175]. Assistenzärzte, die an Modellen trainieren, zeigen eine schnellere Ausführung einzelner Operationsschritte, eine größere Geschicklichkeit und eine größere Sicherheit beim Umgang mit dem Endoskop und dem Instrumentarium im Operationssaal $[166,168,176]$. Es ist allerdings unklar, ob durch das Training am Simulator die Komplikationsrate gesenkt und somit die Qualität der Patientenversorgung verbessert wird $[177,178]$.

In einigen akkreditierten Ausbildungsprogrammen, z.B. in den USA, wird gefordert, die chirurgische Leistung der Assistenzärzte zu bewerten. Die subjektive Evaluation am Ende einer Rotation durch die ausbildenden Ärzte zeigt eine schlechte Reliabilität und Validität, wie dies bei einer retrospektiven Bewertung zu erwarten ist [179]. So wurden verschiedene Instrumente zur objektiven und strukturierten Bewertung der chirurgischen Leistung entwickelt (Objective Structured Assessment of Technical Skills = OSATS) $[180,181]$. Sie sind eine Form des in Deutschland in der Prüfung von Medizinstudenten verbreiteten OSCE (Objective Structured Clinical Examination). Die Kritik an diesen Instrumenten besteht darin, dass die Qualität des Ergebnisses nicht ausreichend beurteilt wird [182]. Es existieren wenige spezifisch für die endoskopische Nasennebenhöhlenchirurgie entwickelte Bewertungsinstrumente. Ein in England entwickeltes Verfahren beurteilt den Umgang mit Instrumenten und 
dem Endoskop, die anatomische Orientierung, Teamwork, das operative Vorgehen und das Management von Komplikationen [183]. Eine Version aus den USA beinhaltet einen ersten Teil zur Bewertung der Durchführung der einzelnen Operationsschritte und einen weiteren zur Beurteilung des Gesamtverständnisses, wie z.B. der Indikation, der Auswertung des CTs und des Umgangs mit dem Endoskop und den Instrumenten [184, 185]. Das GRESS (Global Rating of Endoscopic Surgical Skills) berücksichtigt zusätzlich zu den endoskopischen Fähigkeiten auch die Vorbereitung des Patienten und der Ausrüstung [186]. Sowohl Ausbilder als auch Assistenzärzte bewerten den Einsatz positiv. Es zeigt sich eine gute Validität und Reliabilität.

Bei Operationen anderer chirurgischer Fachgebiete, z. B. Pankreasresektion, Karotisendarteriektomie und gastrointestinale Tumorchirurgie, hat sich gezeigt, dass die Ergebnisqualität, gemessen durch Patient Reported Outcome Measures (PROMs) sowie Morbiditätsund Mortalitätsraten zwischen Chirurgen und Institutionen variieren können [187-189]. In diesem Zusammenhang wird auch die sog. Volume-Outcome-Hypothese auf der Ebene der Kliniken und des einzelnen Chirurgen immer wieder diskutiert. Für einige komplexe chirurgische Eingriffe, insbesondere in der Abdominal, Herz- und Gefäßchirurgie, zeigen sich geringere Morbidität- und Mortalitätsraten in Krankenhäusern mit einer höheren Fallzahl. Dies kann z.T. bei Unterscheidung zwischen retrospektiven und prospektiven Studien, nach Risikoadjustierung und abhängig von der Art des Eingriffs nicht mehr bestätigt werden $[187,188]$. Auch die Auswirkung der Fallzahl des einzelnen Chirurgen und eine Spezialisierung auf die Ergebnisqualität werden diskutiert. Nicht nur die Anzahl der Operationen, sondern auch Erfahrung und Alter der Chirurgen können das Ergebnis beeinflussen, mit einem z. T. erhöhten Mortalitäts- und Morbiditätsrisiko bei sehr erfahrenen Chirurgen, die mehr als 20 Jahre in ihrem Fachbereich tätig sind [190-192]. Eine Spezialisierung zeigt in einigen Studien einen Vorteil hinsichtlich des Ergebnisses [187, 193-195]. Es erscheint sinnvoll, dass weitere Faktoren, wie die Qualität der chirurgischen Leistung und ggf. adjuvante Therapien, die für das Ergebnis eine Rolle spielen können, in entsprechenden Betrachtungen berücksichtigt werden sollten [196, 197].

Es existieren nur wenige Studien, die die Ergebnisse der Nasennebenhöhlenchirurgie verschiedener Institutionen und Chirurgen vergleichen. Entsprechend bleibt unklar, welche chirurgenspezifischen Faktoren einen Einfluss auf das postoperative Ergebnis haben können. Zwei US-amerikanische Studien zeigen, vor Kontrolle patientenspezifischer Faktoren, einen Unterschied des postoperativen SNOT-22 und RSDI (Rhino-Sinusitis Disability Index) bzw. der Revisionshäufigkeit zwischen verschiedenen Institutionen bzw. Chirurgen $[198,199]$. Nach Kontrolle patientenspezifischer Faktoren sind diese Unterschiede z.T. nicht mehr signifikant. Um ein Benchmarking von Chirurgen bzw. Institutionen zu ermöglichen, ist eine Risikoadjustierung notwendig, um Unterschieden in den Patientenkollektiven einzelner Gebiete, Chirurgen und Kliniken Rechnung zu tragen. Die Voraussetzung hierzu ist die Definition patientenspezifischer Einflussfaktoren auf das postoperative Ergebnis. Dies sollte am besten spezifisch für die gewünschte Kennzahl der Ergebnisqualität, z.B. PROMs, Revisionsrate oder Produktivität, erfolgen. Die aktuell verfügbaren Daten sind noch unzureichend.

\subsection{Postoperatives Ergebnis}

Das Ziel einer Therapie ist die Wiederherstellung, die Verbesserung oder die Erhaltung der Gesundheit eines Patienten. Zur Messung des Ergebnisses nach Nasennebenhöhlenchirurgie konnte bisher kein Goldstandard etabliert werden. Traditionell wurde das Ergebnis in Studien durch objektive Befunde, wie Endoskopie- und CT-Scores sowie Komplikations- und Revisionsraten, bewertet. Es zeigen sich jedoch sehr unterschiedliche Ergebnisse bezüglich der Korrelation zwischen objektiven (endoskopisches Ergebnis und CT-Befund) und subjektiven Befunden prä- und postoperativ, wobei die meisten Studien keine Korrelation nachweisen konnten [17, 18, 38-44, 200202].

Jede Erkrankung, aber auch jede Therapie, beeinflusst das Wohlbefinden des Patienten. Diese Beeinflussung kann durch die gesundheitsbezogene Lebensqualität gemessen werden [203, 204]. Sie wird in der Regel in den 3 Bereichen physische, psychische und soziale Funktionsfähigkeit bestimmt. Nicht alle vorhandenen Messinstrumente berücksichtigen alle 3 Bereiche. In den letzten Jahren nimmt die Messung der gesundheitsbezogenen Lebensqualität in der $\mathrm{Na}$ sennebenhöhlenchirurgie einen zunehmenden Stellenwert ein. Dies deckt sich mit der Patientenzentrierung des Qualitätsmanagements und dem Ziel, die Ergebnisqualität aus Sicht des Patienten zu messen. Die richtige Therapie soll dem richtigen Patienten zukommen. So kommt bei der Messung der Ergebnisqualität den „patient reported outcome meassures “ (PROM) eine wesentliche Bedeutung zu. In den USA wurde 2010 eigens das Patient-Centered Outcome Research Institute gegründet, zur Förderung der Forschung, die sich auf Ergebnisse konzentriert, die wichtig und bedeutend für den Patienten sind. Die englische Regierung hat 2009 die routinemäßige Sammlung von PROMs für 4 elektive Eingriffe - Hüft- und Knieendoprothesen, Hernioplastik bei Leistenhernien und Varizen-OP - implementiert. Im Rahmen des Medicare Health Outcome Survey (HOS) in den USA werden PROMs gesammelt, um sie in Qualitätsverbesserungsmaßnahmen, Pay for Performance-Programmen, öffentlicher Berichterstattung und zur Gesundheitsverbesserung einzusetzen. Alle Institutionen mit Medicare-Verträgen sind zur Teilnahme verpflichtet.

Im Gegensatz zu objektiven und leistungsbasierten Ergebnissen messen PROMs den Teil der Versorgung, der zu konkreten Verbesserungen des Gesundheitszustandes, der Produktivität und des generellen Wohlbefindens führt [205].

\subsubsection{Subjektive Beurteilung}

\subsubsection{Allgemeine Messinstrumente der gesundheitsbezoge- nen Lebensqualität}

Allgemeine Messinstrumente der gesundheitsbezogenen Lebensqualität bewerten eine Reihe von allgemeinen physischen und psychischen Symptomen und beschränken sich nicht auf eine spezifische Erkrankung. Verschiedene allgemeine Messinstrumente werden bei Patienten mit Rhinosinusitiden angewendet [205, 206]. Sie werden u.a. zur Kosten-Nutzwert-Analyse herangezogen. Der Vorteil dieser allgemeinen Messinstrumente ist, dass verschiedene Erkrankungen hinsichtlich ihrer Auswirkungen auf die Lebensqualität verglichen werden können. Zudem ist ein Vergleich mit der Normalbevölkerung möglich. Häufig werden in Studien allgemeine Messinstrumente zur Beurteilung des allgemeinen Gesundheitszustandes mit krankheitsspezifischen Fragebögen zur Rhinosinusitis kombi- 
niert. So können alle 3 Bereiche der gesundheitsspezifischen Lebensqualität (physisch, psychisch, sozial) berücksichtigt werden.

Der SF-36 (Short Form 36 Health Survey) ist das weltweit am häufigsten eingesetzte Instrument zur Messung der allgemeinen gesundheitsbezogenen Lebensqualität. Es existieren Referenzwerte für viele Erkrankungen. Er umfasst 8 Teilbereiche (körperliche Funktionsfähigkeit, physische Rollenfunktion, körperliche Schmerzen, allgemeine Gesundheitswahrnehmung, Vitalität, soziale Funktionsfähigkeit, emotionale Rollenfunktion, psychisches Wohlbefinden). Durch unterschiedliche Gewichtung der acht Teilbereiche können zwei Dimensionen (körperliche und seelische Gesundheit) berechnet werden. Der SF-6 D ist eine verkürzte Form, er umfasst 11 Items, die sich in 6 Dimensionen aufteilen. Die deutsche Version des SF-36 ist validiert. Er wird in Studien teilweise in Kombination mit dem SNOT-20 oder -22 eingesetzt. In Studien zeigt sich ein positiver Effekt der Nasennebenhöhlenchirurgie auf die Lebensqualität gemessen durch den SF-36 [53, 207-211].

Der European Quality of Life 5-dimensional questionnaire (EQ5D) wurde bei Patienten mit CRS validiert. Er kann in Kombination mit anderen krankheitsspezifischen oder allgemeinen Messinstrumenten eingesetzt werden. Er besteht aus einer Selbsteinschätzung der Mobilität, Selbstständigkeit, der Aktivitäten des täglichen Lebens, von Schmerz und Angst/Depression in 3 oder 5 Stufen (EQ-5D$3 \mathrm{~L}$ oder $-5 \mathrm{~L}$ ) sowie einer Selbsteinschätzung des Gesundheitszustandes auf einer visuellen Analogskala. Es zeigen sich Verbesserungen in der gesundheitsbezogenen Lebensqualität bei Patienten nach $\mathrm{Na}$ sennebenhöhlenchirurgie gemessen durch den EQ-5D, insbesondere in den Untereinheiten Schmerz, Angst/Depression und Aktivitäten des täglichen Lebens [212,213].

\subsubsection{Spezifische Messinstrumente der gesundheitsbezoge- nen Lebensqualität}

Die chronische Rhinosinusitis beeinflusst die gesundheitsbezogene Lebensqualität der betroffenen Patienten [214]. Wie im Abschnitt Patientenselektion bereits ausgeführt, ist die Einschränkung der Lebensqualität ein wesentlicher Faktor in der Therapieentscheidung der Patienten. Seit den 1990er Jahren wurden diverse spezifische Messinstrumente für die Messung der gesundheitsbezogenen Lebensqualität bei Patienten mit CRS entwickelt und validiert. Eine Übersicht der in Studien gebräuchlichen PROMs findet sich in \ Tab. 3. Sie sind nur vereinzelt in einer validierten deutschen Version verfügbar. In einer Übersichtsarbeit mit Beurteilung der Qualität der Entwicklung und der psychometrischen Eigenschaften verschiedener PROMs, werden der SNOT-22 (22-item Sinonasal Outcome Test), der QOD (Questionnaire of Olfactory Disorders) und der SCT (Sinusitis Control Test) am besten bewertet [205].

Trotz der Validierung und des Einsatzes der vorhandenen spezifischen PROMs in zahlreichen Studien, gibt es Einschränkungen in und Kritik an der Wertigkeit für den Einsatz im klinischen Alltag. Keiner der verfügbaren Fragebögen beinhaltet Bereiche, die die Werte und Präferenzen der Patienten hinsichtlich bestimmter Behandlungsoptionen und den Einfluss bestimmter Komorbiditäten, wie der allergischen Rhinitis und des Asthma bronchiale bewerten [205].

Der Questionnaire of Olfactory Disorders (QOD) besteht aus 25 Elementen, die mit 0-3 Punkten bewertet werden und die in drei Bereiche eingeteilt werden können (positive, negative und soziale Elemente). Er wurde 2012 validiert. Eine gekürzte modifizierte Varian- te mit 17 negativen Aussagen und möglichen 0-51 Punkten wurde ebenfalls validiert und in weiteren Studien eingesetzt. In Studien zeigen sich schlechtere präoperative Ergebnisse bei Patienten mit Allergien, Steroidabhängigkeit und CRSwNP und eine signifikante Verbesserung nach Nasennebenhöhlenchirurgie, v.a. bei Fragen, die mit der Nahrungsaufnahme assoziiert sind [209, 215-217]. Die postoperativen Veränderungen des QOD korrelieren mit dem präoperativen CT-Befund, dem Gesamtwert des Sniffin' Stick-Tests (Riechschwelle, Diskrimination und Identifikation) und Fragen zum Riechvermögen in krankheitsspezifischen Lebensqualitätsfragebögen, wie dem SNOT-22 (22-item Sinonasal Outcome Test) und RSDI (Rhino-Sinusitis Disability Index), jedoch nicht mit objektiven Befunden des SIT40 (40 item Smell Identification Test) [215, 218].

Der Sinusitis Control Test (SCT) untersucht die Kontrolle der CRS unter der aktuellen medikamentösen Therapie. Er beinhaltet vier Fragen zu Symptomen der CRS (Nasenatmungsbehinderung, Rhinorrhoe), der Alltagsbeeinträchtigung und dem Medikamentenverbrauch in den dem Test vorangegangenen 2 Wochen. Die maximale Punktzahl sind 16 Punkte und anhand des Ergebnisses wird die CRS in eine kontrollierte, teilweise kontrollierte und unkontrollierte Form eingeteilt [219].

Der Chronic Sinusitis Survey (CSS) und der Rhino-Sinusitis Disability Index (RSDI) sind häufig eingesetzte Instrumente bei englischsprachigen Patienten. In vielen Studien werden sie gemeinsam angewendet. Ihre Reliabilität, Validität und Veränderungssensitivität wurden nachgewiesen. Der RSDI vereint die Bewertung des allgemeinen Gesundheitszustandes mit krankheitsspezifischen Fragen. Der CSS misst Sinusitis-spezifische Symptome und den Medikamentengebrauch in den vorangegangenen 8 Wochen. Der Nachteil des CSS ist, dass das Riechvermögenen nicht bewertet wird. Es wird auBerdem nicht die Schwere der Beeinträchtigung, sondern die Dauer des Bestehens der Symptomatik erfragt. Sowohl anhand des CSS, als auch des RSDI konnte gezeigt werden, dass die Nasennebenhöhlenchirurgie die Lebensqualität der Patienten verbessert und im Vergleich zur medikamentösen Therapie eine größere Verbesserung erreicht werden kann [213, 220, 221]. Es zeigen sich stabile Ergebnisse 6, 12 und 20 Monate postoperativ [222]. Nach Revisionsoperationen zeigen sich geringere Verbesserungen im CSS und RSDI als nach Primäreingriffen [210,223].

Der 31-item Rhinosinusitis Outcome Measure (RSOM-31) vereint eine krankheitsspezifische und allgemeine Lebensqualitätsmessung. Der SNOT-20 ist eine gekürzte Modifikation des RSOM-31. Der SNOT22 wiederum ist eine Erweiterung des SNOT-20, der 2 weitere Kardinalsymptome der CRS, die Nasenatmungsbehinderung und Riechminderung, abbildet. Sowohl der SNOT-20 als auch der SNOT-22 existieren in einer validierten deutschen Version ( $\bullet$ Tab. 4) (zum Zeitpunkt der Verfassung des Referates befand sich der SNOT-22 in Validierung). Beide können in 4 bzw. 5 Teilbereiche unterteilt werden, die sowohl durch die unterschiedlichen Subtypen der CRS, als auch durch die chirurgische bzw. medikamentöse Therapie unterschiedlich stark beeinflusst werden [224-226]. Der SNOT-22 kann in $3 \mathrm{Na}-$ sennebenhöhlen-spezifische Symptombereiche (rhinologische, extranasale rhinologische, Ohr/faziale Symptome) und 2 allgemeine gesundheitsbezogene Lebensqualitäts-Bereiche (Schlaf-, psychologische Beeinträchtigung) unterteilt werden [226]. Bei der Unterteilung in 4 Teilbereiche werden nasale, otologisch/faziale, emotionale Symptome und Beeinträchtigungen des Schlafs unterschieden [227]. 
- Tab. 3 Gebräuchliche Messinstrumente des patient reported outcome adaptiert nach [205, 206].

\begin{tabular}{|c|c|c|c|c|c|}
\hline Messinstrument & $\begin{array}{l}\text { Anzahl der } \\
\text { Fragen }\end{array}$ & Teilbereiche & $\begin{array}{l}\text { Punkte- } \\
\text { spanne }\end{array}$ & $\begin{array}{l}\text { Validierung (Anzahl Pat. } \\
\text { Val.studie) }\end{array}$ & $\begin{array}{l}\text { Deutsche } \\
\text { validierte Version }\end{array}$ \\
\hline $\begin{array}{l}\text { Chronic Sinusitis Survey } \\
\text { (CSS) } 1995\end{array}$ & 6 & $\begin{array}{l}\text { CRS Symptome } \\
\text { Medikamentengebrauch }\end{array}$ & $0-100$ & ja (104) & nein \\
\hline $\begin{array}{l}\text { Rhinosinusitis Disability } \\
\text { Index (RSDI) } 1997\end{array}$ & 30 & $\begin{array}{l}\text { Physisch } \\
\text { Funktionell } \\
\text { Emotional }\end{array}$ & $0-120$ & ja (87) & $\begin{array}{l}\text { Rhinosinusitis- } \\
\text { Behinderungs-Index }\end{array}$ \\
\hline $\begin{array}{l}\text { Rhinosinusitis Severity } \\
\text { Inventory (RSI) } 2003\end{array}$ & 20 & $\begin{array}{l}\text { CRS Symptome } \\
\text { Medikamentengebrauch } \\
\text { Arbeit und Soziales }\end{array}$ & $0-100$ & ja (322) & nein \\
\hline $\begin{array}{l}\text { Rhinosinusitis Quality of } \\
\text { Life Survey (RhinoQoL) } \\
2005\end{array}$ & 30 & $\begin{array}{l}\text { Schwere der Symptomatik } \\
\text { Beeinträchtigung } \\
\text { Auswirkungsskala }\end{array}$ & $0-100$ & ja (49) & nein \\
\hline $\begin{array}{l}\text { Sino-Nasal Outcome Test } \\
16 \text { (SNOT-16) } 1999\end{array}$ & 16 & na & $0-48$ & ja (47) & nein \\
\hline $\begin{array}{l}\text { 31-item Rhinosinusitis } \\
\text { Outcome Messure } \\
\text { (RSOM-31) } 1995\end{array}$ & 31 & $\begin{array}{l}\text { Nasal } \\
\text { Auge } \\
\text { Ohr } \\
\text { Schlaf } \\
\text { Allgemein } \\
\text { Emotional } \\
\text { Funktionell }\end{array}$ & $0-155$ & ja (142) & nein \\
\hline $\begin{array}{l}\text { Sino-Nasal Outcome Test } \\
20 \text { (SNOT-20) } 2002\end{array}$ & 20 & na & $0-100$ & ja (102) & ja \\
\hline $\begin{array}{l}\text { Sino-Nasal Outcome Test } \\
22 \text { (SNOT-22) } 2009\end{array}$ & 22 & $\begin{array}{l}\text { Rhinologisch } \\
\text { Extranasal rhinologisch } \\
\text { Ohr/fazial } \\
\text { Psychologisch } \\
\text { Schlaf }\end{array}$ & $0-110$ & ja (2803) & $\begin{array}{l}\text { ja (wird aktuell } \\
\text { validiert) }\end{array}$ \\
\hline $\begin{array}{l}\text { Questionnaire of Olfactory } \\
\text { Disorders (QOD) } 2012\end{array}$ & 25 & $\begin{array}{l}\text { Negative Elemente } \\
\text { Positive Elemente } \\
\text { Soziale Elemente }\end{array}$ & $0-57$ & ja (102) & nein \\
\hline $\begin{array}{l}\text { Sinusitis Control Test (SCT) } \\
2015\end{array}$ & 4 & $\begin{array}{l}\text { Symptome } \\
\text { Produktivität } \\
\text { Gebrauch von Rescue-Medikamenten }\end{array}$ & $0-16$ & ja (15) & nein \\
\hline
\end{tabular}

Da die englische Version des SNOT-22 im Gegensatz zum SNOT-20 zusätzlich die beiden Kardinalsymptome nasale Obstruktion und Geruch beurteilt, sollte er dem SNOT-20 vorgezogen werden. Beide Symptome finden sich häufig bei Patienten mit CRS und tragen wesentlich zu einem Therapiewunsch der Patienten bei. Die deutsche adaptierte Version des SNOT-20 enthält im Gegensatz zur englischen Version Fragen zur Riechminderung und Nasenatmungsbehinderung, nachdem 2 Fragen zum Schlaf gestrichen wurden.

In einem Vergleich einzelner Instrumente zeigt sich eine sehr gute Korrelation zwischen den Ergebnissen des RSDI und des SNOT-22, allerdings nur eine mäßige Korrelation zwischen dem CSS und RSDI [228]. Eine Kombination von RSDI und CSS kann sinnvoll sein, da auch der Medikamentengebrauch berücksichtigt wird. Eine Kombination des SNOT-22 mit dem RSDI ist aufgrund hoher Redundanz hingegen nicht sinnvoll.

Der SNOT-22 wurde hinsichtlich seiner psychometrischen Eigenschaften (Reliabilität, Validität und Veränderungssensitivität) in einer großen englischen Kohorte validiert [229].
Der SNOT-22 kann zur Beurteilung des Ergebnisses nach medikamentöser und chirurgischer Therapie herangezogen werden. Traditionell werden Unterschiede vor und nach einer Therapie als signifikant oder nicht signifikant angegeben. Dies berücksichtigt nicht, inwieweit diese Unterschiede auch tatsächlich zu klinisch relevanten bzw. wahrnehmbaren Unterschieden führen. Dies ist wichtig in der Interpretation der Veränderung der Ergebnisse über die Zeit bzw. nach einer bestimmten Therapie, inwieweit also eine Veränderung z.B. der Punktzahl eines Fragebogens auch tatsächlich eine Veränderung des Gesundheitszustandes anzeigt. Dies wird durch die minimale klinisch relevante Differenz (MCID = minimal clinically important difference) angegeben. Für den SNOT-22 wurde eine MCID von 8,9 für die Gesamtpunktzahl und 3,8 für die rhinologische, 2,4 für die extranasal rhinologische, 3,2 für die Ohr/faziale, 3,9 für die psychologische und 2,9 für die schlafbezogene Untereinheit ermittelt $[229,230]$. Dies bedeutet, dass eine Veränderung der Gesamtpunktzahl von weniger als 9 von Patienten nicht als tatsächliche Verbesserung oder Verschlechterung wahrgenommen wird. Studien zeigen, dass Patienten mit CRS durch die Nasennebenhöhlenchirurgie eine 
> Tab. 4 SNOT-22 (deutsche Version)

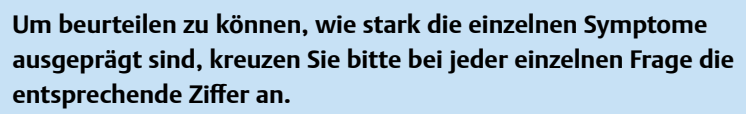

(n)

\begin{tabular}{|l|l|}
\hline 1. Schneuzen der Nase notwendig & 0 \\
\hline 2. verstopfte Nase / Behinderung der Nasenatmung & 0 \\
\hline 3. Niesreiz & 0 \\
\hline 4. Naselaufen & 0 \\
\hline 5. Husten & 0 \\
\hline 6. Sekretfluß in den Rachen & 0 \\
\hline 7. dickes schleimiges Nasensekret & 0 \\
\hline 8. Druckgefühl auf den Ohren & 0 \\
\hline 9. Schwindelgefühl & 0 \\
\hline 10. Ohrenschmerz & 0 \\
\hline 11. Gesichtsschmerz, Druckgefühl im Gesicht & 0 \\
\hline 12. Riechminderung / Geschmacksminderung & 0 \\
\hline 13. Probleme beim Einschlafen & 0 \\
\hline 14. Nächtliches Aufwachen & 0 \\
\hline 15. Mangel an gutem nächtlichem Schlaf & 0 \\
\hline 16. Müdigkeit beim Aufwachen & 0 \\
\hline 17. Erschöpfung & 0 \\
\hline 18. Verminderte Leistungsfähigkeit & 0 \\
\hline 19. Konzentrationsschwäche & 0 \\
\hline 20. Frustrationen/Rastlosigkeit/Reizbarkeit & 0 \\
\hline 21. Traurigkeit & 0 \\
\hline 22. Nebenhöhlenbeschwerden sind mir peinlich & \\
\hline
\end{tabular}
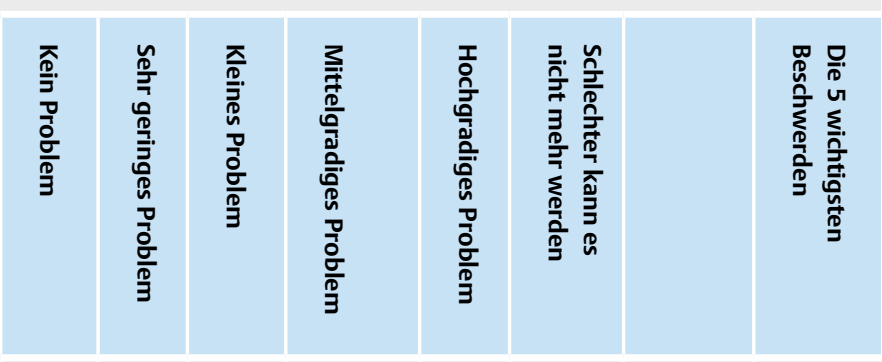

\begin{tabular}{|l|l|l|l|l|l|l|}
\hline 0 & 1 & 2 & 3 & 4 & 5 & 0 \\
\hline 0 & 1 & 2 & 3 & 4 & 5 & 0 \\
\hline
\end{tabular}

Bitte markieren Sie hier die 5 wichtigsten Beschwerden,

die Ihre Gesundheit beeinträchtigen (bitte maximal 5 Fragen ankreuzen)

klinisch relevante Verbesserung erreichen können. 64-80\% der Patienten erreichen eine MCID nach Nasennebenhöhlenchirurgie. Teilweise kann die zweifache minimale klinische Differenz erreicht werden. Patienten mit einer höheren Gesamtpunktzahl (> 30 bzw. 61-70 Punkte) präoperativ erreichen mit einer höheren Wahrscheinlichkeit eine minimale klinisch relevante Differenz des Gesamtpunktwertes postoperativ [35, 49, 53, 231-233]. Patienten mit höherem Haushaltseinkommen erreichen zudem mit höherer Wahrscheinlichkeit eine MCID [233] (Anm: US-amerikanische Studie mit entsprechenden Unterschieden im Gesundheitssystem).

In einer großen englischen Kohorte zeigt sich, dass Patienten mit CRSwNP im Vergleich zu Patienten mit CRSsNP präoperativ signifikant höhere Punktwerte im nasalen Testbereich angeben, wobei der Gesamtpunktwert bei beiden Gruppen keinen wesentlichen Unterschied zeigt. Frauen geben höhere Punktwerte an als Männer [35, 234]. Patienten mit CRSsNP zeigen vor einer Therapie höhere Punktwerte in den fazialen und emotionalen Untereinheiten. Auch eine schwedische Studie weist höhere Punktwerte in den Teilbereichen Riech- und Geschmacksminderung, Husten und Gesichtsschmerz/-druck bei Patienten mit CRSwNP im Vergleich zu Patienten mit CRSsNP nach [235]. Die geschlechtsspezifischen Unterschiede zeigen sich ebenfalls in weiteren Studien [233, 235-237]. Präoperativ geben Frauen höhere Werte in den rhinologischen und den extranasal rhinologischen Untereinheiten an. Nur in der frühen postoperativen Phase (3 Monate) zeigen Frauen weiterhin höhere Punktwerte als Männer. Wird der SNOT-22 bei gesunden Probanden angewendet, zeigen sich geringere Punktwerte als bei Patienten mit CRS und höhere Punktwerte bei Frauen als bei Männern [229, 238].

Nach Nasennebenhöhlenchirurgie zeigt sich eine signifikante Verbesserung des Gesamtpunktwertes bei Patienten mit CRSsNP und CRSwNP [49, 53, 230, 232, 233]. Trotz signifikanter Verbesserungen liegen die postoperativen Werte der Patienten mit CRS über den Werten gesunder Probanden [35]. Hinsichtlich der Untergruppen mit und ohne Polyposis nasi zeigen sich unterschiedliche Ergebnis- 
se. Einige Studien finden keinen signifikanten Unterschied zwischen der Verbesserung der Lebensqualität von Patienten mit CRSwNP versus CRSsNP [53, 239]. Andere zeigen eine größere Zunahme der Lebensqualität bei Patienten mit CRSwNP, auch wenn die absoluten Punktwerte i.d.R. immer noch unter denen von Patienten ohne Polyposis liegen [24, 35, 123, 232, 240, 241]. In einer neueren Metaanalyse zeigt sich kein signifikanter Einfluss von Alter, Geschlecht, Endoskopiebefund, CT-Befund, Polyposis, Rauchen, Depression und allergischer Rhinitis auf die postoperative Veränderung des SNOT22. Asthma, vorangegangene Operationen und ein höherer präoperativer Punktwert sind mit einer größeren postoperativen Veränderung, längeres Follow-up mit geringeren Veränderungen assoziiert, wobei nur Asthma, präoperativer SNOT-Wert und Länge des Followup nach weiterer Analyse signifikante Einflussfaktoren auf das postoperative Ergebnis darstellen [21]. In einer Studie zeigt sich ein negativer Einfluss von steroidabhängigen Erkrankungen (z.B. Autoimmunerkrankungen) [198].

Es zeigen sich stabile Ergebnisse der Gesamtpunktzahl sowie der Punktzahl der Teilbereiche zwischen 6 Monaten und bis zu 5 Jahren postoperativ [24, 53, 123, 242].

Es kommt v.a. zu einer Verbesserung der Nasennebenhöhlen-spezifischen Teilbereiche, während die Verbesserung in den psychologischen und schlafbezogenen Bereichen geringer ist bzw. Einschränkungen persistieren [51, 53, 232, 243-246]. Zusätzlich zu der in Lebensqualitätsfragebögen nachgewiesenen Beeinträchtigung des Schlafs, zeigt sich die Schlafqualität bei Patienten mit CRS, gemessen anhand des PSQI (Pittsburgh Sleep Quality Index), reduziert [244, 247-249]. Der PSQI verbessert sich zwar nach Nasennebenhöhlenchirurgie, bleibt jedoch z. T. deutlich über dem Wert gesunder Probanden [244, 248, 249]. Allerdings hat sich gezeigt, dass gerade höhere präoperative Punktwerte in den psychologischen und schlafbezogenen Bereichen die Therapieentscheidung hin zur Nasennebenhöhlenchirurgie beeinflussen [50,51]. Es scheint wichtig, Patienten hier entsprechend hinsichtlich der zu erwartenden Verbesserungen zu beraten.

Für die Beratung von Patienten hinsichtlich möglicher Therapieoptionen wäre es wünschenswert, die Wahrscheinlichkeit eines bestimmten postoperativen Ergebnisses einschätzen zu können. Die prädiktiven Eigenschaften des SNOT-22 wurden in mehreren Studien untersucht. Hier hat sich gezeigt, dass Patienten mit einem höheren präoperativen Gesamtpunktwert von > 30 eine größere postoperative Verbesserung und mit größerer Wahrscheinlichkeit eine MCID erreichen können [35, 49, 53, 232, 250]. Patienten mit Werten > 30 haben eine ca. 70-80\%ige Chance eine MCID zu erreichen. Außerdem ist eine Verbesserung des postoperativen SNOT-Wertes um ca. 40-50\% zu erwarten. Die größte Wahrscheinlichkeit, eine MCID zu erreichen, zeigt sich bei Patienten mit Werten zwischen 60 und 80. Hohe Punktwerte in den Fragen „Schneuzen der Nase notwendig“ und „Verstopfte Nase/Nasenatmungsbehinderung“ erwiesen sich als die prädiktivsten Elemente [53, 232, 250].

Der SNOT-22 wurde ebenfalls herangezogen, um die Wahrscheinlichkeit einer Revisionsoperation einzuschätzen. Hier zeigt sich bei Patienten, die präoperativ eine höhere Gesamtpunktzahl angeben, die nach 3 Monaten keine MCID erreichen und deren SNOT-22-Ergebnisse sich zwischen 3 und 12 Monaten postoperativ um mehr als eine MCID verschlechtern, ein höheres Risiko einer Revisionsoperation [35, 251]. Inwieweit eine intensivierte medikamentöse Thera- pie oder engmaschigere Kontrollen diese Entwicklung beeinflussen können, ist noch unklar.

Um ein Messinstrument der Lebensqualität als Kennzahl der Ergebnisqualität in ein Qualitätsverbesserungsvorhaben einzubringen, wäre es notwendig, Referenzwerte für die zu erwartende Verbesserung und individuelle Patientenfaktoren, die diesen Referenzwert verändern können, zu definieren. Ärzte könnten so z.B. ihre postoperativen SNOT-22-Ergebnisse, angepasst an ihr Patientengut, gegenüber einer Referenzpopulation vergleichen. Entsprechende Werte wurden bisher nur durch eine Metaanalyse definiert und nur die Einrichtung eines Patientenregisters würde zuverlässige Daten in entsprechendem Umfang liefern [21].

\subsubsection{Produktivität}

Die CRS führt nicht nur zu direkten Kosten für das Gesundheitssystem, sondern auch zu indirekten Kosten durch eine Reduktion der Produktivität der Arbeitnehmer, z.B. durch Krankheitstage. Produktivitätskosten werden errechnet durch Zusammenfassung der Kosten für Absentismus (Krankheitstage), Präsentismus (Reduktion der Arbeitsproduktivität durch Leistungseinschränkung von Beschäftigten, die auf gesundheitliche Einschränkungen wie z. B. chronische Erkrankungen zurückzuführen sind) und verlorener Zeit zu Hause. Sie scheinen mit der krankheitsspezifischen Lebensqualität und der subjektiven Symptomkontrolle assoziiert zu sein; je schlechter die Lebensqualität, insbesondere in den Teilbereichen Schlaf- und psychologische Beeinträchtigung, desto höher die Kosten bzw. die Anzahl an Krankheitstagen [1, 252, 253]. Patienten, die nach Nasennebenhöhlenchirurgie eine starke Verbesserung der krankheitsspezifischen Lebensqualität angeben, zeigen auch eine größere Steigerung der Produktivität [21]; bei den meisten Patienten zeigt sich jedoch eine postoperative Verbesserung der Produktivität unabhängig vom Grad der Verbesserung der Lebensqualität. Eine signifikante Korrelation zu einzelnen Teilbereichen des SNOT-22 besteht nicht.

In einer europäischen Studie geben Patienten mit CRS präoperativ durchschnittlich 8-14 Krankheitstage an. Nach Nasennebenhöhlenchirurgie zeigt sich eine z. T. signifikante Reduktion der Krankheitstage auf 1-7 Tage pro Jahr [53, 235]. Präoperativ geben $57 \%$ der Patienten Krankheitstage aufgrund von Beschwerden einer chronischen Sinusitis an, postoperativ sinkt diese Zahl auf $44 \%$.

In einer US-amerikanischen Studie zeigt sich eine Reduktion von Absentismus und Präsentismus durch die CRS von 63 auf 22 Tage. Die Produktivitätskosten bzw. der Produktivitätsverlust nehmen nach Nasennebenhöhlenchirurgie ebenfalls signifikant ab [21, 254]. Im Gegensatz dazu bleibt der Produktivitätsverlust nach fortgesetzter adäquater medikamentöser Therapie weitestgehend unverändert [21, 255]

\subsubsection{Objektive Beurteilung}

Auf die objektive Beurteilung des Ergebnisses der Nasennebenhöhlenchirurgie soll hier nur kurz ergänzend eingegangen werden, da im Rahmen der Beurteilung der Ergebnisqualität den subjektiven Beurteilungskriterien ein höherer Stellenwert zukommt.

\subsubsection{Endoskopische Bewertung}

Es existieren verschiedene endoskopische Bewertungssysteme zur Beurteilung der klinischen Befunde bei CRS. Das am häufigsten in 
rhinologischen Studien eingesetzte System ist das System nach Lund und Kennedy [256]. Beurteilt werden Polypen, Ödem, Krusten, Sekretion und Narbenbildung. Es werden jeweils Punkte von 0-2 vergeben. Es wurde entwickelt, um den endoskopischen Befund bei Patienten nach Nasennebenhöhlenchirurgie zu beschreiben. Da es nicht validiert wurde und eine geringe Korrelation mit PROMs zeigt, wurden modifizierte Versionen dieses Systems entwickelt, die eine bessere Interrater- und Retest-Reliabilität und Korrelation mit PROMs zeigen [201, 257]. Die Befunde Krusten und Narben wurden gestrichen und das modifizierte System ist auch zur Beurteilung von präoperativen Befunden anwendbar. Zahlreiche Studien zeigen eine signifikante Verbesserung des endoskopischen Befundes durch die primäre Nasennebenhöhlenchirurgie und Revisionschirurgie [121, 258-261]

Seltener genutzte Systeme sind das Perioperative Sinus Endoscopic scoring system (POSE) und das Discharge, Inflammation, Polyp scoring system (DIP) [262, 263]. Das DIP-System zeigt ebenfalls eine gute Reliabilität. Das POSE-System wurde zur Beurteilung von Patienten nach Nasennebenhöhleneingriffen entwickelt und zeigt eine schlechtere Reliabilität [257].

\subsubsection{Revisionsrate}

Die Angaben zu Revisionsraten nach Nasennebenhöhlenchirurgie variieren in der Literatur. Ursachen hierfür können in den z.T. geringen eingeschlossenen Patientenzahlen und dem kurzen Follow-up gesehen werden. In 2 groß angelegten englischen Studien wird eine Revisionsrate von $4 \%$ innerhalb eines Jahres und $11 \%$ nach 3 Jahren angegeben [24, 264]. 19,1\% der Patienten, die 5 Jahren nach der ursprünglichen Operation noch auf den Fragebogen antworteten $(52,2 \%)$, wurden in diesen Jahren erneut operiert [123]. Es zeigt sich eine höhere Revisionsrate bei Patienten mit CRSwNP. In einer großen populationsbasierten amerikanischen Studie zeigt sich eine Langzeit-Revisionsrate von 15,9\% [265]. Bei den meisten dieser Patienten erfolgt im Verlauf lediglich eine weitere Operation. Das Risiko einer Revisionsoperation ist bei männlichen und jüngeren Patienten geringer. In einer weiteren, ähnlich konzipierten amerikanischen Studie, zeigt sich eine Revisionsrate von 6,65\%, wobei das Revisionsrisiko bei Frauen auch in dieser Studie höher ist [266]. Ältere Langzeitstudien einzelner Operateure zeigen Revisionsraten von 18 und $21 \%[267,268]$.

Die Revisionsrate bei Patienten mit CRSwNP ist höher als bei Patienten mit CRSsNP [123, 199, 264-266, 269]. Weitere Risikofaktoren sind Asthma und eine Analgetikaintoleranz. In der vorhandenen Literatur variiert die Zeit bis zur Revisionsoperation von 1-10 Jahren $[265,269,270]$. In der zuvor erwähnten amerikanischen Studie lagen zwischen der primären Operation und der Revisionsoperation durchschnittlich 4,39 Jahre, wobei sich kein Unterschied zwischen Patienten mit CRSwNP und CRSsNP zeigte.

\subsubsection{Komplikationsrate}

Komplikationen nach Nasennebenhöhlenchirurgie werden in leichte und schwerwiegende Komplikationen eingeteilt [11]. Zu den schwerwiegenden Komplikationen zählen Schädelbasisverletzungen mit Liquorrhoe, intrakranielle Komplikationen, orbitale Komplikationen (z.B. Doppelbilder, Visusminderung) und ausgeprägte Blutungen. Adhäsionen, Hyposmie, Infektionen und leichtgradige Blutungen werden zu den leichten Komplikationen gezählt. Die endonasale endoskopische Nasennebenhöhlenchirurgie weist niedrige Komplikationsraten auf. Die Rate leichter Komplikationen wird mit 5-7\%, die der schwerwiegenden Komplikationen mit 0,4-1\% angegeben [12, 104, 105, 178, 271-274]. Als Risikofaktoren für eine höhere Komplikationsrate gelten:

Ausmaß der Erkrankung, Ausmaß der Polyposis, Ausmaß der Operation (nicht in [271]), anatomische Veränderungen, Voroperationen/fehlende Landmarken, rechtsseitige Operation bei rechtshändigen Operateuren, vermehrte Blutung und Komorbiditäten $[12,178,271,275]$.

Einige dieser Faktoren werden in der Literatur mitunter kontrovers diskutiert.

\subsubsection{Riechtestung}

Der gebräuchlichste Test des Riechvermögens ist der Test mittels Sniffin' Sticks. Es können die Riechschwelle, Diskrimination und Identifikation bestimmt und in einem Gesamtwert zusammengefasst werden. Es existieren altersabhängige Normwerte [276]. Weitere in Studien häufig verwendete Tests sind der SIT-40 (40 item Smell Identification Test, früher University of Pennsylvania Smell Identification Test (UPSIT)) mit 40 Riechstoffen und die verkürzte Version, der Brief Smell Identification Test (B-SIT) mit 12 Riechstoffen. Beides sind überschwellige Identifikationstests, für die alterabhängige Normwerte vorliegen [277, 278]. Die minimale klinisch relevante Differenz wurde für den SIT-40 mit $\geq 4$ und für den Gesamtwert des Sniffin' Sticks-Tests mit $\geq 5,5$ angegeben [279].

Die Angaben zur Verbesserung des Riechvermögens nach Nasennebenhöhlenchirurgie schwanken in der Literatur und sind nicht sicher vorhersehbar. Die Pathophysiologie der Riechminderung bei CRS ist nicht vollständig geklärt. Es werden neben mechanischen Ursachen auch Entzündungsvorgänge mit neuroepithelialer Schädigung vermutet, wodurch die schwankenden Verbesserungen nach Nasennebenhöhlenchirurgie erklärt werden. Patienten zeigen eine signifikante Verbesserung nach Nasennebenhöhlenoperationen in 23-68\% [261, 280-283]. Die Entfernung von Polypen aus der Riechspalte scheint nicht zu einer Riechminderung, sondern zu besseren postoperativen Ergebnissen zu führen [284, 285]. Patienten mit präoperativer Anosmie und mit CRSwNP erreichen mit größerer Wahrscheinlichkeit eine postoperative Verbesserung [280, 281, 286-290].

\subsubsection{Core Outcome Sets (COS)}

Ein interessanter Ansatz, Studienergebnisse zur Effektivität verschiedener Therapien besser vergleichbar zu machen, ist die Entwicklung von sog. Core Outcome Sets (COS). Hier sollen die in einer Studie mindestens zu bestimmenden und später zu veröffentlichenden Messwerte für Ergebnisse festgelegt werden. Die WHO und die Cochrane Group befürworten den Einsatz entsprechender Sets. Die COMET-Initiative (Core Outcome Measures in Effectiveness Trials) hat Leitlinien zur Erstellung dieser Sets mit einer Konsensfindung nach der Delphi-Methode entwickelt [291, 292]. COS sollen die Heterogenität von Ergebnissen reduzieren und somit die Vergleichbarkeit von Studien verbessern und zudem einen Reporting bias (selektives Berichten von Endpunkten) für insbesondere negative Ergebnisse verhindern.

Für die CRS wurde ein Set mit 15 Punkten entwickelt [293]:

- Schwere, Dauer, Häufigkeit der Symptome

- Dauer des Therapieeffektes 
- Rhinorrhoe

- Nasenatmungsbehinderung

- Riechvermögen

- Krankheitsspezifische Lebensqualität

- Endoskopischer Befund

- Kontrolle der Erkrankung

- Notwendigkeit chirurgischer Eingriffe

- Fähigkeit normale Aktivitäten auszuführen

- Compliance hinsichtlich der Therapie

- Akzeptanz der Therapie

- Nebenwirkungen/Komplikationen der Behandlung

Eine Modifikation des SNOT-22, der über die Zeit wiederholt angewendet würde, könnte die Bewertung der o.g. Punkte mit einem einzigen PROM zusätzlich zur Bewertung des endoskopischen Befundes mittels des Lund-Kennedy-Scores ermöglichen [293].

\subsubsection{Effizienz}

Für die Betrachtung der Effizienz der Nasennebenhöhlenchirurgie soll zum einen die Effektivität hinsichtlich der krankheitsspezifischen Lebensqualität und zum anderen die Kosteneffizienz im Vergleich zur fortgeführten medikamentösen Therapie betrachtet werden.

Sowohl die Nasennebenhöhlenchirurgie als auch die fortgeführte medikamentöse Therapie haben sich bei Patienten mit persistierenden Beschwerden nach adäquater medikamentöser Therapie als wirksam erwiesen. Um limitierte Ressourcen des Gesundheitssystems effizient zu nutzen, ist es wichtig zu definieren, welche Patientengruppen am ehesten von der jeweiligen Therapie profitieren könnten.

Frühere systematische Reviews scheiterten an der Qualität der vorhandenen Studien. Zusätzlich war aufgrund der fehlenden Definition einer adäquaten medikamentösen Therapie zur Definition therapierefraktärer Fälle die Studienlage sehr inhomogen und z. T. wurden auch Patienten ohne vorherige medikamentöse Therapie eingeschlossen. Es zeigten sich keine Unterschiede zwischen medikamentöser Therapie und Nasennebenhöhlenchirurgie. Aufgrund der schlechten Datenlage konnten jedoch nur schwache Empfehlungen ausgesprochen werden. [294-297].

Die Schwierigkeit besteht im Design einer Studie mit entsprechend hoher Qualität, da sich aus ethischen Gründen das Vortäuschen einer Operation zur Verblindung verbietet und eine Randomisierung von Patienten in eine der beiden Therapiegruppen und so ein evtl. Vorenthalten bzw. Hinauszögern einer wahrscheinlich erfolgreichen Therapieform ebenfalls ethisch fragwürdig ist, zumal sich, wie im Abschnitt Zeitpunkt der Operation dargestellt, zeigt, dass eine frühzeitige Operation das postoperative Ergebnis verbessern kann. Bei nichtrandomisierten Studien ergibt sich jedoch die Gefahr eines Selektionsbias. Ein weiteres Problem chirurgischer Studien besteht darin, dass eine vollständige Standardisierung eines chirurgischen Eingriffs nicht möglich ist. Die chirurgischen Fähigkeiten und die Erfahrung der Chirurgen können das Ergebnis beeinflussen [298].

In neueren prospektiven Studien zeigt sich bei Patienten mit starker Einschränkung der Lebensqualität eine signifikante Verbesserung nach chirurgischer Therapie im Vergleich zu fortgeführter medikamentöser Therapie. Diese Verbesserungen betreffen den Gesamtpunktwert des SNOT-22, sowie die Untergruppen rhinologische und psychologische Beeinträchtigung und schlafbezogene Beschwerden und den Rhinosinusitis Disability Index (RSDI), Chronic Sinusitis Survey (CSS) und Short Form-6D (SF-6D). Es zeigen sich zudem Verbesserungen im endoskopischen Befund und eine Reduktion der Krankheitstage. Bei fortgeführter medikamentöser Therapie kommt es zum Teil zu einer Verschlechterung der Lebensqualität, des endoskopischen Befundes und zu einer Zunahme der Krankheitstage [50, 51, 220, 299-302]. Konservativ behandelte Patienten erreichen zwar im Durchschnitt eine signifikante Verbesserung des Gesamtpunktwertes des SNOT-22, jedoch liegt die Verbesserung unter der minimalen klinisch relevanten Differenz [21]. Bei Patienten mit geringer Einschränkung der Lebensqualität zeigen sich stabile Werte und z.T. eine signifikante Verbesserung unter medikamentöser Therapie [221, 299, 301]. Eine Metaanalyse bestätigt diese Ergebnisse mit Ausnahme der Reduktion der Krankheitstage [22].

Die Nasennebenhöhlenchirurgie ist zudem effektiver in der Kontrolle der Kardinalsymptome der CRS (zähe Rhinorrhoe, Nasenatmungsbehinderung, Gesichtsschmerz/druck) als die fortgeführte medikamentöse Therapie [49].

Zusätzlich zu objektiven Befunden und dem Ausmaß der Einschränkung der Lebensqualität könnte in Zukunft auch die Bestimmung spezifischer Biomarker und somit die Endotypisierung, aufgrund des unterschiedlichen Ansprechens auf die jeweiligen Therapiearten, in der Therapieentscheidung zwischen fortgesetzter medikamentöser Therapie und Nasennebenhöhlenchirurgie eine Rolle spielen [111,303].

In verschiedenen Kosten-Nutzwert-Analysen hat sich die chirurgische gegenüber der medikamentösen Therapie als kosteneffizienter erwiesen, sowohl bei Betrachtung der CRS insgesamt, als auch bei Betrachtung von Subgruppen einer CRSsNP und CRSwNP, als auch von Patienten mit Asthma bronchiale. In verschiedenen amerikanischen Studien der Versorgungsforschung wurden Patientenkollektive mit CRS, CRSsNP, CRSwNP und gleichzeitig bestehendem Asthma bronchiale, die nach Versagen der medikamentösen Therapie entweder weiter medikamentös behandelt wurden oder sich einer Operation der Nasennebenhöhlen unterzogen, analysiert [25, 304, 305]. Berechnet wurden zum einen die sog. QALYs (quality adjusted life year = qualitätskorrigiertes Lebensjahr) und zum anderen das inkrementelle Kosten-Effektivitäts-Verhältnis (incremental cost effectiveness ratio = ICER). Das inkrementelle KostenEffektivitäts-Verhältnis berechnet das Verhältnis zwischen der Kostendifferenz zweier Behandlungsstrategien und der unterschiedlichen Wirksamkeit dieser Strategien, d.h. die zusätzlichen Kosten, die mit dem zusätzlichen Nutzen einer Behandlung assoziiert sind. Die genaue Beschreibung des Vorgehens ist den entsprechenden Artikeln zu entnehmen [25, 304]. Ein QALY ist eine Kennzahl für die Bewertung eines Lebensjahres in Relation zur Gesundheit. Ein QALY von 1 bedeutet ein Jahr in voller Gesundheit, während ein QALY von 0 einem Versterben entspricht. Das Konzept des QALYs ist aufgrund methodischer und ethischer Kritikpunkte nicht unumstritten. Kritisiert wird u.a., dass nicht berücksichtigt wird, dass eine geringe Verbesserung des Gesundheitszustandes umso besser bewertet wird, je schlechter der Allgemeinzustand vorher war. Zudem besteht der Vorwurf einer Diskriminierung von Kranken, Behinderten und älteren Menschen. Da in die Berechnung des QALY die Lebenserwartung miteinbezogen wird, können ältere im Vergleich zu jungen Menschen nur wenige QALYs hinzugewinnen. 
Bei Betrachtung eines CRS-Kollektivs ohne Subgruppen ergeben sich über eine Behandlungsdauer von 30 Jahren Gesamtkosten für die Nasennebenhöhlenchirurgie-Strategie von 48838,38 USD mit insgesamt 20,50 QALYs und bei medikamentöser Therapie 28948,98 USD mit insgesamt 17,13 QALYs. Das inkrementelle Kosten-Effektivitäts-Verhältnis liegt bei 5901,90 USD pro QALY für die Nasennebenhöhlenchirurgie im Verhältnis zur medikamentösen Therapie [25].

In einer weiteren Studie mit Unterscheidung zwischen CRSsNP und CRSwNP ergibt sich ein ähnliches Bild für beide Subgruppen (ICER: CRSwNP 5687,41 USD/QUALY, CRSsNP 5405,44 USD/QALY) [304]. Die höheren Kosten der chirurgischen Therapie einer CRSwNP im Vergleich zur CRSsNP werden durch den höheren Gewinn an Lebensqualität, der auch in anderen Studien beschrieben wurde, aufgefangen [123].

Asthma bronchiale ist eine häufige Komorbidität der CRSwNP [306]. In einer Analyse von CRSwNP-Patienten mit und ohne Asthma zeigt sich ebenfalls in beiden Gruppen eine höhere Kosteneffizienz des chirurgischen Vorgehens im Vergleich zur fortgeführten medikamentösen Therapie bei therapierefraktärer CRS [305].

Diese Art der Kosten-Nutzwert-Analyse hat die Einschränkung, dass es sich um theoretische Modelle handelt, die zwar z. T. mit Patientendaten, aber z. T. auch nur aufgrund von Daten eingeschränkter Qualität aus Studien berechnet werden. Außerdem fehlen entsprechende Studien aus dem deutschsprachigen Raum, die das deutsche Gesundheitssystem und dessen Kosten berücksichtigen.

Eine medizinische Entscheidung aufgrund monetärer Aspekte ruft berechtigterweise großes Unbehagen hervor. Jenseits der Kosten-Nutzwert-Analysen muss berücksichtigt werden, dass die Entscheidung zur Operation nicht unter monetären Gesichtspunkten getroffen werden darf, sondern individualisiert auf den jeweiligen Patienten abgestimmt werden muss und als patientenzentrierte Entscheidung, die Ziele und Präferenzen des Patienten berücksichtigen soll. Bei der Betrachtung der Effizienz im Qualitätsmanagement geht es um die Vermeidung von Verschwendung. Knappe Mittel sollen aus ökonomischer Sicht so eingesetzt werden, dass der größtmögliche Nutzen „produziert“ wird. Es sollen rationale Entscheidungen über eine optimale Ressourcennutzung ermöglicht werden. Es bleibt somit nicht aus, dass man sich auch als Arzt mit der Kosteneffizienz auseinandersetzt.

\section{Rezidivierende akute Rhinosinusitis}

Die rezidivierende akute Rhinosinusitis (RARS) ist eine zu den chronischen Rhinosinusitiden zählende Erkrankung. Die jährliche Prävalenz wird in der Literatur mit 0,035\% angegeben. In einer US-amerikanischen Studie ergaben sich jährliche direkte Kosten von durchschnittlich 1091 USD pro Patient [307].

\subsection{Diagnose}

Nach den Clinical practice guidelines der American Academy of Otolaryngology-Head and Neck Surgery Foundation erfordert die Diagnose der RARS mehr als 4 Episoden einer akuten bakteriellen Sinusitis pro Jahr mit symptomfreien Intervallen zwischen den Episoden. Jede Episode muss die folgenden Kriterien erfüllen: Symptome oder Zeichen einer akuten Rhinosinusitis ohne Besserung innerhalb von 10 oder mehr Tagen nach dem Auftreten von Symptomen eines obe- ren Atemwegsinfektes oder Verschlechterung innerhalb von 10 Tagen nach initialer klinischer Besserung [30]. Problematisch bei der Diagnosestellung ist die intermittierende Symptomatik mit Normbefunden im entzündungsfreien Intervall und die häufig schwierige Unterscheidung zwischen viraler und bakterieller Infektion aufgrund der anamnestischen Angaben des Patienten [308-310].

\subsection{Medikamentöse Therapie}

Die primäre Therapie der RARS besteht in einer medikamentösen Therapie. In der Regel werden eine kurzzeitige antibiotische Therapie während akuter Phasen, sowie eine topische Steroidtherapie und Nasenspülungen empfohlen. In einem systematischen Review konnte kein Artikel, der die Effektivität einer kurzzeitigen antibiotischen Therapie bei Patienten mit RARS gegenüber Placebo prüft, identifiziert werden [311]. In einer identischen Arbeit zur topischen Steroidtherapie finden sich 3 Studien, die unterschiedliche topische Steroide mit Placebo zusätzlich zu einer antibiotischen Therapie vergleichen. Es kommt zu einer rascheren Symptombesserung, einer Verbesserung der klinischen Erfolgsrate und einer Reduktion der Rezidivrate bei der Anwendung von topischen Steroiden. Eine Studie zeigt keinen Unterschied zu Placebo [312]. Der Vorteil einer Monotherapie mit Steroiden bleibt unklar. Zudem lässt die Verwendung unterschiedlicher Steroide, Dosierungen und die unterschiedliche Anwendungsdauer keine eindeutige Empfehlung zu [313]. Bei einem Vergleich zwischen medikamentöser und chirurgischer Therapie zeigt sich eine signifikante Verbesserung der Lebensqualität im SNOT-22 12 Monate postoperativ unter medikamentöser Therapie akuter Episoden mit Antibiotika und topischem Steroid sowie Nasenspülungen [308].

\subsection{Patientenselektion und patientenzentrierte Entscheidungsfindung}

Kriterien, die in der Entscheidungsfindung zwischen konservativer und chirurgischer Therapie eine Rolle spielen, sind die Häufigkeit und Schwere der akuten Episoden und die damit einhergehende Einschränkung der Lebensqualität und Produktivität. Entsprechend der im Abschnitt 3.1.2 beschriebenen RAND/UCLA-Methode wurden in einem Expertenkonsens amerikanischer und kanadischer Kollegen Kriterien zur Patientenselektion für die endonasale endoskopische Nasennebenhöhlenchirurgie als Therapie der RARS formuliert:

- mindestens eine Episode einer akuten bakteriellen Rhinosinusitis, die durch einen objektiven Befund im CT oder der nasalen Endoskopie bestätigt wurde

- patientenzentrierte Entscheidungsfindung mit Diskussion der Risiken und des Nutzens der Nasennebenhöhlenchirurgie unter Berücksichtigung des erwarteten Ergebnisses und der Behandlungsalternativen

- frustraner Therapieversuch mit topischem Steroid oder signifikante Reduktion der alltäglichen Produktivität aufgrund der RARS

Es wird betont, dass diese Kriterien die minimalen Anforderungen darstellen, um die Nasennebenhöhlenchirurgie als Behandlungsoption in Betracht zu ziehen. Kritisch zu sehen ist der Nachweis von objektiven Befunden im CT, da dies im Rahmen einer komplikationslosen akuten bakteriellen Rhinosinusitis in der Regel nicht indiziert ist, um die Strahlenbelastung gering zu halten. Der 
endoskopische Befund mit putrider Sekretion im mittleren Nasengang scheint sinnvoller. Die Objektivierung der anamnestischen Angaben des Patienten wird von den Mitgliedern des Expertenpanels zur Abgrenzung von Differenzialdiagnosen, wie Migräne oder Gesichtsschmerz, als wichtig erachtet. Die Evidenzlage zur Therapie der RARS ist eingeschränkt, sodass die o.g. Empfehlungen im Wesentlichen auf Expertenmeinungen und der Interpretation der vorhandenen Literatur beruhen [314]. Bei der Diskussion der Risiken sollten auch die möglichen Komplikationen einer langfristigen wiederholten antibiotischen Therapie, wie die Entwicklung von Resistenzen und gastrointestinale Nebenwirkungen, berücksichtigt werden.

Ähnliche Kriterien zur Indikationsstellung finden sich in weiteren Studien [309, 315-316]

\subsection{Postoperatives Ergebnis}

Da die Pathogenese der RARS unklar ist, könnte das Ansprechen auf die chirurgische Therapie sehr unterschiedlich ausfallen. Bei Patienten mit RARS wird im Rahmen einer Operation am häufigsten eine Kieferhöhlenoperation und vordere Ethmoidektomie durchgeführt [308, 317, 318]. Auch die Ballon-Sinuplastik zeigte positive Effekte auf die Lebensqualität und die Anzahl der Infektionen [319]. Nach Nasennebenhöhlenchirurgie kommt es zu einer signifikanten Reduktion der Anzahl der Infektionen. Zur Änderung des postoperativen Antibiotikaverbrauchs zeigen sich unterschiedliche Ergebnisse. In einigen Studien kam es zu einer Abnahme der Gesamtdauer der Antibiotikaeinnahme und der Anzahl der Antibiotikagaben [317, 320], wohingegen andere keinen Unterschied zeigten [315].

Aktuell existieren keine Studien, die die Ergebnisse nach unterschiedlich ausgedehnter Operation vergleichen. Es erscheint sinnvoll, die bei einer rezidivierenden akuten Entzündung primär klinisch betroffenen Nasennebenhöhlen zu operieren. Es stellt sich die Frage, ob ein zunächst konservativeres und bei Erfolglosigkeit ausgedehnteres Vorgehen in einer Abwägung von Risiko und Nutzen das beste Vorgehen darstellt, v.a. wenn nicht nur die Kieferhöhle betroffen ist. Hierzu sind entsprechende Studien notwendig.

\subsubsection{Allgemeine Messinstrumente der gesundheitsbezoge- nen Lebensqualität}

Patienten mit RARS geben präoperativ eine mit CRSsNP-Patienten vergleichbare Einschränkung des Gesundheitszustandes im SF-6D an. Die präoperativen Werte zeigen keine signifikante Korrelation mit dem CT-Score nach Lund-Mackay und dem Endoskopie-Score nach Lund-Kennedy. Postoperativ kommt es zu einer signifikanten Verbesserung des Gesundheitszustandes, gemessen mit dem SF-6D. Dieser lag bei durchschnittlich 0,08. Zum Vergleich erreichen Patienten mit OSAS 1 Jahr nach CPAP-Therapie eine Verbesserung des Gesundheitszustandes gemessen mit dem SF-6D von 0,10 und Patienten mit Osteoarthritis eine Verbesserung von ebenfalls 0,10 nach Hüft-TEP [321, 322] Die Werte zeigten keinen signifikanten Unterschied zwischen Patienten mit RARS und CRSsNP. Im Durchschnitt erreichten alle Patienten die minimale klinisch relevante Differenz von $>0,03$ [318].

\subsubsection{Spezifische Messinstrumente der gesundheitsbezoge- nen Lebensqualität}

Die Einschränkung der Lebensqualität von Patienten mit RARS entspricht der von Patienten mit CRSsNP in verschiedenen Lebensqua-
litäts-Messinstrumenten, wie dem RSDI, dem RSI, dem CSS, dem SNOT-20 und dem SNOT-22 bei in der Regel geringeren objektiven Befunden im entzündungsfreien Intervall (CT, Endoskopie) [308, 317, 318, 323, 324]. Patienten mit RARS zeigen statistisch höhere Werte für oropharyngeale und systemische Symptome und zudem mehr Krankheitstage und häufigere Antibiotikagaben als Patienten mit CRS.

Die chirurgische Therapie führt im Vergleich mit einer medikamentösen Therapie zu einer größeren Verbesserung der krankheitsspezifischen Lebensqualität, gemessen durch den SNOT-22 [308]. Patienten unter zunächst medikamentöser Therapie entscheiden sich im Verlauf nicht selten für ein chirurgisches Vorgehen (33-76\%) [308, 319]. Vor einem Therapiewechsel konnte eine Verschlechterung der Lebensqualität nachgewiesen werden [308]. Auch bei diesen Patienten kommt es postoperativ zu einer signifikanten Verbesserung der Lebensqualität.

Mehrere Studien konnten eine signifikante Verbesserung der krankheitsspezifischen Lebensqualität durch die Nasennebenhöhlenchirurgie, gemessen anhand des SNOT-20, SNOT-22, RSI, RSDI und CSS feststellen [308, 309, 315, 317, 324]. Es zeigten sich stabile Ergebnisse über bis zu 19 Monate [309, 315].

Die Problematik in der Lebensqualitätsmessung bei Patienten mit RARS liegt darin, dass die vorhandenen Messinstrumente nicht für Erkrankungen mit periodischer Symptomatik ausgelegt sind und dass akute Episoden retrospektiv durch den Patienten beurteilt werden sollen, was zu entsprechenden Ungenauigkeiten in den Angaben führen kann. Beispielsweise erfragt der SNOT-22 die Symptomatik in den vorangegangenen 2 Wochen, was entweder eine symptomfreie Periode oder eine akute Entzündung betreffen kann.

\subsubsection{Produktivität}

Die Anzahl von Fehltagen pro Jahr wird mit durchschnittlich 4,2-7,1 angegeben [307, 315]. Bezieht man die Tage reduzierter Produktivität am Arbeitsplatz (Präsentismus) mit ein, so geben Patienten mit RARS durchschnittlich 12,6 Tage in 3 Monaten an. Präoperativ zeigt sich kein signifikanter Unterschied zu Patienten mit CRSsNP [317]. Nach Nasennebenhöhlenchirurgie kommt es zu einer signifikanten Reduktion der Fehltage [315]. Unter Einbeziehung des Präsentismus zeigt sich eine $>50 \%$ ige Reduktion der Tage mit verlorener oder reduzierter Produktivität [317].

Um zu definieren, wann ein chirurgisches Vorgehen einer medikamentösen Therapie vorzuziehen ist, wurden wie auch bei der CRS, verschiedene Modelle herangezogen. In einem makroökonomischen Ansatz wurde in einer produktivitätsbasierten Analyse mit einem Modellvergleich zwischen medikamentöser und chirurgischer Therapie ein Schwellenwert von 6 Episoden einer RARS pro Jahr für die Erwägung eines chirurgischen Vorgehens ermittelt [325]. Die Entscheidung auf Patientenebene wird jedoch auch durch finanzielle Faktoren, wie Eigenanteile an medizinischen Kosten und Gehaltseinbußen und die Lebensqualität beeinflusst. Eine US-amerikanische Studie ermittelte den Break-even-Punkt, an dem eine chirurgische Therapie einer medikamentösen Therapie vorzuziehen ist, aus der Perspektive der Kosten für den Patienten in Relation zur Änderung der Lebensqualität [326]. Ermittelt wurde die Anzahl der Infektionen pro Jahr, ab der ein chirurgisches Vorgehen weniger Kosten pro Einheit Lebensqualität verursacht als wiederholte medikamentöse Therapieversuche. Es ergab sich ein Grenzwert von 5 Episoden pro Jahr 
[326]. Hierbei müssen die Unterschiede zwischen den Gesundheitssystemen in den USA und Deutschland berücksichtigt werden, so dass die Ergebnisse nicht direkt übertragen werden können.

\section{Danksagung}

Ich möchte mich bei Frau Karin Focke für die Unterstützung bei der Beschaffung der Literatur und Frau Christine Isele für die Hilfe bei der Umfrage für dieses Referat bedanken. Mein ganz herzlicher Dank gilt Herrn Prof. Rainer Weber, Herrn Prof. Werner Hosemann und Herrn Prof. Andreas Knopf für die kritische Durchsicht des Manuskriptes und die bereichernde Diskussion.

Interessenkonflikt

Die Autoren geben an, dass kein Interessenkonflikt besteht.

\section{Literatur}

[1] Rudmik L, Smith TL, Schlosser RJ et al. Productivity costs in patients with refractory chronic rhinosinusitis. Laryngoscope 2014; 124 : 2007-2012

[2] Gliklich RE, Metson R. The health impact of chronic sinusitis in patients seeking otolaryngologic care. Otolaryngol Head Neck Surg 1995; 113: 104-109

[3] Hastan D, Fokkens W], Bachert C et al. Chronic rhinosinusitis in Europe - an underestimated disease. A GA(2)LEN study. Allergy 2011; 66: 1216-1223

[4] Fleming-Dutra KE, Hersh AL, Shapiro DJ et al. Prevalence of Inappropriate Antibiotic Prescriptions Among US Ambulatory Care Visits, 2010-2011. JAMA 2016; 315: 1864-1873

[5] Rudmik L, Mattos JL, Stokken JK et al. Rhinology-specific priority setting for quality improvement: a modified Delphi study from the Quality Improvement Committee of the American Rhinologic Society. Int Forum Allergy Rhinol 2017; 7: 937-944

[6] Stuck BA, Beule A, Jobst D et al. Guideline for "rhinosinusitis"-long version : S2k guideline of the German College of General Practitioners and Family Physicians and the German Society for Oto-RhinoLaryngology, Head and Neck Surgery. HNO 2018; 66: 38-74

[7] Rudmik L, Holy CE, Smith TL. Geographic variation of endoscopic sinus surgery in the United States. Laryngoscope 2015; 125: 1772-1778

[8] Rudmik L, Bird C, Dean S et al. Geographic Variation of Endoscopic Sinus Surgery in Canada: An Alberta-Based Small Area Variation Analysis. Otolaryngol Head Neck Surg 2015; 153: 865-874

[9] Venkatraman G, Likosky DS, Morrison D et al. Small area variation in endoscopic sinus surgery rates among the Medicare population. Arch Otolaryngol Head Neck Surg 2011; 137: 253-257

[10] Svider PF, Sekhsaria V, Cohen DS et al. Geographic and temporal trends in frontal sinus surgery. Int Forum Allergy Rhinol 2015; 5: 46-54

[11] Fokkens W], Lund V], Mullol J et al. EPOS 2012: European position paper on rhinosinusitis and nasal polyps 2012. A summary for otorhinolaryngologists. Rhinology 2012; 50: 1-12

[12] Weber RK, Hosemann W. Comprehensive review on endonasal endoscopic sinus surgery. GMS Curr Top Otorhinolaryngol Head Neck Surg 2015; 14: Doc08
[13] Chester AC, Antisdel JL, Sindwani R. Symptom-specific outcomes of endoscopic sinus surgery: a systematic review. Otolaryngol Head Neck Surg 2009; 140: 633-639

[14] Dalziel K, Stein K, Round A et al. Systematic review of endoscopic sinus surgery for nasal polyps. Health Technol Assess 2003; 7: iii $1-159$

[15] Georgalas C, Cornet M, Adriaensen G et al. Evidence-based surgery for chronic rhinosinusitis with and without nasal polyps. Curr Allergy Asthma Rep 2014; 14: 427

[16] Smith TL, Batra PS, Seiden AM et al. Evidence supporting endoscopic sinus surgery in the management of adult chronic rhinosinusitis: a systematic review. Am J Rhinol 2005; 19: 537-543

[17] Giger R, Dulguerov P, Quinodoz D et al. Chronic panrhinosinusitis without nasal polyps: long-term outcome after functional endoscopic sinus surgery. Otolaryngol Head Neck Surg 2004; 131: 534-541

[18] Toros SZ, Bolukbasi S, Naiboglu B et al. Comparative outcomes of endoscopic sinus surgery in patients with chronic sinusitis and nasal polyps. Eur Arch Otorhinolaryngol 2007; 264: 1003-1008

[19] Gliklich RE, Metson R. Effect of sinus surgery on quality of life. Otolaryngol Head Neck Surg 1997; 117: 12-17

[20] Sukato DC, Abramowitz JM, Boruk M et al. Endoscopic Sinus Surgery Improves Sleep Quality in Chronic Rhinosinusitis: A Systematic Review and Meta-analysis. Otolaryngol Head Neck Surg 2018; 158: 249-256

[21] Beswick DM, Mace JC, Rudmik L et al. Productivity changes following medical and surgical treatment of chronic rhinosinusitis by symptom domain. Int Forum Allergy Rhinol 2018; 8: 1395-1405

[22] Patel ZM, Thamboo A, Rudmik L et al. Surgical therapy vs continued medical therapy for medically refractory chronic rhinosinusitis: a systematic review and meta-analysis. Int Forum Allergy Rhinol 2017; 7: $119-127$

[23] Vashishta R, Soler ZM, Nguyen SA et al. A systematic review and meta-analysis of asthma outcomes following endoscopic sinus surgery for chronic rhinosinusitis. Int Forum Allergy Rhinol 2013; 3: 788-794

[24] Hopkins C, Browne JP, Slack R et al. The national comparative audit of surgery for nasal polyposis and chronic rhinosinusitis. Clin Otolaryngol 2006; 31: 390-398

[25] Rudmik L, Soler ZM, Mace JC et al. Economic evaluation of endoscopic sinus surgery versus continued medical therapy for refractory chronic rhinosinusitis. Laryngoscope 2015; 125: 25-32

[26] Donabedian A. Evaluating the quality of medical care. Milbank Mem Fund Q 1966; 44: Suppl 166-206

[27] Mattos JL, Soler ZM, Rudmik L et al. A framework for quality measurement in the presurgical care of chronic rhinosinusitis: a review from the Quality Improvement Committee of the American Rhinologic Society. Int Forum Allergy Rhinol 2018; 8: 1380-1388

[28] Cottrell ], Yip J, Chan Y et al. Quality indicators for the diagnosis and management of chronic rhinosinusitis. Int Forum Allergy Rhinol 2018; 8: 1369-1379

[29] Orlandi RR, Kingdom TT, Hwang PH et al. International Consensus Statement on Allergy and Rhinology: Rhinosinusitis. Int Forum Allergy Rhinol 2016; 6 (Suppl 1): S22-S209

[30] Rosenfeld RM, Piccirillo JF, Chandrasekhar SS et al. Clinical practice guideline (update): adult sinusitis. Otolaryngol Head Neck Surg 2015; 152: $51-S 39$

[31] Bhattacharyya N. Clinical and symptom criteria for the accurate diagnosis of chronic rhinosinusitis. Laryngoscope 2006; 116: 1-22

[32] Kalish L, Snidvongs K, Sivasubramaniam R et al. Topical steroids for nasal polyps. Cochrane Database Syst Rev 2012; 12: CD006549 
[33] Rudmik L, Soler ZM, Hopkins C et al. Defining appropriateness criteria for endoscopic sinus surgery during management of uncomplicated adult chronic rhinosinusitis: a RAND/UCLA appropriateness study. Rhinology 2016; 54: 117-128

[34] Hopkins C, Andrews P, Holy CE. Does time to endoscopic sinus surgery impact outcomes in chronic rhinosinusitis? Retrospective analysis using the UK clinical practice research data. Rhinology 2015; 53: $18-24$

[35] Hopkins C, Rudmik L, Lund VJ. The predictive value of the preoperative Sinonasal Outcome Test-22 score in patients undergoing endoscopic sinus surgery for chronic rhinosinusitis. Laryngoscope 2015; 125: 1779-1784

[36] Benninger MS, Sindwani R, Holy CE et al. Early versus delayed endoscopic sinus surgery in patients with chronic rhinosinusitis: impact on health care utilization. Otolaryngol Head Neck Surg 2015; 152: 546-552

[37] Bradley DT, Kountakis SE. Correlation between computed tomography scores and symptomatic improvement after endoscopic sinus surgery. Laryngoscope 2005; 115: 466-469

[38] Ryan WR, Ramachandra T, Hwang PH. Correlations between symptoms, nasal endoscopy, and in-office computed tomography in post-surgical chronic rhinosinusitis patients. Laryngoscope 2011; 121: 674-678

[39] Mace JC, Michael YL, Carlson NE et al. Correlations between endoscopy score and quality of life changes after sinus surgery. Arch Otolaryngol Head Neck Surg 2010; 136: 340-346

[40] Stewart MG, Sicard MW, Piccirillo JF et al. Severity staging in chronic sinusitis: are CT scan findings related to patient symptoms? Am J Rhinol 1999; 13: 161-167

[41] Wabnitz DA, Nair S, Wormald PJ. Correlation between preoperative symptom scores, quality-of-life questionnaires, and staging with computed tomography in patients with chronic rhinosinusitis. Am J Rhinol 2005; 19: 91-96

[42] Gupta D, Gulati A, Singh I et al. Endoscopic, radiological, and symptom correlation of olfactory dysfunction in pre- and postsurgical patients of chronic rhinosinusitis. Chem Senses 2014; 39: 705-710

[43] Bhattacharyya T, Piccirillo J, Wippold F] 2nd. Relationship between patient-based descriptions of sinusitis and paranasal sinus computed tomographic findings. Arch Otolaryngol Head Neck Surg 1997; 123: 1189-1192

[44] Smith TL, Rhee JS, Loehrl TA et al. Objective testing and quality-of-life evaluation in surgical candidates with chronic rhinosinusitis. Am J Rhinol 2003; 17: 351-356

[45] Beswick DM, Mace JC, Soler ZM et al. Appropriateness criteria predict outcomes for sinus surgery and may aid in future patient selection. Laryngoscope 2018; 128: 2448-2454

[46] Thamboo A, Rathor A, Borchard NA et al. Precision medicine: why surgeons deviate from "appropriateness criteria" in the management of chronic rhinosinusitis and effects on outcomes. Int Forum Allergy Rhinol 2018; 8: 1389-1394

[47] Soler ZM, Rudmik L, Hwang PH et al. Patient-centered decision making in the treatment of chronic rhinosinusitis. Laryngoscope 2013; 123: 2341-2346

[48] Orb Q, Mace JC, DeConde AS et al. Patients electing medical vs surgical treatment: emotional domain of the Rhinosinusitis Disability Index associates with treatment selection. Int Forum Allergy Rhinol 2016; 6: 315-321

[49] Rudmik L, Soler ZM, Mace JC et al. Using preoperative SNOT-22 score to inform patient decision for Endoscopic sinus surgery. Laryngoscope 2015 ; 125 : 1517-1522
[50] DeConde AS, Mace JC, Ramakrishnan VR et al. Analysis of factors associated with electing endoscopic sinus surgery. Laryngoscope 2018; 128: 304-310

[51] DeConde AS, Mace JC, Bodner T et al. SNOT-22 quality of life domains differentially predict treatment modality selection in chronic rhinosinusitis. Int Forum Allergy Rhinol 2014; 4: 972-979

[52] Newton E, Janjua A, Lai E et al. The impact of surgical wait time on patient reported outcomes in sinus surgery for chronic rhinosinusitis. Int Forum Allergy Rhinol 2017; 7: 1156-1161

[53] Sahlstrand-Johnson P, Hopkins C, Ohlsson B et al. The effect of endoscopic sinus surgery on quality of life and absenteeism in patients with chronic rhinosinuitis - a multi-centre study. Rhinology 2017; 55: 251-261

[54] Alt JA, Orlandi RR, Mace JC et al. Does Delaying Endoscopic Sinus Surgery Adversely Impact Quality-of-Life Outcomes? Laryngoscope 2019; 129: 303-311

[55] Benninger MS, Sindwani R, Holy CE et al. Impact of medically recalcitrant chronic rhinosinusitis on incidence of asthma. Int Forum Allergy Rhinol 2016; 6: 124-129

[56] Unsal AA, Gregory N, Rosenstein K. Current opinions in office-based rhinology. Curr Opin Otolaryngol Head Neck Surg 2018; 26: 8-12

[57] Lee JT, DelGaudio J, Orlandi RR. Practice Patterns in Office-Based Rhinology: Survey of the American Rhinologic Society. Am J Rhinol Allergy 2019; 33: 26-35

[58] Hopkins C, Browne J, Slack R et al. Variation in day-case nasal surgery - why cannot we improve our day-case rates? Clin Otolaryngol 2007; 32: $12-18$

[59] Georgalas C, Obholzer R, Martinez-Devesa P et al. Day-case septoplasty and unexpected re-admissions at a dedicated day-case unit: a 4-year audit. Ann R Coll Surg Engl 2006; 88: 202-206

[60] Bhattacharyya N. Unplanned revisits and readmissions after ambulatory sinonasal surgery. Laryngoscope 2014; 124: 1983-1987

[61] Bhattacharyya N. Ambulatory sinus and nasal surgery in the United States: demographics and perioperative outcomes. Laryngoscope 2010; 120: 635-638

[62] Bajaj Y, Sethi N, Carr S et al. Endoscopic sinus surgery as day-case procedure. J Laryngol Otol 2009; 123: 619-622

[63] Ganesan S, Prior AJ, Rubin JS. Unexpected overnight admissions following day-case surgery: an analysis of a dedicated ENT day care unit. Ann R Coll Surg Engl 2000; 82: 327-330

[64] Scott JR, Sowerby L], Rotenberg BW. Office-based rhinologic surgery: A modern experience with operative techniques under local anesthetic. Am J Rhinol Allergy 2017; 31: 135-138

[65] Radvansky BM, Husain Q, Cherla DV et al. In-office vasovagal response after rhinologic manipulation. Int Forum Allergy Rhinol 2013; 3: 510-514

[66] Haynes AB, Weiser TG, Berry WR et al. A surgical safety checklist to reduce morbidity and mortality in a global population. $N$ Engl J Med 2009; 360: 491-499

[67] van Klei WA, Hoff RG, van Aarnhem EE et al. Effects of the introduction of the WHO "Surgical Safety Checklist" on in-hospital mortality: a cohort study. Ann Surg 2012; 255: 44-49

[68] Buzink SN, van Lier L, de Hingh IH et al. Risk-sensitive events during laparoscopic cholecystectomy: the influence of the integrated operating room and a preoperative checklist tool. Surg Endosc 2010; 24: 1990-1995

[69] Weiser TG, Haynes AB, Lashoher A et al. Perspectives in quality: designing the WHO Surgical Safety Checklist. Int J Qual Health Care 2010; 22 : 365-370

[70] Soler ZM, Smith TL. Endoscopic sinus surgery checklist. Laryngoscope 2012; 122: 137-139 
[71] Soler ZM, Poetker DA, Rudmik L et al. Multi-institutional evaluation of a sinus surgery checklist. Laryngoscope 2012; 122: 2132-2136

[72] O'Brien WT Sr., Hamelin S, Weitzel EK. The Preoperative Sinus CT: Avoiding a "CLOSE" Call with Surgical Complications. Radiology 2016; 281: 10-21

[73] Simmen D, Schuknecht B. Computerized tomography of paranasal sinuses--a preoperative check list. Laryngorhinootologie 1997; 76: 8-13

[74] Martinez Del Pero M, Philpott C. A useful tool - systematic checklist for evaluating sinus scans. Clin Otolaryngol 2012; 37: 82-84

[75] Yao CM, Fernandes VT, Palmer JN et al. Educational value of a preoperative CT sinus checklist: a resident's perspective. J Surg Educ 2013; 70: $585-587$

[76] Error M, Ashby S, Orlandi RR et al. Single-Blinded Prospective Implementation of a Preoperative Imaging Checklist for Endoscopic Sinus Surgery. Otolaryngol Head Neck Surg 2018; 158: 177-180

[77] Urbach DR, Govindarajan A, Saskin R et al. Introduction of surgical safety checklists in Ontario, Canada. N Engl J Med 2014; 370 : 1029-1038

[78] Lund V]. The results of inferior and middle meatal antrostomy under endoscopic control. Acta Otorhinolaryngol Belg 1993; 47: 65-71

[79] Penttila MA, Rautiainen ME, Pukander JS et al. Endoscopic versus Caldwell-Luc approach in chronic maxillary sinusitis: comparison of symptoms at one-year follow-up. Rhinology 1994; 32: 161-165

[80] Joe Jacob K, George S, Preethi S et al. A comparative study between endoscopic middle meatal antrostomy and caldwell-luc surgery in the treatment of chronic maxillary sinusitis. Indian J Otolaryngol Head Neck Surg 2011; 63: 214-219

[81] Venkatachalam VP, Jain A. Comparative evaluation of functional endoscopic sinus surgery and conventional surgery in the management of chronic sinusitis. J Indian Med Assoc 2002; 100: 78-79 $82-73$

[82] Cote M, Kalra R, Wilson T et al. Surgical fidelity: comparing the microscope and the endoscope. Acta Neurochir (Wien) 2013; 155: 2299-2303

[83] Tasman AJ, Wallner F, Kolling GH et al. Is monocular perception of depth through the rigid endoscope a disadvantage compared to binocular vision through the operating microscope in paranasal sinus surgery? Am J Rhinol 1998; 12: 87-91

[84] Tasman AJ, Wallner F, Kolling GH. How good is spatial orientation with rigid endoscopic optics? Study of micromanipulation in the nasal cavity. HNO 1996; 44: 73-77

[85] Tasman AJ, Stammberger H. Video-endoscope versus endoscope for paranasal sinus surgery: influence on stereoacuity. Am J Rhinol 1998; 12: $389-392$

[86] Tasman AJ, Feldhusen F, Kolling GH et al. Video-endoscope versus endoscope for paranasal sinus surgery: influence on visual acuity and color discrimination. Am J Rhinol 1999; 13: 7-10

[87] Albrecht T, Baumann I, Plinkert PK et al. Three-dimensional endoscopic visualization in functional endoscopic sinus surgery. Eur Arch Otorhinolaryngol 2016; 273: 3753-3758

[88] Shah RN, Leight WD, Patel MR et al. A controlled laboratory and clinical evaluation of a three-dimensional endoscope for endonasal sinus and skull base surgery. Am J Rhinol Allergy 2011; 25: 141-144

[89] Ogino-Nishimura E, Nakagawa T, Sakamoto T et al. Efficacy of three-dimensional endoscopy in endonasal surgery. Auris Nasus Larynx 2015; 42: 203-207

[90] Nassimizadeh A, Zaidi SM, Nassimizadeh M et al. Endoscopic training-is the future three-dimensional? Laryngoscope Investig Otolaryngol 2018; 3: 345-348
[91] Rampinelli V, Doglietto F, Mattavelli D et al. Two-Dimensional High Definition Versus Three-Dimensional Endoscopy in Endonasal Skull Base Surgery: A Comparative Preclinical Study. World Neurosurg 2017; 105: 223-231

[92] Re M, Massegur H, Magliulo G et al. Traditional endonasal and microscopic sinus surgery complications versus endoscopic sinus surgery complications: a meta-analysis. Eur Arch Otorhinolaryngol 2012; 269: 721-729

[93] Ramakrishnan VR, Orlandi RR, Citardi M] et al. The use of imageguided surgery in endoscopic sinus surgery: an evidence-based review with recommendations. Int Forum Allergy Rhinol 2013; 3: 236-241

[94] Casiano RR, Numa WA Jr. Efficacy of computed tomographic image - guided endoscopic sinus surgery in residency training programs. Laryngoscope 2000; 110: 1277-1282

[95] Wise SK, Harvey RJ, Goddard JC et al. Combined image guidance and intraoperative computed tomography in facilitating endoscopic orientation within and around the paranasal sinuses. Am J Rhinol 2008; 22: 635-641

[96] Theodoraki MN, Ledderose G], Becker S et al. Mental distress and effort to engage an image-guided navigation system in the surgical training of endoscopic sinus surgery: a prospective, randomised clinical trial. Eur Arch Otorhinolaryngol 2015; 272: 905-913

[97] Dixon B], Chan H, Daly MJ et al. The effect of augmented real-time image guidance on task workload during endoscopic sinus surgery. Int Forum Allergy Rhinol 2012; 2: 405-410

[98] Dalgorf DM, Sacks R, Wormald PJ et al. Image-guided surgery influences perioperative morbidity from endoscopic sinus surgery: a systematic review and meta-analysis. Otolaryngol Head Neck Surg 2013; 149: 17-29

[99] Tabaee A, Hsu AK, Shrime MG et al. Quality of life and complications following image-guided endoscopic sinus surgery. Otolaryngol Head Neck Surg 2006; 135: 76-80

[100] Sunkaraneni VS, Yeh D, Qian H et al. Computer or not? Use of image guidance during endoscopic sinus surgery for chronic rhinosinusitis at St Paul's Hospital, Vancouver, and meta-analysis. J Laryngol Otol 2013; 127: 368-377

[101] Mueller SA, Caversaccio M. Outcome of computer-assisted surgery in patients with chronic rhinosinusitis. J Laryngol Otol 2010; 124: 500-504

[102] Reardon EJ. Navigational risks associated with sinus surgery and the clinical effects of implementing a navigational system for sinus surgery. Laryngoscope 2002; 112: 1-19

[103] Fried MP, Moharir VM, Shin J et al. Comparison of endoscopic sinus surgery with and without image guidance. Am J Rhinol 2002; 16 : 193-197

[104] Ramakrishnan VR, Kingdom TT, Nayak JV et al. Nationwide incidence of major complications in endoscopic sinus surgery. Int Forum Allergy Rhinol 2012; 2: 34-39

[105] Krings JG, Kallogjeri D, Wineland A et al. Complications of primary and revision functional endoscopic sinus surgery for chronic rhinosinusitis. Laryngoscope 2014; 124: 838-845

[106] Javer AR, Genoway KA. Patient quality of life improvements with and without computer assistance in sinus surgery: outcomes study. J Otolaryngol 2006; 35: 373-379

[107] Galletti B, Gazia F, Freni F et al. Endoscopic sinus surgery with and without computer assisted navigation: A retrospective study. Auris Nasus Larynx 2018, doi:10.1016/j.anl.2018.11.004

[108] Eloy JA, Svider PF, D’Aguillo CM et al. Image-guidance in endoscopic sinus surgery: is it associated with decreased medicolegal liability? Int Forum Allergy Rhinol 2013; 3: 980-985 
[109] Ramakrishnan VR, Kingdom TT. Does Image-Guided Surgery Reduce Complications? Otolaryngol Clin North Am 2015; 48: 851-859

[110] Jankowski R, Rumeau C, Nguyen DT et al. Updating nasalisation: From concept to technique and results. Eur Ann Otorhinolaryngol Head Neck Dis 2018; 135: 327-334

[111] Alsharif S, Jonstam K, van Zele T et al. Endoscopic Sinus Surgery for Type-2 CRS wNP: An Endotype-Based Retrospective Study. Laryngoscope 2019, doi:10.1002/lary.27815

[112] Naidoo Y, Bassiouni A, Keen M et al. Risk factors and outcomes for primary, revision, and modified Lothrop (Draf III) frontal sinus surgery. Int Forum Allergy Rhinol 2013; 3: 412-417

[113] Naidoo Y, Bassiouni A, Keen M et al. Long-term outcomes for the endoscopic modified Lothrop/Draf III procedure: a 10-year review. Laryngoscope 2014; 124: 43-49

[114] Messerklinger W. On the drainage of the normal frontal sinus of man. Acta Otolaryngol 1967; 63: 176-181

[115] Stammberger H, Posawetz W. Functional endoscopic sinus surgery. Concept, indications and results of the Messerklinger technique. Eur Arch Otorhinolaryngol 1990; 247: 63-76

[116] Leung RM, Kern RC, Conley DB et al. Osteomeatal complex obstruction is not associated with adjacent sinus disease in chronic rhinosinusitis with polyps. Am J Rhinol Allergy 2011; 25: 401-403

[117] Chandra RK, Pearlman A, Conley DB et al. Significance of osteomeatal complex obstruction. J Otolaryngol Head Neck Surg 2010; 39 : $171-174$

[118] Wigand ME, Steiner W, Jaumann MP. Endonasal sinus surgery with endoscopical control: from radical operation to rehabilitation of the mucosa. Endoscopy 1978; 10: 255-260

[119] Goldstein GH, Kennedy DW. Long-term successes of various sinus surgeries: a comprehensive analysis. Curr Allergy Asthma Rep 2013; 13: $244-249$

[120] Larsen K, Tos M. A long-term follow-up study of nasal polyp patients after simple polypectomies. Eur Arch Otorhinolaryngol 1997; 254 (Suppl 1): S85-S88

[121] DeConde AS, Mace JC, Levy JM et al. Prevalence of polyp recurrence after endoscopic sinus surgery for chronic rhinosinusitis with nasal polyposis. Laryngoscope 2017; 127: 550-555

[122] Browne JP, Hopkins C, Slack R et al. Health-related quality of life after polypectomy with and without additional surgery. Laryngoscope 2006; 116: 297-302

[123] Hopkins C, Slack R, Lund V et al. Long-term outcomes from the English national comparative audit of surgery for nasal polyposis and chronic rhinosinusitis. Laryngoscope 2009; 119: 2459-2465

[124] Rudmik L, Smith KA, Kilty S. Endoscopic polypectomy in the clinic: a pilot cost-effectiveness analysis. Clin Otolaryngol 2016; 41: 110-117

[125] Kilty S], Lasso A, Mfuna-Endam L et al. Case-control study of endoscopic polypectomy in clinic (EPIC) versus endoscopic sinus surgery for chronic rhinosinusitis with polyps. Rhinology 2018; 56: 155-157

[126] Myller J, Dastidar P, Torkkeli T et al. Computed tomography findings after endoscopic sinus surgery with preserving or enlarging maxillary sinus ostium surgery. Rhinology 2011; 49: 438-444

[127] Luukkainen A, Myller J, Torkkeli T et al. Endoscopic sinus surgery with antrostomy has better early endoscopic recovery in comparison to the ostium-preserving technique. ISRN Otolaryngol 2012; 2012: 189383

[128] Valdes C], Bogado M, Samaha M. Causes of failure in endoscopic frontal sinus surgery in chronic rhinosinusitis patients. Int Forum Allergy Rhinol 2014; 4: 502-506

[129] Georgalas C, Videler W, Freling N et al. Global Osteitis Scoring Scale and chronic rhinosinusitis: a marker of revision surgery. Clin Otolaryngol 2010; 35: 455-461
[130] Nakayama T, Asaka D, Kuboki A et al. Impact of residual frontal recess cells on frontal sinusitis after endoscopic sinus surgery. Eur Arch Otorhinolaryngol 2018; 275: 1795-1801

[131] Bradley DT, Kountakis SE. The role of agger nasi air cells in patients requiring revision endoscopic frontal sinus surgery. Otolaryngol Head Neck Surg 2004; 131: 525-527

[132] Musy PY, Kountakis SE. Anatomic findings in patients undergoing revision endoscopic sinus surgery. Am J Otolaryngol 2004; 25: 418-422

[133] Otto KJ, DelGaudio JM. Operative findings in the frontal recess at time of revision surgery. Am J Otolaryngol 2010; 31: 175-180

[134] Abuzeid WM, Mace JC, Costa ML et al. Outcomes of chronic frontal sinusitis treated with ethmoidectomy: a prospective study. Int Forum Allergy Rhinol 2016; 6: 597-604

[135] Patel VS, Choby G, Shih LC et al. Equivalence in outcomes between Draf 2B vs Draf 3 frontal sinusotomy for refractory chronic frontal rhinosinusitis. Int Forum Allergy Rhinol 2018; 8: 25-31

[136] Havas TE, Lowinger DS. Comparison of functional endonasal sinus surgery with and without partial middle turbinate resection. Ann Otol Rhinol Laryngol 2000; 109: 634-640

[137] Marchioni D, Alicandri-Ciufelli M, Mattioli F et al. Middle turbinate preservation versus middle turbinate resection in endoscopic surgical treatment of nasal polyposis. Acta Otolaryngol 2008; 128 : 1019-1026

[138] Soler ZM, Hwang PH, Mace J et al. Outcomes after middle turbinate resection: revisiting a controversial topic. Laryngoscope 2010; 120 : 832-837

[139] Byun JY, Lee JY. Middle turbinate resection versus preservation in patients with chronic rhinosinusitis accompanying nasal polyposis: baseline disease burden and surgical outcomes between the groups. J Otolaryngol Head Neck Surg 2012; 41: 259-264

[140] Hudon MA, Wright ED, Fortin-Pellerin E et al. Resection versus preservation of the middle turbinate in surgery for chronic rhinosinusitis with nasal polyposis: a randomized controlled trial. J Otolaryngol Head Neck Surg 2018; 47: 67

[141] Akiyama K, Samukawa Y, Takahashi S et al. Clinical effects of submucosal middle turbinectomy for eosinophilic chronic rhinosinusitis. Auris Nasus Larynx 2018; 45: 765-771

[142] Scangas GA, Remenschneider AK, Bleier BS et al. Does the Timing of Middle Turbinate Resection Influence Quality-of-Life Outcomes for Patients with Chronic Rhinosinusitis? Otolaryngol Head Neck Surg 2017; 157: 874-879

[143] Tan NC, Goggin R, Psaltis AJ et al. Partial resection of the middle turbinate during endoscopic sinus surgery for chronic rhinosinusitis does not lead to an increased risk of empty nose syndrome: a cohort study of a tertiary practice. Int Forum Allergy Rhinol 2018, doi:10.1002/alr.22127

[144] King JM, Caldarelli DD, Pigato JB. A review of revision functional endoscopic sinus surgery. Laryngoscope 1994; 104: 404-408

[145] Videler WJ, Wreesmann VB, van der Meulen FW et al. Repetitive endoscopic sinus surgery failure: a role for radical surgery? Otolaryngol Head Neck Surg 2006; 134: 586-591

[146] Eloy JA, Marchiano E, Vazquez A. Extended Endoscopic and Open Sinus Surgery for Refractory Chronic Rhinosinusitis. Otolaryngol Clin North Am 2017; 50: 165-182

[147] Thulasidas P, Vaidyanathan V. Role of modified endoscopic medial maxillectomy in persistent chronic maxillary sinusitis. Int Arch Otorhinolaryngol 2014; 18: 159-164

[148] Costa ML, Psaltis AJ, Nayak JV et al. Long-term outcomes of endoscopic maxillary mega-antrostomy for refractory chronic maxillary sinusitis. Int Forum Allergy Rhinol 2015; 5: 60-65 
[149] DeConde AS, Suh JD, Mace JC et al. Outcomes of complete vs targeted approaches to endoscopic sinus surgery. Int Forum Allergy Rhinol 2015; 5: 691-700

[150] Chen FH, Deng J, Hong HY et al. Extensive versus functional endoscopic sinus surgery for chronic rhinosinusitis with nasal polyps and asthma: A 1-year study. Am J Rhinol Allergy 2016; 30: 143-148

[151] Morrissey DK, Bassiouni A, Psaltis AJ et al. Outcomes of modified endoscopic Lothrop in aspirin-exacerbated respiratory disease with nasal polyposis. Int Forum Allergy Rhinol 2016; 6: 820-825

[152] Southwood JE, Loehrl TA, Poetker DM. Advances in Surgery: Extended Procedures for Sinonasal Polyp Disease. Adv Otorhinolaryngol 2016; 79: $148-157$

[153] Keerl R, Weber R, Drees $G$ et al. Individual learning curves with reference to endonasal micro-endoscopic pan-sinus operation. Laryngorhinootologie 1996; 75: 338-343

[154] Laeeq K, Lin SY, Varela DA et al. Achievement of competency in endoscopic sinus surgery of otolaryngology residents. Laryngoscope 2013; 123: 2932-2934

[155] Tang J, Tan S, Fang Q et al. Investigate of the learning curve of cochlear implantation. Zhonghua Er Bi Yan Hou Tou Jing Wai Ke Za Zhi 2014; 49: 649-653

[156] Liu CY, Yu EC, Lin SH et al. Learning curve of septomeatoplasty. Auris Nasus Larynx 2009; 36: 661-664

[157] Tseng CC, Lai MT, Wu CC et al. Learning curve for endoscopic tympanoplasty: Initial experience of 221 procedures. J Chin Med Assoc 2017; 80: 508-514

[158] Dogan S, Bayraktar C. Endoscopic tympanoplasty: learning curve for a surgeon already trained in microscopic tympanoplasty. Eur Arch Otorhinolaryngol 2017; 274: 1853-1858

[159] Awad Z, Taghi A, Sethukumar P et al. Construct validity of the ovine model in endoscopic sinus surgery training. Laryngoscope 2015; 125 : 539-543

[160] Montague ML, Kishore A, McGarry GW. Audit-derived guidelines for training in endoscopic sinonasal surgery (ESS) - protecting patients during the learning curve. Clin Otolaryngol Allied Sci 2003; 28: 411-416

[161] Ramadan HH, Allen GC. Complications of endoscopic sinus surgery in a residency training program. Laryngoscope 1995; 105: 376-379

[162] Phillips JS, Vowler SL, Salam MA. Is training in endoscopic sinus surgery detrimental to patient outcome? J Surg Educ 2007; 64: 278-281

[163] Braun T, Betz CS, Stelter $\mathrm{K}$ et al. FESS and surgical training: what are the problems?. Laryngorhinootologie 2011; 90: 10-14

[164] Bakker NH, Fokkens W], Grimbergen CA. Investigation of training needs for functional endoscopic sinus surgery (FESS). Rhinology 2005; 43: 104-108

[165] Zuckerman JD, Wise SK, Rogers GA et al. The utility of cadaver dissection in endoscopic sinus surgery training courses. Am J Rhinol Allergy 2009; 23: 218-224

[166] Palter VN, Grantcharov TP. Development and validation of a comprehensive curriculum to teach an advanced minimally invasive procedure: a randomized controlled trial. Ann Surg 2012; 256: 25-32

[167] Mladina R, Skitarelic N, Cingi C et al. The Validity of Training Endoscopic Sinus and Skull Base Surgery Techniques on the Experimental Head Model. J Craniofac Surg 2018; 29: 498-501

[168] Fried MP, Sadoughi B, Gibber MJ et al. From virtual reality to the operating room: the endoscopic sinus surgery simulator experiment. Otolaryngol Head Neck Surg 2010; 142: 202-207

[169] Varshney R, Frenkiel S, Nguyen LH et al. The McGill simulator for endoscopic sinus surgery (MSESS): a validation study. J Otolaryngol Head Neck Surg 2014; 43: 40
[170] Harbison RA, Johnson KE, Miller C et al. Face, content, and construct validation of a low-cost, non-biologic, sinus surgery task trainer and knowledge-based curriculum. Int Forum Allergy Rhinol 2017; 7: 405-413

[171] Tolsdorff B, Pommert A, Hohne KH et al. Virtual reality: a new paranasal sinus surgery simulator. Laryngoscope 2010; 120: 420-426

[172] Briner HR, Simmen D, Jones $\mathrm{N}$ et al. Evaluation of an anatomic model of the paranasal sinuses for endonasal surgical training. Rhinology 2007; 45: 20-23

[173] Nogueira JF, Stamm AC, Lyra M et al. Building a real endoscopic sinus and skull-base surgery simulator. Otolaryngol Head Neck Surg 2008; 139: $727-728$

[174] Malekzadeh S, Pfisterer MJ, Wilson B et al. A novel low-cost sinus surgery task trainer. Otolaryngol Head Neck Surg 2011; 145: 530-533

[175] Uribe JI, Ralph WM Jr., Glaser AY et al. Learning curves, acquisition, and retention of skills trained with the endoscopic sinus surgery simulator. Am J Rhinol 2004; 18: 87-92

[176] Edmond CV Jr.. Impact of the endoscopic sinus surgical simulator on operating room performance. Laryngoscope 2002; 112: 1148-1158

[177] Clifton N, Klingmann C, Khalil H. Teaching Otolaryngology skills through simulation. Eur Arch Otorhinolaryngol 2011; 268: 949-953

[178] Hosemann W, Draf C. Danger points, complications and medico-legal aspects in endoscopic sinus surgery. GMS Curr Top Otorhinolaryngol Head Neck Surg 2013; 12: Doc06

[179] Wanzel KR, Ward M, Reznick RK. Teaching the surgical craft: From selection to certification. Curr Probl Surg 2002; 39: 573-659

[180] Beard JD, Choksy S, Khan S et al. Assessment of operative competence during carotid endarterectomy. Br J Surg 2007; 94: 726-730

[181] Goff B, Mandel L, Lentz G et al. Assessment of resident surgical skills: is testing feasible? Am J Obstet Gynecol 2005; 192: 1331-1338 discussion 1338-1340

[182] Anderson DD, Long S, Thomas GW et al. Objective Structured Assessments of Technical Skills (OSATS) Does Not Assess the Quality of the Surgical Result Effectively. Clin Orthop Relat Res 2016; 474: 874-881

[183] Syme-Grant ], White PS, McAleer JP. Measuring competence in endoscopic sinus surgery. Surgeon 2008; 6: 37-44

[184] Lin SY, Laeeq K, Ishii M et al. Development and pilot-testing of a feasible, reliable, and valid operative competency assessment tool for endoscopic sinus surgery. Am J Rhinol Allergy 2009; 23: 354-359

[185] Laeeq K, Waseem R, Weatherly RA et al. In-training assessment and predictors of competency in endoscopic sinus surgery. Laryngoscope 2010; 120: 2540-2545

[186] Marglani O, Alherabi A, Al-Andejani T et al. Development of a tool for Global Rating of Endoscopic Surgical Skills (GRESS) for assessment of otolaryngology residents. B-ENT 2012; 8: 191-195

[187] Chowdhury MM, Dagash H, Pierro A. A systematic review of the impact of volume of surgery and specialization on patient outcome. Br J Surg 2007; 94: 145-161

[188] Eggli Y, Halfon P, Meylan D et al. Surgical safety and hospital volume across a wide range of interventions. Med Care 2010; 48: 962-971

[189] Birkmeyer JD, Siewers AE, Finlayson EV et al. Hospital volume and surgical mortality in the United States. N Engl J Med 2002; 346: 1128-1137

[190] Duclos A, Peix JL, Colin C et al. Influence of experience on performance of individual surgeons in thyroid surgery: prospective cross sectional multicentre study. BM] 2012; 344: d8041

[191] Choudhry NK, Fletcher RH, Soumerai SB. Systematic review: the relationship between clinical experience and quality of health care. Ann Intern Med 2005; 142: 260-273 
[192] Southern WN, Bellin EY, Arnsten JH. Longer lengths of stay and higher risk of mortality among inpatients of physicians with more years in practice. Am J Med 2011; 124: 868-874

[193] Hannan EL, Popp AJ, Feustel P et al. Association of surgical specialty and processes of care with patient outcomes for carotid endarterectomy. Stroke 2001; 32: 2890-2897

[194] Kennedy GT, McMillan MT, Sprys MH et al. The influence of fellowship training on the practice of pancreatoduodenectomy. HPB (Oxford) 2016; 18: 965-978

[195] Sahni NR, Dalton M, Cutler DM et al. Surgeon specialization and operative mortality in United States: retrospective analysis. BM] 2016; 354: i3571

[196] Sainsbury R, Haward B, Rider L et al. Influence of clinician workload and patterns of treatment on survival from breast cancer. Lancet 1995; 345: 1265-1270

[197] Kockerling F, Bittner R, Kraft B et al. Does surgeon volume matter in the outcome of endoscopic inguinal hernia repair? Surg Endosc 2017; 31: 573-585

[198] Smith TL, Mace JC, Rudmik L et al. Comparing surgeon outcomes in endoscopic sinus surgery for chronic rhinosinusitis. Laryngoscope 2017; 127: 14-21

[199] Rudmik L, Xu Y, Alt JA et al. Evaluating Surgeon-Specific Performance for Endoscopic Sinus Surgery. JAMA Otolaryngol Head Neck Surg 2017; 143: 891-898

[200] Schlosser RJ, Storck K, Smith TL et al. Impact of postoperative endoscopy upon clinical outcomes after endoscopic sinus surgery. Int Forum Allergy Rhinol 2016; 6: 115-123

[201] Snidvongs K, Dalgorf D, Kalish L et al. Modified Lund Mackay Postoperative Endoscopy Score for defining inflammatory burden in chronic rhinosinusitis. Rhinology 2014; 52: 53-59

[202] Stewart MG, Johnson RF. Chronic sinusitis: symptoms versus CT scan findings. Curr Opin Otolaryngol Head Neck Surg 2004; 12: 27-29

[203] Ten Dam E, Feijen RA, van den Berge MJC et al. Development of the Endoscopic Endonasal Sinus and Skull Base Surgery Questionnaire. Int Forum Allergy Rhinol 2017; 7: 1076-1084

[204] Cella DF, Bonomi AE. Measuring quality of life: 1995 update. Oncology (Williston Park) 1995; 9: 47-60

[205] Rudmik L, Hopkins C, Peters A et al. Patient-reported outcome measures for adult chronic rhinosinusitis: A systematic review and quality assessment. J Allergy Clin Immunol 2015; 136: 1532-1540 e1532

[206] Baumann I. Validated instruments to measure quality of life in patients with chronic rhinosinusitis. HNO 2009; 57: 873-881

[207] Shen B, Liu LT, Liu D et al. Comparison of different surgical approaches of functional endoscopic sinus surgery on patients with chronic rhinosinusitis. Int J Clin Exp Med 2014; 7: 1585-1591

[208] Djukic V, Dudvarski Z, Arsovic N et al. Clinical outcomes and quality of life in patients with nasal polyposis after functional endoscopic sinus surgery. Eur Arch Otorhinolaryngol 2015; 272: 83-89

[209] Katotomichelakis M, Simopoulos E, Tripsianis G et al. Predictors of quality of life outcomes in chronic rhinosinusitis after sinus surgery. Eur Arch Otorhinolaryngol 2014; 271: 733-741

[210] Smith TL, Litvack JR, Hwang PH et al. Determinants of outcomes of sinus surgery: a multi-institutional prospective cohort study. Otolaryngol Head Neck Surg 2010; 142: 55-63

[211] Croy I, Hummel T, Pade A et al. Quality of life following nasal surgery. Laryngoscope 2010; 120: 826-831

[212] Lehmann AE, Scangas GA, Sethi RKV et al. Impact of Age on Sinus Surgery Outcomes. Laryngoscope 2018; 128: 2681-2687
[213] Remenschneider AK, D'Amico L, Litvack JR et al. Long-Term Outcomes in Sinus Surgery: A New Tool for Measuring Health-Related Quality of Life. Otolaryngol Head Neck Surg 2014; 151: 164-170

[214] Rudmik L, Smith TL. Quality of life in patients with chronic rhinosinusitis. Curr Allergy Asthma Rep 2011; 11: 247-252

[215] Soler ZM, Smith TL, Alt JA et al. Olfactory-specific quality of life outcomes after endoscopic sinus surgery. Int Forum Allergy Rhinol 2016; 6: 407-413

[216] Mattos JL, Schlosser RJ, Mace JC et al. Establishing the minimal clinically important difference for the Questionnaire of Olfactory Disorders. Int Forum Allergy Rhinol 2018; 8: 1041-1046

[217] Mattos JL, Schlosser RJ, DeConde AS et al. Factor analysis of the questionnaire of olfactory disorders in patients with chronic rhinosinusitis. Int Forum Allergy Rhinol 2018; 8: 777-782

[218] Katotomichelakis M, Simopoulos E, Tripsianis G et al. Improvement of olfactory function for quality of life recovery. Laryngoscope 2013; 123: E10-E16

[219] Banglawala SM, Schlosser R], Morella K et al. Qualitative development of the sinus control test: a survey evaluating sinus symptom control. Int Forum Allergy Rhinol 2016; 6: 491-499

[220] Smith TL, Kern R, Palmer JN et al. Medical therapy vs. surgery for chronic rhinosinusitis: a prospective, multi-institutional study with 1-year follow-up. Int Forum Allergy Rhinol 2013; 3: 4-9

[221] Smith TL, Kern RC, Palmer JN et al. Medical therapy vs surgery for chronic rhinosinusitis: a prospective, multi-institutional study. Int Forum Allergy Rhinol 2011; 1: 235-241

[222] Soler ZM, Smith TL. Quality-of-life outcomes after endoscopic sinus surgery: how long is long enough? Otolaryngol Head Neck Surg 2010; 143: 621-625

[223] Clinger JD, Mace JC, Smith TL. Quality-of-life outcomes following multiple revision endoscopic sinus surgery. Int Forum Allergy Rhinol 2012; 2: 444-452

[224] Browne JP, Hopkins C, Slack R et al. The Sino-Nasal Outcome Test (SNOT): can we make it more clinically meaningful? Otolaryngol Head Neck Surg 2007; 136: 736-741

[225] Pynnonen MA, Kim HM, Terrell JE. Validation of the Sino-Nasal Outcome Test 20 (SNOT-20) domains in nonsurgical patients. Am J Rhinol Allergy 2009; 23: 40-45

[226] DeConde AS, Bodner TE, Mace JC et al. Response shift in quality of life after endoscopic sinus surgery for chronic rhinosinusitis. JAMA Otolaryngol Head Neck Surg 2014; 140: 712-719

[227] Feng AL, Wesely NC, Hoehle LP et al. A validated model for the 22-item Sino-Nasal Outcome Test subdomain structure in chronic rhinosinusitis. Int Forum Allergy Rhinol 2017; 7: 1140-1148

[228] Quintanilla-Dieck L, Litvack JR, Mace JC et al. Comparison of disease-specific quality-of-life instruments in the assessment of chronic rhinosinusitis. Int Forum Allergy Rhinol 2012; 2: 437-443

[229] Hopkins C, Gillett S, Slack R et al. Psychometric validity of the 22-item Sinonasal Outcome Test. Clin Otolaryngol 2009; 34: 447-454

[230] Chowdhury NI, Mace JC, Bodner TE et al. Investigating the minimal clinically important difference for SNOT-22 symptom domains in surgically managed chronic rhinosinusitis. Int Forum Allergy Rhinol 2017; 7: 1149-1155

[231] Levy JM, Mace JC, Rudmik L et al. Low 22-item sinonasal outcome test scores in chronic rhinosinusitis: Why do patients seek treatment? Laryngoscope 2017; 127: 22-28

[232] Singla G, Singh M, Singh A et al. Is sino-nasal outcome test-22 reliable for guiding chronic rhinosinusitis patients for endoscopic sinus surgery? Niger J Clin Pract 2018; 21: 1228-1233 
[233] Beswick DM, Mace JC, Rudmik L et al. Socioeconomic factors impact quality of life outcomes and olfactory measures in chronic rhinosinusitis. Int Forum Allergy Rhinol 2019; 9: 231-239

[234] Erskine S, Hopkins C, Kumar $\mathrm{N}$ et al. A cross sectional analysis of a case-control study about quality of life in CRS in the UK; a comparison between CRS subtypes. Rhinology 2016; 54: 311-315

[235] Sahlstrand-Johnson P, Ohlsson B, Von Buchwald C et al. A multicentre study on quality of life and absenteeism in patients with CRS referred for endoscopic surgery. Rhinology 2011; 49: 420-428

[236] Lal D, Rounds AB, Divekar R. Gender-specific differences in chronic rhinosinusitis patients electing endoscopic sinus surgery. Int Forum Allergy Rhinol 2016; 6: 278-286

[237] Lal D, Golisch KB, Elwell ZA et al. Gender-specific analysis of outcomes from endoscopic sinus surgery for chronic rhinosinusitis. Int Forum Allergy Rhinol 2016; 6: 896-905

[238] Erskine SE, Hopkins C, Clark A et al. SNOT-22 in a control population. Clin Otolaryngol 2017; 42: 81-85

[239] Mascarenhas JG, da Fonseca VM, Chen VG et al. Long-term outcomes of endoscopic sinus surgery for chronic rhinosinusitis with and without nasal polyps. Braz J Otorhinolaryngol 2013; 79: 306-311

[240] Kosugi EM, Chen VG, Fonseca VM et al. Translation, cross-cultural adaptation and validation of SinoNasal Outcome Test (SNOT): 22 to Brazilian Portuguese. Braz J Otorhinolaryngol 2011; 77: 663-669

[241] Zhang Z, Adappa ND, Doghramji L] et al. Quality of life improvement from sinus surgery in chronic rhinosinusitis patients with asthma and nasal polyps. Int Forum Allergy Rhinol 2014; 4: 885-892

[242] DeConde AS, Mace JC, Alt JA et al. Longitudinal improvement and stability of the SNOT-22 survey in the evaluation of surgical management for chronic rhinosinusitis. Int Forum Allergy Rhinol 2015; 5: 233-239

[243] Savastano V, Bertin S, Vittori T et al. Evaluation of chronic rhinosinusitis management using the SNOT-22 in adult cystic fibrosis patients. Eur Rev Med Pharmacol Sci 2014; 18: 1985-1989

[244] Alt JA, Smith TL, Schlosser RJ et al. Sleep and quality of life improvements after endoscopic sinus surgery in patients with chronic rhinosinusitis. Int Forum Allergy Rhinol 2014; 4: 693-701

[245] El Rassi E, Mace JC, Steele TO et al. Improvements in sleep-related symptoms after endoscopic sinus surgery in patients with chronic rhinosinusitis. Int Forum Allergy Rhinol 2016; 6: 414-422

[246] Levy JM, Mace JC, DeConde AS et al. Improvements in psychological dysfunction after endoscopic sinus surgery for patients with chronic rhinosinusitis. Int Forum Allergy Rhinol 2016; 6: 906-913

[247] Alt JA, Smith TL, Mace JC et al. Sleep quality and disease severity in patients with chronic rhinosinusitis. Laryngoscope 2013; 123 : 2364-2370

[248] Alt JA, Ramakrishnan VR, Platt MP et al. Sleep quality outcomes after medical and surgical management of chronic rhinosinusitis. Int Forum Allergy Rhinol 2017; 7: 113-118

[249] Rotenberg BW, Pang KP. The impact of sinus surgery on sleep outcomes. Int Forum Allergy Rhinol 2015; 5: 329-332

[250] Alakarppa AI, Koskenkorva T], Koivunen PT et al. Quality of life before and after sinonasal surgery: a population-based matched cohort study. Eur Arch Otorhinolaryngol 2017; 274: 795-802

[251] Rudmik L, Soler ZM, Hopkins C. Using postoperative SNOT-22 to help predict the probability of revision sinus surgery. Rhinology 2016; 54 : $111-116$

[252] Campbell AP, Hoehle LP, Phillips KM et al. Symptom control in chronic rhinosinusitis is an independent predictor of productivity loss. Eur Ann Otorhinolaryngol Head Neck Dis 2018; 135: 237-241

[253] Campbell AP, Phillips KM, Hoehle LP et al. Depression symptoms and lost productivity in chronic rhinosinusitis. Ann Allergy Asthma Immunol 2017; 118: 286-289
[254] Rudmik L, Smith TL, Mace JC et al. Productivity costs decrease after endoscopic sinus surgery for refractory chronic rhinosinusitis. Laryngoscope 2016; 126: 570-574

[255] Rudmik L, Soler ZM, Smith TL et al. Effect of Continued Medical Therapy on Productivity Costs for Refractory Chronic Rhinosinusitis. JAMA Otolaryngol Head Neck Surg 2015; 141: 969-973

[256] Lund V], Kennedy DW. Staging for rhinosinusitis. Otolaryngol Head Neck Surg 1997; 117: S35-S40

[257] Psaltis AJ, Li G, Vaezeafshar R et al. Modification of the Lund-Kennedy endoscopic scoring system improves its reliability and correlation with patient-reported outcome measures. Laryngoscope 2014; 124 : 2216-2223

[258] Litvack JR, Griest S, James KE et al. Endoscopic and quality-of-life outcomes after revision endoscopic sinus surgery. Laryngoscope 2007; 117: 2233-2238

[259] Lee JY, Lee SW, Lee JD. Comparison of the surgical outcome between primary and revision endoscopic sinus surgery for chronic rhinosinusitis with nasal polyposis. Am J Otolaryngol 2008; 29: 379-384

[260] McMains KC, Kountakis SE. Revision functional endoscopic sinus surgery: objective and subjective surgical outcomes. Am J Rhinol 2005; 19: 344-347

[261] Lind H, Joergensen G, Lange B et al. Efficacy of ESS in chronic rhinosinusitis with and without nasal polyposis: a Danish cohort study. Eur Arch Otorhinolaryngol 2016; 273: 911-919

[262] Wright ED, Agrawal S. Impact of perioperative systemic steroids on surgical outcomes in patients with chronic rhinosinusitis with polyposis: evaluation with the novel Perioperative Sinus Endoscopy (POSE) scoring system. Laryngoscope 2007; 117: 1-28

[263] Durr ML, Pletcher SD, Goldberg AN et al. A novel sinonasal endoscopy scoring system: the discharge, inflammation, and polyps/edema (DIP) score. Int Forum Allergy Rhinol 2013; 3: 66-72

[264] Philpott C, Hopkins C, Erskine S et al. The burden of revision sinonasal surgery in the UK-data from the Chronic Rhinosinusitis Epidemiology Study (CRES): a cross-sectional study. BMJ Open 2015; 5: e006680

[265] Smith KA, Orlandi RR, Oakley G et al. Long-term revision rates for endoscopic sinus surgery. Int Forum Allergy Rhinol 2019; 9: 402-408

[266] Stein NR, Jafari A, DeConde AS. Revision rates and time to revision following endoscopic sinus surgery: A large database analysis. Laryngoscope 2018; 128: 31-36

[267] Senior BA, Kennedy DW, Tanabodee J et al. Long-term results of functional endoscopic sinus surgery. Laryngoscope 1998; 108: 151-157

[268] Penttila M, Rautiainen M, Pukander ] et al. Functional vs. radical maxillary surgery. Failures after functional endoscopic sinus surgery. Acta Otolaryngol Suppl 1997; 529: 173-176

[269] Mendelsohn D, Jeremic G, Wright ED et al. Revision rates afte endoscopic sinus surgery: a recurrence analysis. Ann Otol Rhinol Laryngol 2011; 120: 162-166

[270] Wu AW, Ting JY, Platt MP et al. Factors affecting time to revision sinus surgery for nasal polyps: a 25-year experience. Laryngoscope 2014; 124: 29-33

[271] Hopkins C, Browne JP, Slack R et al. Complications of surgery for nasa polyposis and chronic rhinosinusitis: the results of a national audit in England and Wales. Laryngoscope 2006; 116: 1494-1499

[272] Dalziel K, Stein K, Round A et al. Endoscopic sinus surgery for the excision of nasal polyps: A systematic review of safety and effectiveness. Am J Rhinol 2006; 20: 506-519

[273] Chaaban MR, Rana N, Baillargeon J et al. Outcomes and Complications of Balloon and Conventional Functional Endoscopic Sinus Surgery. Am J Rhinol Allergy 2018; 32: 388-396 
[274] Suzuki S, Yasunaga H, Matsui $\mathrm{H}$ et al. Complication rates after functional endoscopic sinus surgery: analysis of 50,734 Japanese patients. Laryngoscope 2015; 125: 1785-1791

[275] Asaka D, Nakayama T, Hama T et al. Risk factors for complications of endoscopic sinus surgery for chronic rhinosinusitis. Am J Rhinol Allergy 2012; 26: 61-64

[276] Hummel T, Kobal G, Gudziol H et al. Normative data for the „Sniffin’ Sticks" including tests of odor identification, odor discrimination, and olfactory thresholds: an upgrade based on a group of more than 3,000 subjects. Eur Arch Otorhinolaryngol 2007; 264: 237-243

[277] Doty RL, Shaman P, Dann M. Development of the University of Pennsylvania Smell Identification Test: a standardized microencapsulated test of olfactory function. Physiol Behav 1984; 32: 489-502

[278] Doty RL, Marcus A, Lee WW. Development of the 12-item CrossCultural Smell Identification Test (CC-SIT). Laryngoscope 1996; 106: 353-356

[279] Gudziol V, Lotsch ], Hahner A et al. Clinical significance of results from olfactory testing. Laryngoscope 2006; 116: 1858-1863

[280] Haxel BR, Boessert P, Weyer-Elberich V et al. Course of olfaction after sinus surgery for chronic rhinosinusitis. Laryngoscope Investig Otolaryngol 2017; 2: 269-275

[281] Pade J, Hummel T. Olfactory function following nasal surgery. Laryngoscope 2008; 118: 1260-1264

[282] Hsu CY, Wang YP, Shen PH et al. Objective olfactory outcomes after revision endoscopic sinus surgery. Am J Rhinol Allergy 2013; 27: e96-e100

[283] Klossek JM, Peloquin L, Friedman WH et al. Diffuse nasal polyposis: postoperative long-term results after endoscopic sinus surgery and frontal irrigation. Otolaryngol Head Neck Surg 1997; 117: 355-361

[284] Nguyen DT, Gauchotte G, Nguyen-Thi PL et al. Does surgery of the olfactory clefts modify the sense of smell? Am J Rhinol Allergy 2013; 27: $317-321$

[285] Kuperan AB, Lieberman SM, Jourdy DN et al. The effect of endoscopic olfactory cleft polyp removal on olfaction. Am J Rhinol Allergy 2015; 29: 309-313

[286] Rudmik L, Smith TL. Olfactory improvement after endoscopic sinus surgery. Curr Opin Otolaryngol Head Neck Surg 2012; 20: 29-32

[287] Litvack JR, Mace J, Smith TL. Does olfactory function improve after endoscopic sinus surgery? Otolaryngol Head Neck Surg 2009; 140: 312-319

[288] Kohli P, Naik AN, Farhood Z et al. Olfactory Outcomes after Endoscopic Sinus Surgery for Chronic Rhinosinusitis: A Meta-analysis. Otolaryngol Head Neck Surg 2016; 155: 936-948

[289] Andrews PJ, Poirrier AL, Lund V] et al. Outcomes in endoscopic sinus surgery: olfaction, nose scale and quality of life in a prospective cohort study. Clin Otolaryngol 2016; 41: 798-803

[290] AlBader A, Levine CG, Casiano RR. Does endoscopic sinus surgery improve olfaction in nasal polyposis? Laryngoscope 2017; 127 : 2203-2204

[291] Williamson PR, Altman DG, Blazeby JM et al. Developing core outcome sets for clinical trials: issues to consider. Trials 2012; 13: 132

[292] Kirkham J], Davis K, Altman DG et al. Core Outcome Set-STAndards for Development: The COS-STAD recommendations. PLoS Med 2017; 14: e 1002447

[293] Hopkins C, Hettige R, Soni-Jaiswal A et al. CHronic Rhinosinusitis Outcome MEasures (CHROME), developing a core outcome set for trials of interventions in chronic rhinosinusitis. Rhinology 2018; 56: 22-32

[294] Rimmer J, Fokkens W, Chong LY et al. Surgical versus medical interventions for chronic rhinosinusitis with nasal polyps. Cochrane Database Syst Rev 2014; doi:10.1002/14651858.CD006991.pub2 CD006991
[295] Khalil HS, Nunez DA. Functional endoscopic sinus surgery for chronic rhinosinusitis. Cochrane Database Syst Rev 2006; doi:10.1002/14651858.CD004458.pub2 CD004458

[296] Dessouky O, Hopkins C. Surgical versus medical interventions in CRS and nasal polyps: comparative evidence between medical and surgical efficacy. Curr Allergy Asthma Rep 2015; 15: 66

[297] Rix I, Hakansson K, Larsen CG et al. Management of chronic rhinosinusitis with nasal polyps and coexisting asthma: A systematic review. Am J Rhinol Allergy 2015; 29: 193-201

[298] Wente MN, Seiler CM, Uhl W et al. Perspectives of evidence-based surgery. Dig Surg 2003; 20: 263-269

[299] Smith KA, Smith TL, Mace JC et al. Endoscopic sinus surgery compared to continued medical therapy for patients with refractory chronic rhinosinusitis. Int Forum Allergy Rhinol 2014; 4: 823-827

[300] Smith KA, Rudmik L. Impact of continued medical therapy in patients with refractory chronic rhinosinusitis. Int Forum Allergy Rhinol 2014; 4: $34-38$

[301] Steele TO, Rudmik L, Mace JC et al. Patient-centered decision making: the role of the baseline SNOT-22 in predicting outcomes for medical management of chronic rhinosinusitis. Int Forum Allergy Rhinol 2016; 6: 590-596

[302] Luk L], Steele TO, Mace JC et al. Health utility outcomes in patients undergoing medical management for chronic rhinosinusitis: a prospective multiinstitutional study. Int Forum Allergy Rhinol 2015; 5: 1018-1027

[303] De Greve G, Hellings PW, Fokkens W] et al. Endotype-driven treatment in chronic upper airway diseases. Clin Transl Allergy 2017; 7: 22

[304] Scangas GA, Remenschneider AK, Su BM et al. Cost utility analysis of endoscopic sinus surgery for chronic rhinosinusitis with and without nasal polyposis. Laryngoscope 2017; 127: 29-37

[305] Scangas GA, Remenschneider AK, Su BM et al. The impact of asthma on the cost effectiveness of surgery for chronic rhinosinusitis with nasal polyps. Int Forum Allergy Rhinol 2017; 7: 1035-1044

[306] Promsopa C, Kansara S, Citardi M] et al. Prevalence of confirmed asthma varies in chronic rhinosinusitis subtypes. Int Forum Allergy Rhinol 2016; 6: 373-377

[307] Bhattacharyya N, Grebner ], Martinson NG. Recurrent acute rhinosinusitis: epidemiology and health care cost burden. Otolaryngol Head Neck Surg 2012; 146: 307-312

[308] Costa ML, Psaltis A], Nayak JV et al. Medical therapy vs surgery for recurrent acute rhinosinusitis. Int Forum Allergy Rhinol 2015; 5: 667-673

[309] Poetker DM, Litvack JR, Mace JC et al. Recurrent acute rhinosinusitis: presentation and outcomes of sinus surgery. Am J Rhinol 2008; 22: 329-333

[310] Alkire BC, Bhattacharyya N. An assessment of sinonasal anatomic variants potentially associated with recurrent acute rhinosinusitis. Laryngoscope 2010; 120: 631-634

[311] Kaper NM, Breukel L, Venekamp RP et al. Absence of evidence for enhanced benefit of antibiotic therapy on recurrent acute rhinosinusitis episodes: a systematic review of the evidence base. Otolaryngol Head Neck Surg 2013; 149: 664-667

[312] Qvarnberg Y, Kantola O, Salo J et al. Influence of topical steroid treatment on maxillary sinusitis. Rhinology 1992; 30: 103-112

[313] van Loon JW, van Harn RP, Venekamp RP et al. Limited evidence for effects of intranasal corticosteroids on symptom relief for recurrent acute rhinosinusitis. Otolaryngol Head Neck Surg 2013; 149: 668-673

[314] Rudmik L, Beswick DM, Alt JA et al. Appropriateness Criteria for Surgery in the Management of Adult Recurrent Acute Rhinosinusitis. Laryngoscope 2019; 129: 37-44 
[315] Bhattacharyya N. Surgical treatment of chronic recurrent rhinosinusitis: a preliminary report. Laryngoscope 2006; 116: 1805-1808

[316] Michalowski A, Kacker A. Is sinus surgery indicated for recurrent acute rhinosinusitis? Laryngoscope 2017; 127: 1255-1256

[317] Steele TO, Detwiller KY, Mace JC et al. Productivity outcomes following endoscopic sinus surgery for recurrent acute rhinosinusitis. Laryngoscope 2016; 126: 1046-1053

[318] Steele TO, Mace JC, Dedhia R et al. Health utility values for patients with recurrent acute rhinosinusitis undergoing endoscopic sinus surgery: a nested case control study. Int Forum Allergy Rhinol 2016; 6: $1182-1187$

[319] Sikand A, Ehmer DR Jr., Stolovitzky JP et al. In-office balloon sinus dilation versus medical therapy for recurrent acute rhinosinusitis: a randomized, placebo-controlled study. Int Forum Allergy Rhinol 2019; 9: 140-148

[320] Bhandarkar ND, Mace JC, Smith TL. Endoscopic sinus surgery reduces antibiotic utilization in rhinosinusitis. Int Forum Allergy Rhinol 2011; 1: $18-22$
[321] Rizzi CF, Ferraz MB, Poyares D et al. Quality-adjusted life-years gain and health status in patients with OSAS after one year of continuous positive airway pressure use. Sleep 2014; 37: 1963-1968

[322] Feeny D, Wu L, Eng K. Comparing short form 6D, standard gamble, and Health Utilities Index Mark 2 and Mark 3 utility scores: results from total hip arthroplasty patients. Qual Life Res 2004; 13 : 1659-1670

[323] Bhattacharyya N, Lee KH. Chronic recurrent rhinosinusitis: disease severity and clinical characterization. Laryngoscope 2005; 115 : 306-310

[324] Sohn HG, Park SJ, Ryu IS et al. Comparison of Clinical Presentation and Surgical Outcomes Between Recurrent Acute Rhinosinusitis and Chronic Rhinosinusitis. Ann Otol Rhinol Laryngol 2018; 127: 763-769

[325] Leung R, Kern RC, Conley DB et al. Establishing a threshold for surgery in recurrent acute rhinosinusitis: a productivity-based analysis. Otolaryngol Head Neck Surg 2012; 146: 829-833

[326] Leung R, Almassian S, Kern R et al. Patient level decision making in recurrent acute rhinosinusitis: a cost-benefit threshold for surgery. Laryngoscope 2013; 123: 11-16 